\title{
Review \\ Development and Functionalization of Visible-Light-Driven Water-Splitting Photocatalysts
}

\author{
Tokuhisa Kawawaki ${ }^{1,2,3,+} \mathbb{1}$, Masanobu Kawachi ${ }^{1,+}{ }^{\dagger}$, Daichi Yazaki ${ }^{1}$, Yuki Akinaga ${ }^{1}$, Daisuke Hirayama ${ }^{1}$ \\ and Yuichi Negishi $1,2,3, *$ (D)
}

1 Department of Applied Chemistry, Faculty of Science, Tokyo University of Science, Kagurazaka, Shinjuku-ku, Tokyo 162-8601, Japan; kawawaki@rs.tus.ac.jp (T.K.); iwish117@outlook.jp (M.K.); dunkshot1997.tus.ok@gmail.com (D.Y.); yuki.tennis.k.1214@gmail.com (Y.A.); d24.bsk5@gmail.com (D.H.)

2 Research Institute for Science \& Technology, Tokyo University of Science, Kagurazaka, Shinjuku-ku, Tokyo 162-8601, Japan

3 Center for Space System Innovation, Tokyo University of Science, Yamazaki, Noda, Chiba 278-8510, Japan

* Correspondence: negishi@rs.tus.ac.jp

+ These authors are equally contributed to this work.

Citation: Kawawaki, T.; Kawachi, M.; Yazaki, D.; Akinaga, Y.; Hirayama, D.; Negishi, Y. Development and Functionalization of Visible-LightDriven Water-Splitting Photocatalysts. Nanomaterials 2022, 12, 344. https://doi.org/10.3390/ nano12030344

Academic Editor: Chung-Li Dong

Received: 21 December 2021

Accepted: 17 January 2022

Published: 21 January 2022

Publisher's Note: MDPI stays neutral with regard to jurisdictional claims in published maps and institutional affiliations.

Copyright: (C) 2022 by the authors. Licensee MDPI, Basel, Switzerland. This article is an open access article distributed under the terms and conditions of the Creative Commons Attribution (CC BY) license (https:// creativecommons.org/licenses/by/ $4.0 /)$.

\begin{abstract}
With global warming and the depletion of fossil resources, our fossil fuel-dependent society is expected to shift to one that instead uses hydrogen $\left(\mathrm{H}_{2}\right)$ as a clean and renewable energy. To realize this, the photocatalytic water-splitting reaction, which produces $\mathrm{H}_{2}$ from water and solar energy through photocatalysis, has attracted much attention. However, for practical use, the functionality of water-splitting photocatalysts must be further improved to efficiently absorb visible (Vis) light, which accounts for the majority of sunlight. Considering the mechanism of water-splitting photocatalysis, researchers in the various fields must be employed in this type of study to achieve this. However, for researchers in fields other than catalytic chemistry, ceramic (semiconductor) materials chemistry, and electrochemistry to participate in this field, new reviews that summarize previous reports on water-splitting photocatalysis seem to be needed. Therefore, in this review, we summarize recent studies on the development and functionalization of Vis-light-driven water-splitting photocatalysts. Through this summary, we aim to share current technology and future challenges with readers in the various fields and help expedite the practical application of Vis-light-driven water-splitting photocatalysts.
\end{abstract}

Keywords: visible-light-driven photocatalyst; cocatalyst; water splitting; carbon neutral; metal nanocluster; nanoparticle; functionalization

\section{Introduction \\ 1.1. Water-Splitting Photocatalysts}

With the increasing threat of global warming and the depletion of fossil resources, society is expected to shift to using clean and renewable energy instead of fossil fuels. Hydrogen $\left(\mathrm{H}_{2}\right)$ does not emit carbon dioxide or other harmful materials when used in energy generation. In addition to producing energy in combustion engines, $\mathrm{H}_{2}$ can also be directly converted into electric power by fuel cells (Figure $1 \mathrm{~A}$ ). If $\mathrm{H}_{2}$ can be produced via a photocatalytic water-splitting reaction [1-43], renewable energy can be produced from sunlight and water, which are abundant on Earth (Figure 1B). Therefore, watersplitting photocatalysts have attracted huge attention as a means to address energy and environmental problems.

However, to realize the practical use of water-splitting photocatalysts, solar-to-hydrogen conversion efficiency (STH) should be improved to about 10\%. As shown in Figure 2, it is difficult to achieve STH $=10 \%$ using only ultraviolet (UV) light [44]. Therefore, the use of visible (Vis)-light-driven water-splitting photocatalysts that conduct water splitting under 
Vis light (400 $\mathrm{nm} \leq \lambda \leq 800 \mathrm{~nm}$ ), which accounts for most sunlight, is essential to achieve this goal [45-48].

(A)

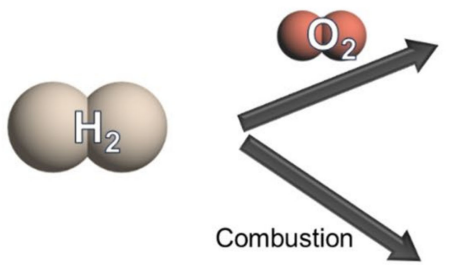

(B)

(a)

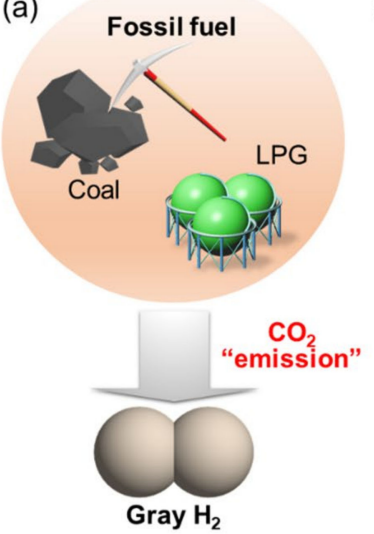

(b)

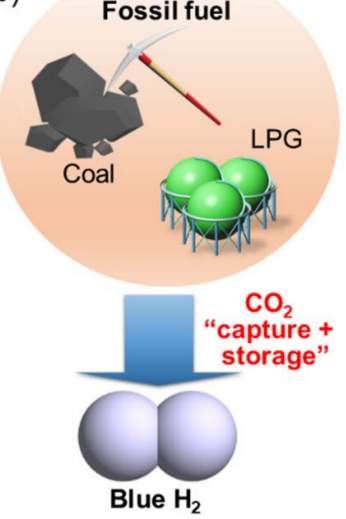

(c) Renewable + Water energy electrolysis

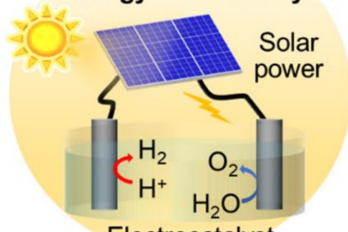

Electrocatalyst

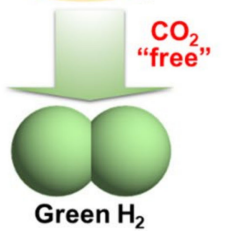

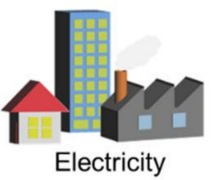

(d) Solar + Photocatalytic energy ${ }^{+}$water splitting

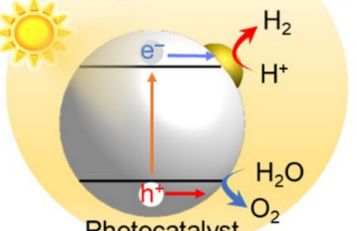

Photocatalyst

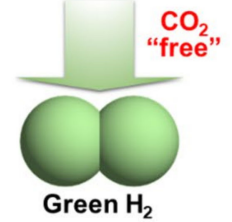

Figure 1. (A) Schematic of the expected use of $\mathrm{H}_{2}$ in a " $\mathrm{H}_{2}$-energy society". (B) Coloration of $\mathrm{H}_{2}$ production methods: (a) gray $\mathrm{H}_{2}$, (b) blue $\mathrm{H}_{2}$, and (c) green $\mathrm{H}_{2}$ produced by renewable energy, and (d) green $\mathrm{H}_{2}$ produced by water-splitting photocatalyst. LPG represents liquefied petroleum gas.

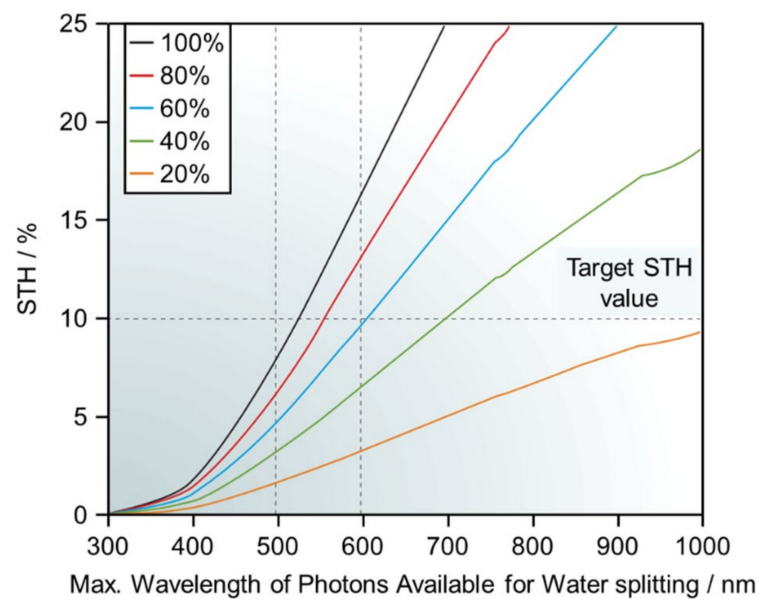

Figure 2. Different apparent quantum yields (AQYs) covering the typical range of $20-100 \%$. Dashed lines represent STH efficiencies at different AQYs with the maximum operable wavelength of 500 or $600 \mathrm{~nm}$ and the target STH value of 10\%. Associated calculations assumed AM1.5G solar irradiance. Reproduced with permission from reference [44]. Copyright 2017 Springer Nature Limited.

\subsection{Development of Vis-Light-Driven Water-Splitting Photocatalysts}

When a water-splitting reaction is conducted using a semiconductor photocatalyst, the reaction consists of three main steps (Figure 3A), as follows: (1) The semiconductor photocatalyst absorbs light energy, resulting in electronic excitation from the valence band (VB) to the conduction band (CB); (2) the excited electrons and remaining holes in the VB migrate to the photocatalyst surface or cocatalyst nanoparticles (NPs), respectively; 
(3) the $\mathrm{H}_{2}$ evolution reaction (HER; Equation (1)) and oxygen $\left(\mathrm{O}_{2}\right)$ evolution reaction (OER; Equation (2)) proceed on the cocatalyst or photocatalyst surface.

$$
\begin{gathered}
2 \mathrm{H}^{+}+2 \mathrm{e}^{-} \rightarrow \mathrm{H}_{2} \\
2 \mathrm{H}_{2} \mathrm{O}+4 \mathrm{~h}^{+} \rightarrow 4 \mathrm{H}^{+}+\mathrm{O}_{2}
\end{gathered}
$$
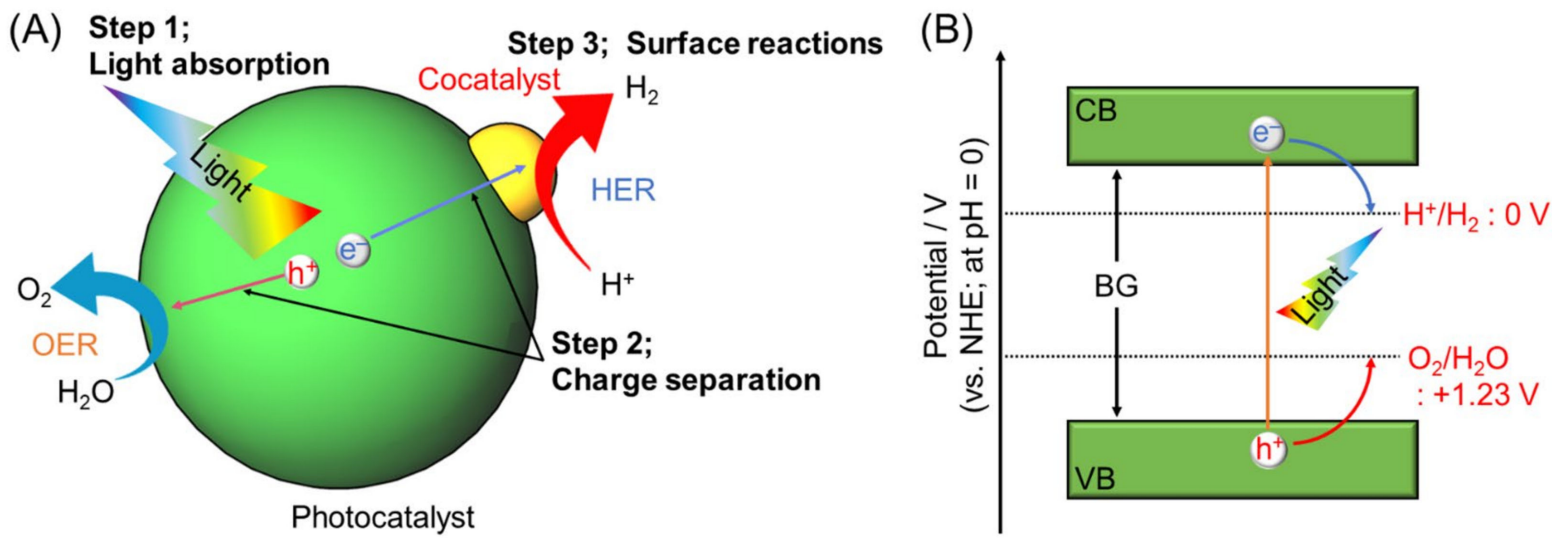

Figure 3. (A) Schematic of photocatalytic water-splitting reactions: Step 1, light absorption; Step 2, charge separation; and Step 3, surface reactions. (B) Principle of water splitting using semiconductor photocatalysts. HER, OER, VB CB, and BG represent hydrogen evolution reaction, oxygen evolution reaction, valence band, conduction band, and band gap, respectively.

Theoretically, $\mathrm{H}_{2} \mathrm{O}$ reduction and $\mathrm{H}_{2}$ evolution proceed when the $\mathrm{CB}$ minimum edge (CBM) of the semiconductor photocatalyst is more negative than the reduction potential of $\mathrm{H}_{2} \mathrm{O}\left(0 \mathrm{~V}\right.$ vs. normal $\mathrm{H}_{2}$ electrode (NHE); $\mathrm{pH}=0$ ). The $\mathrm{H}_{2} \mathrm{O}$ oxidation reaction proceeds, and $\mathrm{O}_{2}$ is evolved when the $\mathrm{VB}$ maximum edge $(\mathrm{VBM})$ of the semiconductor photocatalyst is more positive than the oxidation potential of $\mathrm{H}_{2} \mathrm{O}(1.23 \mathrm{~V}$ vs. NHE; $\mathrm{pH}=0)$ (Figure 3B). When the band gap (BG) of the semiconductor photocatalyst is sufficiently wide (UV-lightdriven photocatalyst), such control of the CBM and VBM positions is relatively easy. In contrast, when the BG of the semiconductor photocatalyst is narrow (Vis-light-driven watersplitting photocatalyst), achieving appropriate CBM and VBM positions simultaneously becomes difficult. Furthermore, a semiconductor photocatalyst with a CBM and VBM that satisfy the above conditions is not guaranteed to achieve an overall water-splitting reaction (OWSR). This is due to the following factors: (i) The high activation energy of the water-splitting reaction makes it difficult for the reaction to proceed; (ii) recombination of electrons and holes (excitons) causes the reaction to be deactivated; and (iii) generated $\mathrm{H}_{2}$ and $\mathrm{O}_{2}$ cause a reverse reaction. Therefore, only a few studies have reported one-step photocatalytic materials that can achieve an OWSR under Vis light (Figure 4A) [49]. 
(A) One-step photoexcitation system (B) Two-step (Z-scheme) photoexcitation system
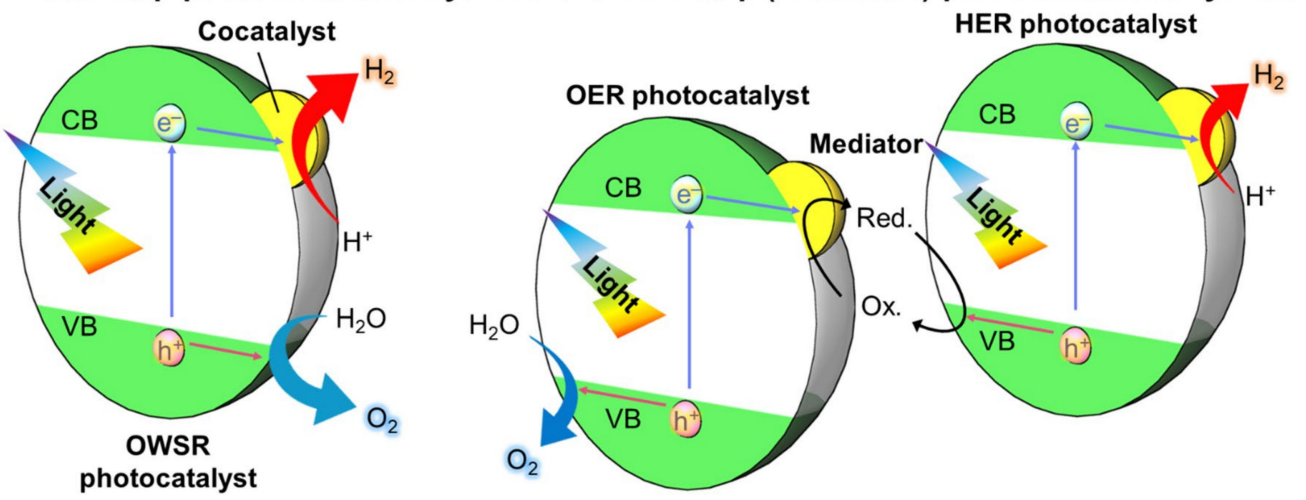

Figure 4. Schematic illustrations of photocatalytic reactions: (A) one-step photoexcitation system for overall water-splitting reaction (OWSR); (B) two-step (Z-scheme) photoexcitation system for OWSR. Red. and Ox. represent reductants and oxidants, respectively.

The OWSR can also be achieved by combining two semiconductor photocatalysts, which can conduct the half-reactions of water splitting (HER and OER), and a redox couple (mediator), which can transfer excitons between them (Figure 4B) [50,51]. This two-step reaction system, which imitates plant photosynthesis, is called the Z-scheme water-splitting reaction. In this reaction, any semiconductor that can cause a half-reaction can be used. Therefore, the number of available photocatalysts in this reaction is much larger than in the one-step water-splitting reaction, and longer-wavelength light can be used. As the HER and OER occur on two separate photocatalysts, the reverse reaction of $\mathrm{H}_{2}$ and $\mathrm{O}_{2}$ evolution can be suppressed in the Z-scheme system using a two-port H-type electrolytic cell with an ion-exchange membrane. Furthermore, separation of the evolved gases is not necessary using this method. However, the reverse reaction involving the redox couples can occur in this system, which does not occur in the one-step water-splitting reaction [51]. Furthermore, as a disadvantage, the theoretical STH of the Z-scheme water-splitting reaction is lower than that of the one-step water-splitting reaction, as two photons are required in a single reaction.

Unfortunately, at present, there is no Vis-light-driven water-splitting photocatalyst that can realize practical application in either the one-step or Z-scheme reaction. To generate sufficient $\mathrm{H}_{2}$ to withstand market competition using water-splitting photocatalysts, and thereby realize a $\mathrm{H}_{2}$-energy society, it is essential to greatly improve the functionality of Vis-light-driven photocatalysts in the future.

\subsection{Purpose of This Review}

Considering the mechanism of water-splitting photocatalysis (Figure 3), researchers in the fields of catalytic chemistry, ceramic (semiconductor) materials chemistry, electrochemistry, metal NP/nanocluster (NC) chemistry [52-68], surface spectroscopy [69-71], and theoretical chemistry [72] must be employed to create highly functional Vis-lightdriven water-splitting photocatalysts. Actually, we specialize in the chemical composition/structure control of metal NCs and have succeeded in enhancing the functionality of some UV-light-driven water-splitting photocatalysts by applying these techniques to water-splitting photocatalysts [73-79]. However, for researchers in metal NP/NC chemistry, surface spectroscopy, and theoretical chemistry, among other fields, to participate in this field, new reviews that summarize previous reports on water-splitting photocatalysis seem to be needed. Therefore, this review summarizes recent reports on the development and functionalization of Vis-light-driven water-splitting photocatalysts, and future issues are discussed. Through this summary, we aim to share the current technology and future issues with readers not previously involved in water-splitting photocatalysis and help expedite the practical application of Vis-light-driven water-splitting photocatalysts. 


\subsection{Structure of This Review}

The outline of this review is as follows. Section 2 describes the methods used to develop Vis-light-driven water-splitting photocatalysts. Specifically, Section 2.1 describes the fabrication of Vis-light-driven water-splitting photocatalysts by modifying the BG of metal oxide water-splitting photocatalysts (UV-light-driven water-splitting photocatalyst) (Figure 5A), and Section 2.2 describes the development of Vis-light-driven water-splitting photocatalysts using materials other than metal oxides (Figure 5B). Section 3 describes controlling the cocatalyst (Figure 3A), which operates as the active site (Figure 5C). Section 4 describes means for separating each reaction site (Figure 5D) to enhance the efficiency of the water-splitting reaction. Section 5 provides a short summary and, finally, Section 6 describes our future outlook.

\section{Development of Vis-light-driven water-splitting semiconductor photocatalysts}

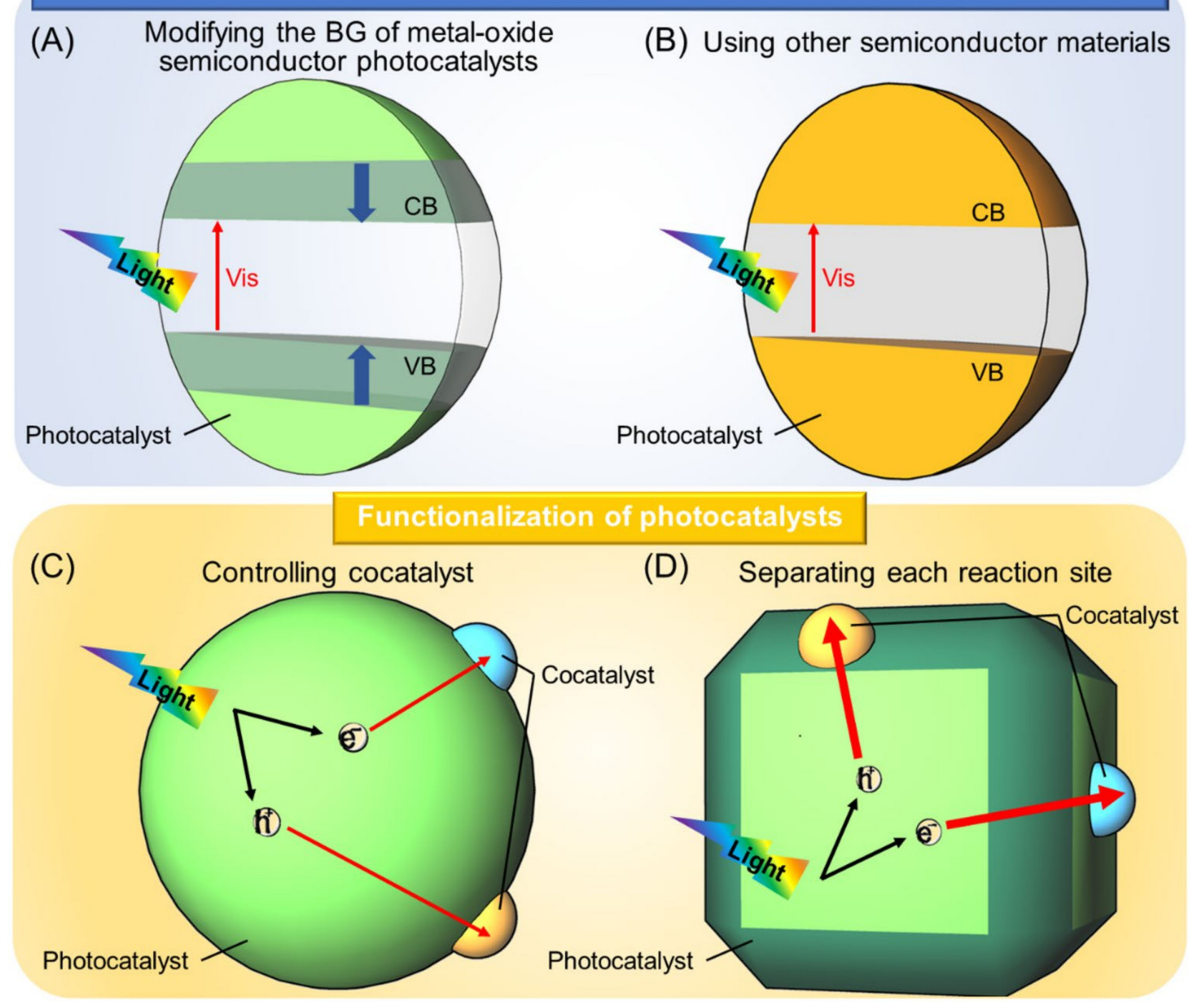

Figure 5. Schematic illustrations of the contents of Sections $2-4$ in this review. Creation of Vislight-driven water-splitting semiconductor photocatalysts by (A) modifying the BG of metal oxide semiconductor photocatalysts (UV-light-driven water-splitting photocatalysts; Section 2.1) and (B) using other semiconductor materials (Section 2.2). Functionalization of photocatalysts by (C) controlling the cocatalysts (Section 3) and (D) separating each reaction site (Section 4).

In this review, we have categorized methods for the fabrication and functionalization of Vis-light-driven water-splitting photocatalysts according to our viewpoint. Therefore, this categorization might differ from previous reviews $[44,49,51,80-90]$. Furthermore, as this review was written for readers not previously involved in water-splitting photocatalysis, methods used to prepare each photocatalyst and analyze their properties are not described. Therefore, readers interested in details of these experiments are referred to the original papers cited. 


\section{Creation of Vis-Light-Driven Water-Splitting Semiconductor Photocatalysts}

2.1. Modification of BG of Metal Oxide Semiconductor Photocatalysts (UV-Light-Driven Water-Splitting Photocatalysts)

As metals form strong bonds with $\mathrm{O}$, metal oxides are highly stable. Therefore, early studies on water-splitting semiconductor photocatalysts have mainly used metal oxides as photocatalytic materials. Such research has shown that metal oxides with an electronic structure of $\mathrm{d}^{0}$ (transition metal ions, such as $\mathrm{Ti}^{4+}$, zirconium ion $\left(\mathrm{Zr}^{4+}\right)$, niobium ion $\left(\mathrm{Nb}^{5+}\right)$, tantalum ion $\left(\mathrm{Ta}^{5+}\right)$, vanadium ion $\left(\mathrm{V}^{5+}\right)$, tungsten ion $\left(\mathrm{W}^{6+}\right)$, and cerium ion $\left.\left(\mathrm{Ce}^{4+}\right)\right)$ and $\mathrm{d}^{10}$ (typical metal ions, such as zinc ion $\left(\mathrm{Zn}^{2+}\right)$, indium ion $\left(\mathrm{In}^{3+}\right)$, gallium ion $\left(\mathrm{Ga}^{3+}\right)$, germanium ion $\left(\mathrm{Ge}^{4+}\right)$, tin ion $\left(\mathrm{Sn}^{4+}\right)$, and antimony ion $\left.\left(\mathrm{Sb}^{5+}\right)\right)$, possess functions as watersplitting photocatalysts. However, as most of these metal oxides have a BG of more than $3 \mathrm{eV}$, they can cause an OWSR only when irradiated with UV light. Therefore, to cause a water-splitting reaction under Vis light, the BG of the semiconductor photocatalyst must be narrowed to match the energy of the Vis-light region $(\mathrm{BG}<3.0 \mathrm{eV})$.

The VB of metal oxide semiconductor photocatalysts consists of $\mathrm{O} 2 \mathrm{p}$ orbitals, with the VBM located at approx. $+3 \mathrm{~V}$ (vs. NHE; at $\mathrm{pH}=0$ ) in many cases [91]. Four methods have been reported for modifying electronic structures to cause these semiconductor photocatalysts to absorb Vis light, as follows: (i) shifting the energy position of the VBM to the negative-potential side by substituting anions or metal cations (Section 2.1.1; Figure 6A) [92-118]; (ii) formation of impurity levels in the BG by doping (Section 2.1.2; Figure 6B) [119-128]; (iii) narrowing the BG itself through solid-solution formation (Section 2.1.3; Figure 6C) [129-133]; and (iv) shifting the whole band structure to the negative-potential side by reducing particle size (Section 2.1.4; Figure 6D) [134,135]. In this section, typical studies using each of these four methods are described. The BG energy, type and loading amount of appropriate cocatalysts, possible reactions (OWSR, HER, or OER; Figure 4), activity, and references for semiconductor photocatalysts described in this section are summarized in Figure 7 and Table 1 [51,87,90-103,105-107,109,110,112,114,116-120,128-132,134].

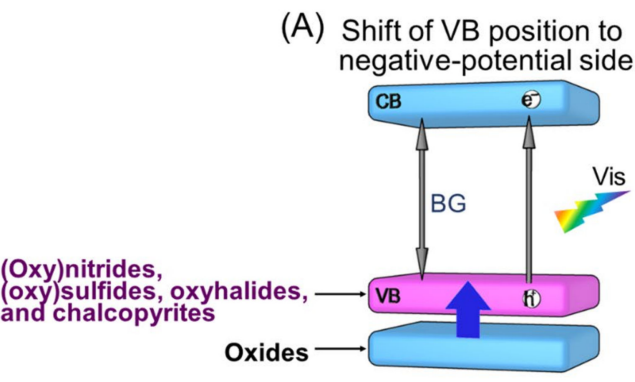

(C) Narrowing of BG by solid-solution formation

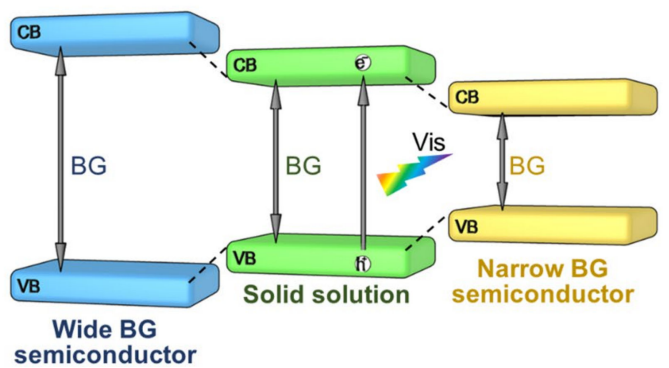

(B) Formation of

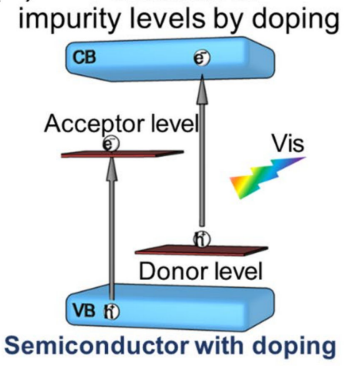

(D)

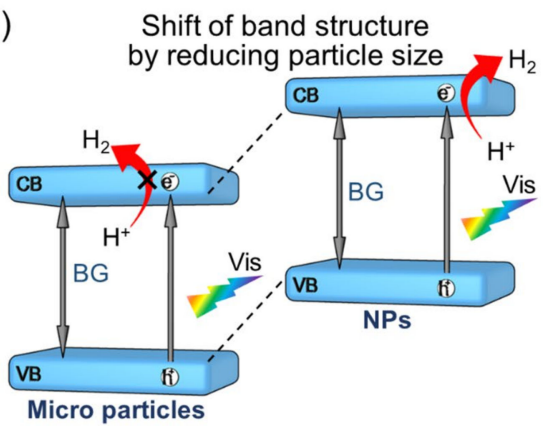

Figure 6. Schematic illustrations showing strategies for obtaining Vis-light-driven water-splitting photocatalysts. (A) Shift of VB position to negative-potential side, (B) formation of impurity levels by doping, (C) narrowing of BG by solid-solution formation, and (D) shift of band structure by reducing particle size. 


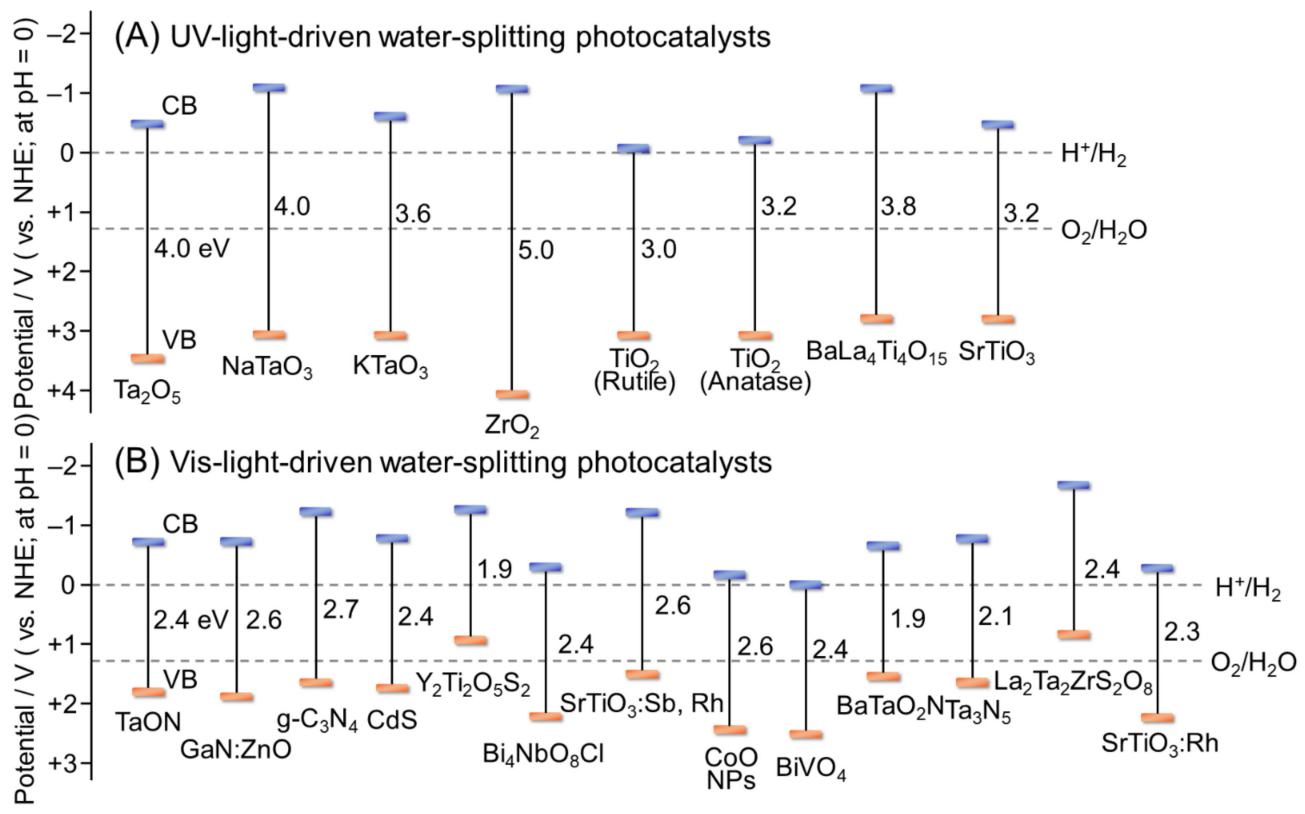

Figure 7. Band structures of (A) UV-light-driven and (B) Vis-light-driven water-splitting photocatalysts. In this figure, only semiconductors for which the band structure at $\mathrm{pH}=0$ has been reported are described. For the BG values of these and other semiconductors, please also see Table 1 [51,87,90-99].

Table 1. BG, Effective cocatalysts, possible reaction, and efficiency of Vis-light-driven water-splitting photocatalysts introduced in Section 2.1.

\begin{tabular}{|c|c|c|c|c|c|}
\hline Photocatalyst & $\begin{array}{l}\mathrm{BG} \\
/ \mathrm{eV}\end{array}$ & $\begin{array}{c}\text { Cocatalyst } \\
(/ \mathbf{w t} \%)\end{array}$ & Possible Reaction ${ }^{a}$ & Efficiency $^{b}$ & Ref. \\
\hline $\mathrm{Ag}(\mathrm{I})-\mathrm{Ga}_{2} \mathrm{In}_{3} \mathrm{~S}_{8}$ & 2.3 & $\mathrm{Rh}(0.5)$ & HER & $\mathrm{AQY}=15 \%$ (at $460 \mathrm{~nm})$ & [116] \\
\hline $\mathrm{Ag}(\mathrm{I})-\mathrm{K}_{2} \mathrm{SrTa}_{2} \mathrm{O}_{7}$ & 2.8 & Bare & OER & $\begin{array}{c}\mathrm{ER}=3.0 \mu \mathrm{mol} \mathrm{h}^{-1} \\
(>420 \mathrm{~nm})\end{array}$ & [117] \\
\hline $\mathrm{Ag}(\mathrm{I})-\mathrm{Li}_{2} \mathrm{SrTa}_{2} \mathrm{O}_{7}$ & 2.8 & Bare & OER & $\begin{array}{c}\mathrm{ER}=4.0 \mu \mathrm{mol} \mathrm{h}^{-1} \\
(>420 \mathrm{~nm})\end{array}$ & [117] \\
\hline $\mathrm{Bi}_{4} \mathrm{NbO}_{8} \mathrm{Cl}$ & 2.4 & $\mathrm{Pt}(0.5)$ & $\begin{array}{c}\text { OER } \\
(\mathrm{pH}=2)\end{array}$ & $\mathrm{AQY}=0.4 \%($ at $420 \mathrm{~nm})$ & [110] \\
\hline $\mathrm{Bi}_{4} \mathrm{TaO}_{8} \mathrm{Br}$ & 2.5 & $\mathrm{RuO}_{2}(0.7)$ & OER & $\mathrm{AQY}>20 \%$ (at $420 \mathrm{~nm}$ ) & [111] \\
\hline $\mathrm{Bi}_{4} \mathrm{NbO}_{8} \mathrm{Br}$ & 2.5 & $\mathrm{~N} / \mathrm{A}$ & $\mathrm{N} / \mathrm{A}$ & $\mathrm{N} / \mathrm{A}$ & [109] \\
\hline $\mathrm{BiOCl}$ & 3.4 & $\mathrm{~N} / \mathrm{A}$ & $\mathrm{N} / \mathrm{A}$ & $\mathrm{N} / \mathrm{A}$ & [109] \\
\hline $\mathrm{BiOBr}$ & 2.8 & $\mathrm{~N} / \mathrm{A}$ & $\mathrm{N} / \mathrm{A}$ & $\mathrm{N} / \mathrm{A}$ & [109] \\
\hline $\mathrm{BiOI}$ & 1.8 & $\mathrm{~N} / \mathrm{A}$ & $\mathrm{N} / \mathrm{A}$ & $\mathrm{N} / \mathrm{A}$ & [109] \\
\hline $\mathrm{BiVO}_{4}$ & 2.4 & Bare & OER & $\mathrm{AQY}=9.0 \%$ (at $450 \mathrm{~nm})$ & [112] \\
\hline$m-\mathrm{BiVO}_{4}: \mathrm{In}, \mathrm{Mo}$ & 2.5 & $\mathrm{RuO}_{2}(3.0)$ & $\begin{array}{c}\text { OWSR } \\
(\mathrm{pH}=7)\end{array}$ & $\begin{array}{c}\mathrm{AQY}=3.2 \% \\
(\text { at } 420-800 \mathrm{~nm})\end{array}$ & [128] \\
\hline CdS-P & 1.8 & $\mathrm{CoP}(5.0)$ & HER & $\mathrm{N} / \mathrm{A}$ & [107] \\
\hline CoO NPs & 2.6 & Bare & OWSR & $\mathrm{STH}=\sim 5.0 \%(\mathrm{AM} 1.5 \mathrm{G})$ & [134] \\
\hline $\mathrm{Cu}(\mathrm{I})-\mathrm{Ga}_{2} \mathrm{In}_{3} \mathrm{~S}_{8}$ & 1.9 & $\mathrm{Rh}(1.5)$ & HER & $\mathrm{AQY}=15 \%($ at $560 \mathrm{~nm})$ & [116] \\
\hline $\mathrm{Cu}(\mathrm{I})-\mathrm{K}_{2} \mathrm{La}_{2} \mathrm{Ta}_{3} \mathrm{O}_{10}$ & 2.0 & $\mathrm{Ru}(0.3)$ & HER & $\begin{array}{c}\mathrm{ER}=45 \mu \mathrm{mol} \mathrm{h}^{-1} \\
(>420 \mathrm{~nm})\end{array}$ & [117] \\
\hline $\mathrm{Cu}(\mathrm{I})-\mathrm{K}_{2} \mathrm{SrTa}_{2} \mathrm{O}_{7}$ & 2.1 & $\mathrm{Ru}(0.3)$ & HER & $\begin{array}{c}\mathrm{ER}=66 \mu \mathrm{mol} \mathrm{h}^{-1} \\
\quad(>420 \mathrm{~nm})\end{array}$ & [117] \\
\hline GaN:ZnO & 2.6 & $\mathrm{RuO}_{2}(5.0)$ & OWSR (pH = 3) & $\begin{array}{c}\mathrm{AQY}=0.14 \% \\
(\text { at } 300-480 \mathrm{~nm})\end{array}$ & [129] \\
\hline
\end{tabular}


Table 1. Cont.

\begin{tabular}{|c|c|c|c|c|c|}
\hline Photocatalyst & $\begin{array}{l}\mathrm{BG} \\
/ \mathrm{eV}\end{array}$ & $\begin{array}{c}\text { Cocatalyst } \\
(/ \mathbf{w t} \%)\end{array}$ & Possible Reaction ${ }^{a}$ & Efficiency $^{b}$ & Ref. \\
\hline & & $\begin{array}{c}\mathrm{Rh}_{2-x} \mathrm{Cr}_{x} \mathrm{O}_{3} \\
\text { (Rh: } 1.0, \mathrm{Cr}: 1.5)\end{array}$ & $\begin{array}{c}\text { OWSR } \\
(\mathrm{pH}=4.5)\end{array}$ & $\begin{array}{c}\mathrm{AQY}=5.9 \% \\
(\text { at } 420-440 \mathrm{~nm})\end{array}$ & [130] \\
\hline $\mathrm{La}_{5} \mathrm{Ti}_{2} \mathrm{Cu}_{0.9} \mathrm{Ag}_{0.1} \mathrm{~S}_{5} \mathrm{O}_{7}: \mathrm{Ga}$ & 1.8 & $\mathrm{Cr}_{2} \mathrm{O}_{3} / \mathrm{Rh}$ & $\begin{array}{c}\text { OWSR } \\
(\mathrm{pH}=10) \\
\left(-\mathrm{Au}-\mathrm{BiVO}_{4}\right)\end{array}$ & $\begin{array}{c}\mathrm{AQY}=4.9 \%(>420 \mathrm{~nm}) \\
\mathrm{STH}=0.11 \%\end{array}$ & [131] \\
\hline $\mathrm{La}_{5} \mathrm{Ti}_{2} \mathrm{CuS}_{5} \mathrm{O}_{7}$ & 1.9 & $\begin{array}{c}\operatorname{Pt}(0.3) \\
-\mathrm{NiS}(1.0)\end{array}$ & $\begin{array}{c}\text { HER } \\
(\mathrm{pH}=10)\end{array}$ & $\begin{array}{c}A Q Y=1.8 \% \\
(\text { at } 420 \pm 10 \mathrm{~nm})\end{array}$ & {$[105,106]$} \\
\hline $\mathrm{La}_{5} \mathrm{Ti}_{2} \mathrm{Cu}\left(\mathrm{S}_{1-x} \mathrm{Se}_{x}\right)_{5} \mathrm{O}_{7}$ & $1.6-1.9$ & $\begin{array}{c}\mathrm{NiS} \\
(0.5 \text { or } 1.0)\end{array}$ & $\begin{array}{c}\text { HER } \\
(\mathrm{pH}=12)\end{array}$ & $\mathrm{N} / \mathrm{A}$ & [132] \\
\hline $\mathrm{La}_{2} \mathrm{Ta}_{2} \mathrm{ZrS}_{2} \mathrm{O}_{8}$ & 2.4 & $\mathrm{IrO}_{2}(0.5)$ & $\begin{array}{c}\text { OER } \\
(\mathrm{pH}=13)\end{array}$ & $\mathrm{N} / \mathrm{A}$ & [102] \\
\hline $\mathrm{Cu}(\mathrm{I})-\mathrm{NaTaO}_{3}$ & 2.0 & $\mathrm{Ru}(0.3)$ & HER & $\begin{array}{c}\mathrm{AQY}=0.18 \% \\
(>420 \mathrm{~nm})\end{array}$ & [114] \\
\hline $\mathrm{Ag}(\mathrm{I})-\mathrm{Na}_{2} \mathrm{~W}_{4} \mathrm{O}_{13}$ & 2.9 & $\mathrm{~N} / \mathrm{A}$ & $\begin{array}{c}\text { OER } \\
(\mathrm{pH}=2.4)\end{array}$ & $\begin{array}{c}\mathrm{ER}=0.9 \mu \mathrm{mol} \mathrm{h}-1 \\
(>420 \mathrm{~nm})\end{array}$ & [118] \\
\hline $\mathrm{Sm}_{2} \mathrm{Ti}_{2} \mathrm{~S}_{2} \mathrm{O}_{5}$ & 2.1 & $\mathrm{Pt}(1.0)$ & $\begin{array}{c}\text { HER } \\
(\mathrm{pH}=8-9)\end{array}$ & $\begin{array}{c}\mathrm{ER}=22 \mu \mathrm{mol} \mathrm{h}^{-1} \\
(440 \mathrm{~nm} \leq \lambda \leq 650 \mathrm{~nm})\end{array}$ & {$[100,109]$} \\
\hline & & & $\begin{array}{c}\text { OER } \\
(\mathrm{pH}=10)\end{array}$ & $\begin{array}{c}\mathrm{ER}=22 \mu \mathrm{mol} \mathrm{h}^{-1} \\
(440 \mathrm{~nm} \leq \lambda \leq 650 \mathrm{~nm})\end{array}$ & [101] \\
\hline $\mathrm{SrTiO}_{3}: \mathrm{Rh}$ & 1.7 & $\mathrm{Pt}(0.1)$ & HER & $\mathrm{AQY}=5.2 \%($ at $420 \mathrm{~nm})$ & [119] \\
\hline $\mathrm{SrTiO}_{3}: \mathrm{Sb}, \mathrm{Rh}$ & $\mathrm{N} / \mathrm{A}$ & $\mathrm{InO}_{2}(3.0)$ & $\begin{array}{c}\text { HER } \\
(\mathrm{pH}=3)\end{array}$ & $\mathrm{AQY}=0.1 \%($ at $420 \mathrm{~nm})$ & [120] \\
\hline \multirow[t]{4}{*}{$\mathrm{TaON}$} & 2.4 & $\mathrm{Pt}(3.0)$ & HER & $\mathrm{AQY}=0.2 \%($ at $420 \mathrm{~nm})$ & {$[92,94]$} \\
\hline & & $\mathrm{Pt}(3.0)$ & $\begin{array}{c}\text { OER } \\
(\mathrm{pH}=\sim 8)\end{array}$ & $\mathrm{AQY}=34 \%($ at $420 \mathrm{~nm})$ & [94] \\
\hline & & $\mathrm{Ru}(0.05)$ & HER & $\begin{array}{c}\mathrm{ER}=120 \mu \mathrm{mol} \mathrm{h}^{-1} \\
(>420 \mathrm{~nm})\end{array}$ & {$[103,107]$} \\
\hline & & $\begin{array}{l}\mathrm{Cr}_{2} \mathrm{O}_{3}(2.5) \\
/ \mathrm{RuO}_{x}(3.0) \\
-\mathrm{IrO}_{2}(4.0)\end{array}$ & OWSR & $\mathrm{AQY}<0.1 \%$ (at $420 \mathrm{~nm})$ & \\
\hline \multirow[t]{2}{*}{$\mathrm{Ta}_{3} \mathrm{~N}_{5}$} & 2.1 & $\mathrm{Pt}(3.0)$ & HER & $\begin{array}{c}\mathrm{AQY}=0.1 \% \\
(\text { at } 420-600 \mathrm{~nm})\end{array}$ & {$[92,97]$} \\
\hline & & $\begin{array}{l}\mathrm{Cr}_{2} \mathrm{O}_{3}(2.5) \\
/ \mathrm{RuO}_{x}(3.0) \\
-\mathrm{IrO}_{2}(4.0)\end{array}$ & $\begin{array}{c}\text { OER } \\
(\mathrm{pH}=8.5)\end{array}$ & $\begin{array}{c}\mathrm{AQY}=10 \% \\
(\text { at } 420-600 \mathrm{~nm})\end{array}$ & [98] \\
\hline $\mathrm{Y}_{2} \mathrm{Ti}_{2} \mathrm{O}_{5} \mathrm{~S}_{2}$ & 1.9 & $\begin{array}{l}\mathrm{Cr}_{2} \mathrm{O}_{3}(1.5) \\
/ \mathrm{Rh}(2.0) \\
-\mathrm{IrO}_{2}(0.3)\end{array}$ & $\begin{array}{c}\text { OWSR } \\
(\mathrm{pH}=8.5)\end{array}$ & $\begin{array}{l}\operatorname{AQY}\left(\mathrm{H}_{2}\right)=5.3 \pm 0.3 \% \\
\quad(\text { at } 420-480 \mathrm{~nm}) \\
\mathrm{AQY}\left(\mathrm{O}_{2}\right)=2.3 \pm 0.1 \% \\
\quad(\text { at } 420-480 \mathrm{~nm})\end{array}$ & [103] \\
\hline
\end{tabular}

${ }^{a}$ OER, HER, and OWSR represent the oxygen evolution reaction, hydrogen evolution reaction, and overall watersplitting reaction, respectively. ${ }^{b} \mathrm{AQY}, \mathrm{ER}, \mathrm{STH}$, and AM1.5G represent the apparent quantum yield, evolution rate, solar-to-hydrogen conversion efficiency, and global standard solar spectrum (AM1.5G), respectively.

\subsubsection{Shift of VB Position to Negative-Potential Side}

Substitution of $\mathrm{O}^{2-}$ Anion by Nitrogen, Sulfur, or Halide Anion

When the $\mathrm{O}^{2-}$ of a metal oxide is substituted by a nitrogen anion $\left(\mathrm{N}^{3-}\right)$, sulfur anion $\left(\mathrm{S}^{2-}\right)$, halide anion (chloride anion $\left(\mathrm{Cl}^{-}\right)$, bromide anion $\left(\mathrm{Br}^{-}\right)$, or iodide anion $\left(\mathrm{I}^{-}\right)$), a new $\mathrm{VB}$ can be constructed on the negative-potential side of the $\mathrm{O} 2 \mathrm{p}$ orbital of the metal oxide.

Figure $8 \mathrm{~A}$ shows the band structure of metal oxide $\mathrm{Ta}_{2} \mathrm{O}_{5}$ and $\mathrm{TaON}$, whose $\mathrm{O}^{2-}$ were substituted by $\mathrm{N}^{3-}$ (Figure 7 and Table 1) [92]. The VBM of $\mathrm{Ta}_{2} \mathrm{O}_{5}$ consists of $\mathrm{O} 2 \mathrm{p}$ orbitals and is located at $+3.4 \mathrm{~V}$ (vs. NHE; at $\mathrm{pH}=0$ ). In contrast, in TaON, $\mathrm{N} 2 \mathrm{p}$ orbitals also contribute to VB formation, shifting the VBM to $+2.1 \mathrm{~V}$ (vs. NHE; at $\mathrm{pH}=0$ ). The VBM shifts further to $+1.7 \mathrm{~V}$ (vs. $\mathrm{NHE}$; at $\mathrm{pH}=0$ ) when $\mathrm{Ta}_{3} \mathrm{~N}_{5}$ without $\mathrm{O}$ is allowed to form. $\mathrm{TaON}$ and $\mathrm{Ta}_{3} \mathrm{~N}_{5}$ can absorb light up to about 520 and $600 \mathrm{~nm}$, respectively, and are capable of the OWSR under Vis-light irradiation (Figure 7 and Table 1) [93]. 
(A)

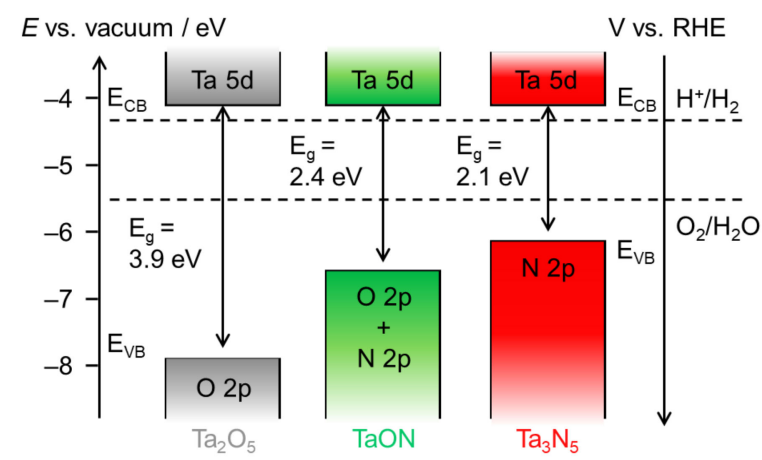

(C)
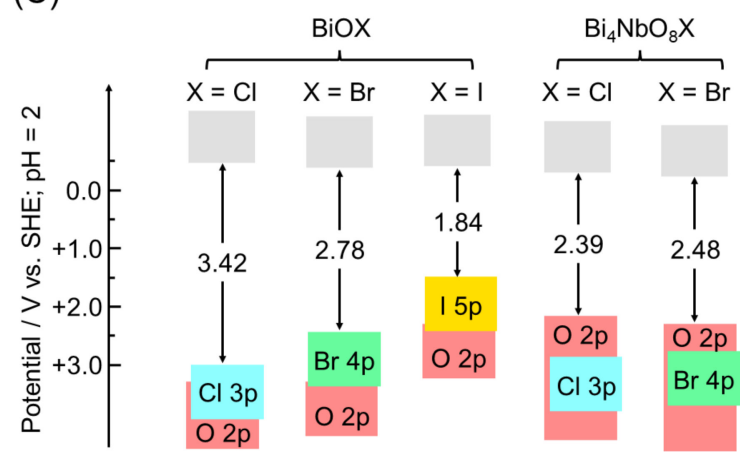

(B)

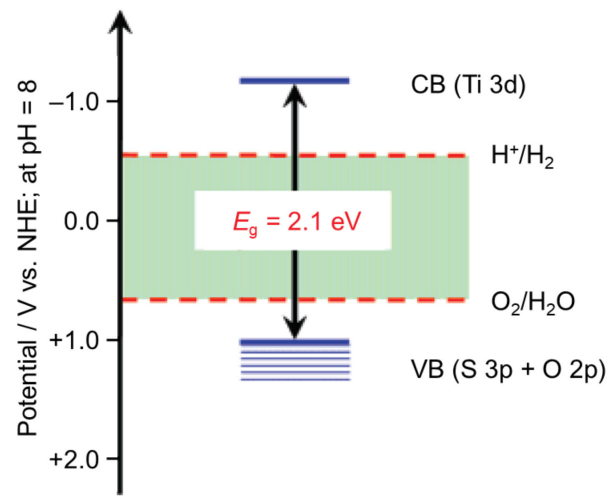

(D)

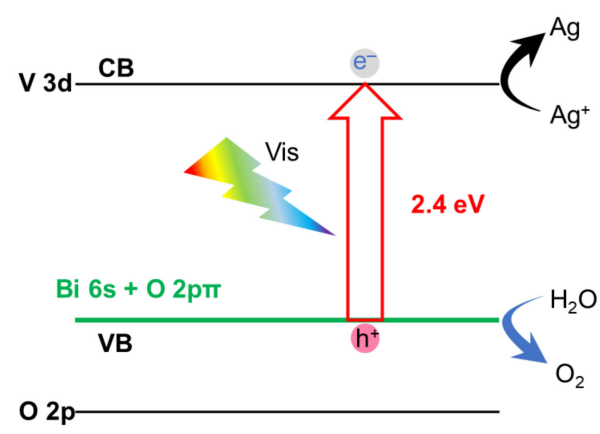

Figure 8. (A) Band diagrams for $\mathrm{Ta}_{2} \mathrm{O}_{5}, \mathrm{TaON}$, and $\mathrm{Ta}_{3} \mathrm{~N}_{5}$ showing the shift in $\mathrm{VBM}$ upon nitridation. RHE represents the reversible hydrogen electrode. (B) Schematic illustration of band edge potentials of $\mathrm{Sm}_{2} \mathrm{Ti}_{2} \mathrm{~S}_{2} \mathrm{O}_{5}$. (C) Schematic illustration of $\mathrm{VB}$ and $\mathrm{CB}$ structures of $\mathrm{BiOX}(\mathrm{X}=\mathrm{Cl}, \mathrm{Br}$, or I) and $\mathrm{Bi}_{4} \mathrm{NbO}_{8} \mathrm{X}(\mathrm{X}=\mathrm{Cl}$ or $\mathrm{Br})$. Red, blue, green, and orange boxes represent the bands from $\mathrm{O} 2 \mathrm{p}, \mathrm{Cl} 3 \mathrm{p}, \mathrm{Br}$ $4 p$, and I 5p orbitals, respectively, and the gray box shows a CB composed mainly of Bi 6p. (D) Band structures of $m-\mathrm{BiVO}_{4}$. Reproduced with permission from references $[86,92,99,109]$. Copyright 2015 American Chemical Society, 2007 American Chemical Society, 2017 American Chemical Society, and 2009 The Royal Society of Chemistry.

Meanwhile, the metal oxysulfide photocatalyst substituted with $\mathrm{S}^{2-}$ (Figure 7 and Table 1) has a VBM located on the negative-energy side compared with the metal oxide because the $\mathrm{VB}$ is formed by the hybridization of $\mathrm{S} 3 \mathrm{p}$ and $\mathrm{O} 2 \mathrm{p}$ orbitals (Figure 8B) [99-105]. For example, the $\mathrm{VBMs}$ of $\mathrm{La}_{2} \mathrm{Ta}_{2} \mathrm{ZrS}_{2} \mathrm{O}_{8}$ and $\mathrm{Y}_{2} \mathrm{Ti}_{2} \mathrm{O}_{5} \mathrm{~S}_{2}$ are located at $+0.84 \mathrm{~V}$ (vs. NHE; at $\mathrm{pH}=0$ ) and $+0.8-0.9 \mathrm{~V}$ (vs. NHE; at $\mathrm{pH}=9$ ), respectively [102,103]. Regarding metal sulfide photocatalysts, Domen and co-workers and Chen and co-workers reported that the VBM can be further raised by doping with selenium (Se) or phosphorus (P), respectively $[106,107]$.

Furthermore, in recent years, several examples of the VB being shifted to the negativepotential side by substituting $\mathrm{O}^{2-}$ with halide ions have been reported (Table 1) [108-111]. In such cases, the VBM shifts continuously to the negative-potential side with increasing electronegativity of the halide anion. For example, in bismuth oxyhalide (BiOX), the BGs of $\mathrm{BiOCl}, \mathrm{BiOBr}$, and $\mathrm{BiOI}$ are 3.42, 2.78, and $1.84 \mathrm{eV}$, respectively (Figure $8 \mathrm{C}$ ) [108,109]. In these metal halide photocatalysts, the Bi $6 \mathrm{p}$ orbital forms a CBM at a more positive position compared with the reduction potential of $\mathrm{H}_{2} \mathrm{O}$. Therefore, this photocatalyst alone cannot conduct water-splitting reactions under Vis-light irradiation. Accordingly, metal halide photocatalysts are expected to be OER photocatalysts in the Z-scheme. Regarding such metal halide photocatalysts, in 2016, Kageyama, Abe, and co-workers reported that the formation of $\mathrm{Bi}_{4} \mathrm{NbO}_{8} \mathrm{Cl}$ with $\mathrm{Nb}$ oxide enhanced its stability under Vis-light irradiation $[109,110]$.

Substitution of Metal Cation

The BG of the semiconductor can also be narrowed by introducing metal ions with a d ${ }^{10} s^{2}$-type electron configuration, such as copper ion $\left(\mathrm{Cu}^{+}\right)$, silver ion $\left(\mathrm{Ag}^{+}\right)$, lead ion 
$\left(\mathrm{Pb}^{2+}\right)$, and $\mathrm{Bi}^{3+}$, into the metal oxide photocatalyst, resulting in a widened $\mathrm{VB}$ through hybridization of their orbitals with O 2p orbitals [112-118]. For example, in 1999, Kudo and co-workers developed monoclinic $\mathrm{BiVO}_{4}\left(m-\mathrm{BiVO}_{4}\right)$ by adding $\mathrm{Bi}^{3+}$ to $\mathrm{V}$ oxide and found that the BG of this photocatalyst was narrowed to $2.4 \mathrm{eV}$ owing to $\mathrm{Bi} 6 \mathrm{~s}$ and $\mathrm{O} 2 \mathrm{p} \pi$ hybridization (Figure 8D) [112,113]. These authors also confirmed that this photocatalyst promoted $\mathrm{O}_{2}$ evolution under Vis-light irradiation in the presence of a sacrificial agent (Table 1). They also succeeded in increasing the VBM by substituting alkali metal ions with $\mathrm{Ag}^{+}$or $\mathrm{Cu}^{+}$at the near surface of bulky materials, such as $\mathrm{NaTaO}_{3}$ (Table 1) [114,115]. Furthermore, there have been several reports of Vis-light-driven water-splitting photocatalysts created by replacing alkali metal ions in the interlayer of layered oxide photocatalysts with $\mathrm{Ag}^{+}$or $\mathrm{Cu}^{+}$(Table 1) [116-118].

\subsubsection{Formation of Impurity Levels by Doping}

Metal oxide photocatalysts become Vis-light responsive by forming donor levels (impurity levels) when $0.1 \%$ to several percent of the metal ions are substituted with other transition metal ions (such as chromium ion $\left(\mathrm{Cr}^{3+}\right)$, rhodium ion $\left(\mathrm{Rh}^{3+}\right)$, and iridium ion $\left.\left(\mathrm{Ir}^{3+}\right)\right)$ while maintaining the lattice structure, known as doping. Using such a method, Kudo and co-workers succeeded in developing a Rh-doped strontium titanium oxide $\left(\mathrm{SrTiO}_{3}: \mathrm{Rh}\right)$ photocatalyst in 2004 (Figure 7) [119]. For $\mathrm{SrTiO}_{3}: \mathrm{Rh}$, a donor level (impurity level) was formed at the negative side of VBM (namely, in the forbidden band) due to doping of some $\mathrm{Ti}^{4+}$ sites with $\mathrm{Rh}^{3+}$ (Figure 9A). For $\mathrm{SrTiO}_{3}: \mathrm{Rh}$, optical absorption occurred at both 580 and $420 \mathrm{~nm}$ immediately after preparation (Figure 9B) [119]. These absorptions were attributed to impurity levels based on $\mathrm{Rh}^{4+}$ and $\mathrm{Rh}^{3+}$, respectively. However, the acceptor level (impurity level) formed by $\mathrm{Rh}^{4+}$ became an exciton recombination center [120]. Therefore, to proceed with the water-splitting reaction efficiently, $\mathrm{Rh}^{4+}$ needs to be photoreduced to $\mathrm{Rh}^{3+}$ by light irradiation [121]. Furthermore, the formation of such acceptor levels can be avoided by co-doping other metal cations to maintain the charge balance [122-124]. For example, Onishi and co-workers succeeded in suppressing the formation of $\mathrm{Rh}^{4+}$ by co-doping some $\mathrm{Ti}^{4+}$ sites with two types of ion, $\mathrm{Sb}^{5+}$ and $\mathrm{Rh}^{3+}$ (Figure 9B) [125]. In contrast, Domen and co-workers succeeded in reducing the ratio of $\mathrm{Rh}^{4+}$ and increasing the ratio of $\mathrm{Rh}^{3+}$ by doping some $\mathrm{Ti}^{4+}$ sites with $\mathrm{Rh}^{3+}$ and some $\mathrm{Sr}^{2+}$ sites with lanthanum ion $\left(\mathrm{La}^{3+}\right)$ (co-doping) [126].

Doping is also effective at shifting the positions of the CB and VB [127]. In 2015, Lee and co-workers simultaneously substituted $\mathrm{Bi}^{3+}$ and $\mathrm{V}^{5+}$ in $m$-BiVO $\mathrm{Bith}^{3+}$ and molybdenum ion $\left(\mathrm{Mo}^{6+}\right)$, respectively, to create $\mathrm{BiVO}_{4}: \mathrm{In}, \mathrm{Mo}$. In this $\mathrm{BiVO}_{4}: \mathrm{In}$, Mo photocatalyst, $\mathrm{BiVO}_{4}$ was a mixture of $m-\mathrm{BiVO}_{4}$ and tetragonal $\mathrm{BiVO}_{4}\left(t-\mathrm{BiVO}_{4}\right)$, which induced an increase in the compressive lattice strain (Figure $9 \mathrm{C}$ ). This caused an increase in the CB and allowed $\mathrm{BiVO}_{4}$ :In,Mo to promote the HER (Figure 9D) [128]. 
(A)

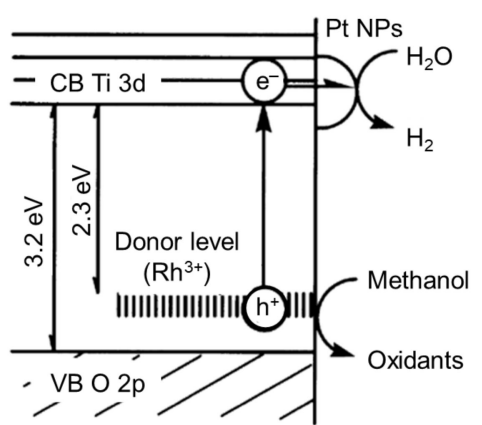

(C)

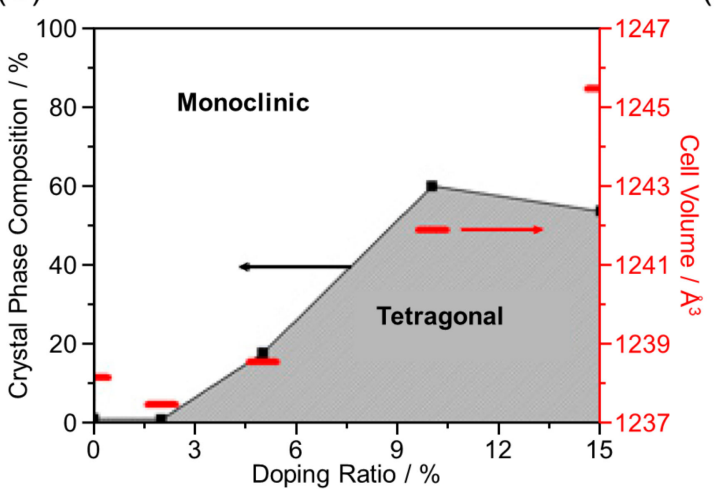

(B)

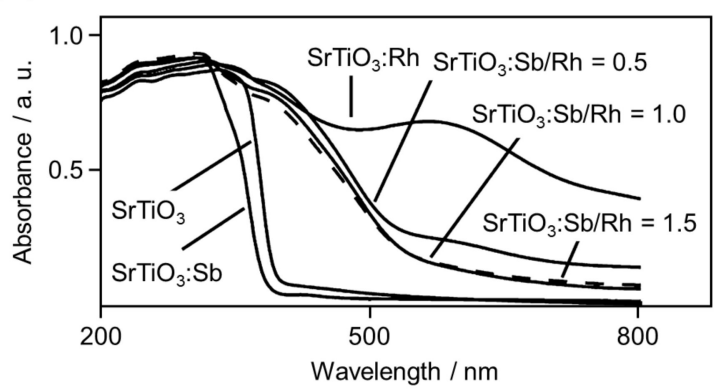

(D)

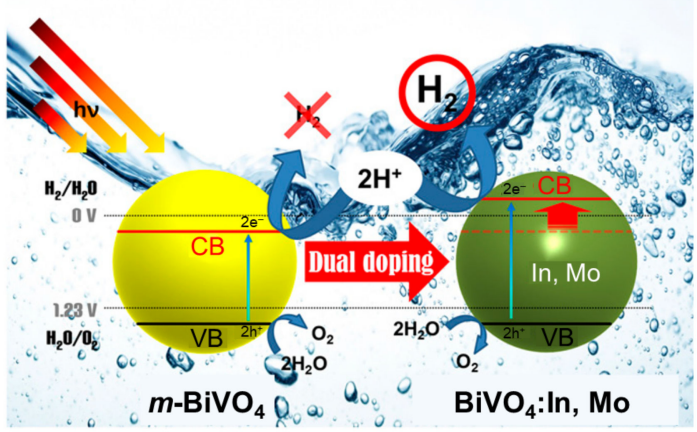

Figure 9. (A) Band structure of $\mathrm{SrTiO}_{3}: \mathrm{Rh}$ after photoreduction. (B) Diffuse reflection spectra of the six photocatalysts. For example, " $\mathrm{SrTiO}_{3}: \mathrm{Sb} / \mathrm{Rh}=0.5$ " indicates " $\mathrm{SrTiO}_{3}: \mathrm{Sb}, \mathrm{Rh}$ in which $\mathrm{Sb} / \mathrm{Rh}=0.5$ ". (C) Crystal phase diagram with calculated unit cell volume for different doping ratios (atom ratios of In and Mo) of $m-\mathrm{BiVO}_{4}$. (D) Effect of dual doping by In and Mo on the activity of $\mathrm{BiVO}_{4}$. Reproduced with permission from references [119,125,128]. Copyright 2004 American Chemical Society, 2013 American Chemical Society, and 2015 National Academy of Sciences.

\subsubsection{Narrowing of BG by Solid-Solution Formation}

Semiconductor materials with the same crystal structure can easily form a solid solution [136]. The BG and energy levels of the solid solution continuously change depending on the ratio of the two types of semiconductor material [129-133]. Domen and co-workers succeeded in creating $\mathrm{GaN}: \mathrm{ZnO}$, which provided the first example of efficient and stable Vis-light-driven OWSR (Figure 10A) by solid-solution formation from GaN (3.4 eV) and $\mathrm{ZnO}(3.2 \mathrm{eV})$, which are UV-light-driven photocatalysts [137]. In this solid solution, both $\mathrm{Zn} 3 \mathrm{~d}$ and $\mathrm{N} 2 \mathrm{p}$ electrons were present in the VBM, and $\mathrm{p}-\mathrm{d}$ repulsion occurred between them $[137,138]$, resulting in a narrower BG for this solid solution $(\sim 2.6 \mathrm{eV})$ compared with those of $\mathrm{GaN}(3.4 \mathrm{eV})$ and $\mathrm{ZnO}(3.2 \mathrm{eV}$ ) (Figure 10A). In 2017, Domen and co-workers also succeeded in creating $\mathrm{La}_{5} \mathrm{Ti}_{2} \mathrm{Cu}\left(\mathrm{S}_{1-x} \mathrm{Se}_{x}\right)_{5} \mathrm{O}_{7}\left(\mathrm{LTCS}_{1-x} \mathrm{Se}_{x}\right)$, which is a solid solution of $\mathrm{La}_{5} \mathrm{Ti}_{2} \mathrm{CuS}_{5} \mathrm{O}_{7}(\sim 1.9 \mathrm{eV})$ and $\mathrm{La}_{5} \mathrm{Ti}_{2} \mathrm{CuSe}_{5} \mathrm{O}_{7}(1.5 \mathrm{eV})$ (Figure 7 and Table 1) [132]. The absorption edge of this solid solution shifted monotonically toward longer wavelengths with increasing Se content and, at $x=1$, the solid solution absorbed light up to $820 \mathrm{~nm}$ (Figure 10B). At $x=0.2$, HER activity was observed under Vis-light irradiation of up to $720 \mathrm{~nm}$ (Figure 10B). 
(A)

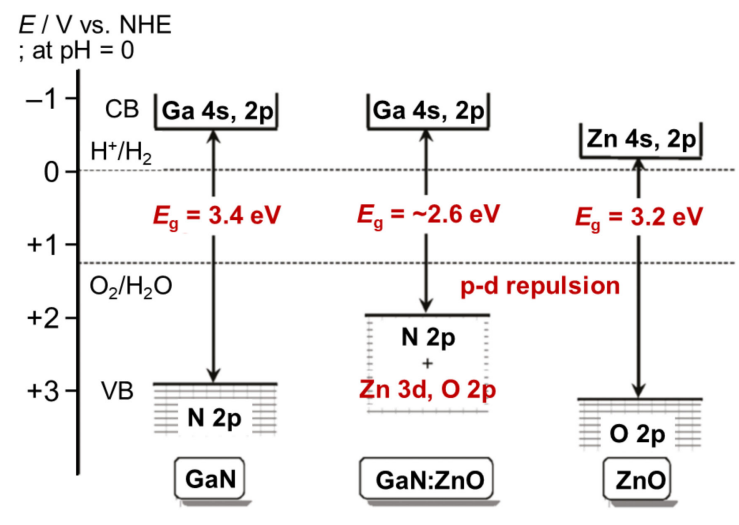

(B)

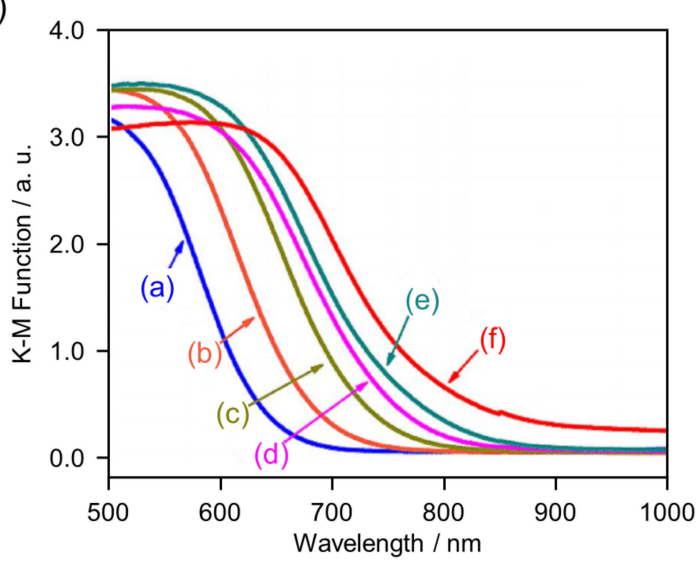

Figure 10. (A) Schematic illustration of band structures of $\mathrm{GaN}, \mathrm{ZnO}$, and their solid solution. (B) Diffuse reflectance spectra for $\mathrm{LTCS}_{1-x} \mathrm{Se}_{x} \mathrm{O}$ solid solutions as a function of Se content. Se contents: (a) 0.0, (b) 0.2, (c) 0.4, (d) 0.6, (e) 0.8, and (f) 1.0. Reproduced with permission from references [132,137]. Copyright 2010 American Chemical Society and 2017 Wiley-VCH.

\subsubsection{Shift of Band Structure by Reducing Particle Size}

In 2014, Bao and co-workers showed that reducing the size of cobalt (II) oxide (CoO) NPs to $\sim 10 \mathrm{~nm}$ shifted their band to a more suitable position for water splitting (Figure 11A). The CoO NPs with a particle size of $\sim 10 \mathrm{~nm}$ had a BG $(2.6 \mathrm{eV})$ capable of absorbing Vis light (Figure 7 and Table 1) and induced overall water splitting with a very high efficiency of $\mathrm{STH}=5 \%$ [134]. Unfortunately, $\mathrm{CoO}$ NPs have low stability, like most other Vis-light-driven photocatalysts, and the reaction was deactivated in about $1 \mathrm{~h}$. To address this problem, in 2017, Mao, Liu, Kang, and co-workers created a CoO single crystal with a submicrometer-sized octahedral structure and the active (111) face exposed (Figure 11B) [135]. In this photocatalyst, exposure of the (111) surface suppressed hydrogen peroxide $\left(\mathrm{H}_{2} \mathrm{O}_{2}\right)$ poisoning, which led to a decrease in photocatalytic activity (Figure 11C). However, even $\mathrm{CoO}$ single crystals obtained in this manner did not show sufficient stability, with thermal oxidation of $\mathrm{CoO}$ to $\mathrm{Co}_{3} \mathrm{O}_{4}$ observed. Therefore, these authors combined $\mathrm{CoO}$ single crystals with graphene, a thermal conductor, to create a highly active and stable Vis-light-driven water-splitting photocatalyst (Figure 11D). Furthermore, in 2017, Kang, Liu, Huang, and co-workers succeeded in improving stability during the degradation reaction of antimicrobial agents by forming a heterojunction of CoO NPs, a p-type semiconductor photocatalyst, and graphitic carbon nitride $\left(\mathrm{g}-\mathrm{C}_{3} \mathrm{~N}_{4}\right)$, an n-type semiconductor photocatalyst, to promote charge separation [139]. Lu, Shi, and co-workers have shown that such high stability due to heterojunctions can also be induced using $\mathrm{BiVO}_{4}$, an n-type semiconductor photocatalyst [140]. Unfortunately, there have been no reports on the water-splitting activity of such heterojunction photocatalysts using CoO NPs and n-type semiconductor photocatalysts, but water splitting is expected to be studied on such heterojunction photocatalysts in the future. 
(A)

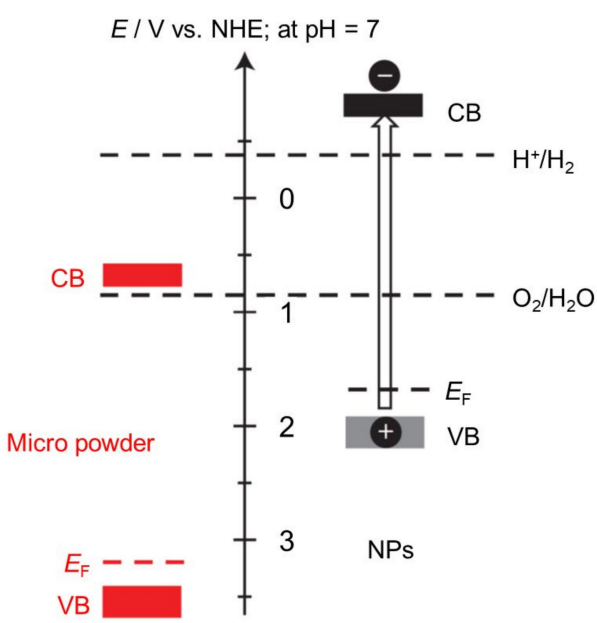

(C)

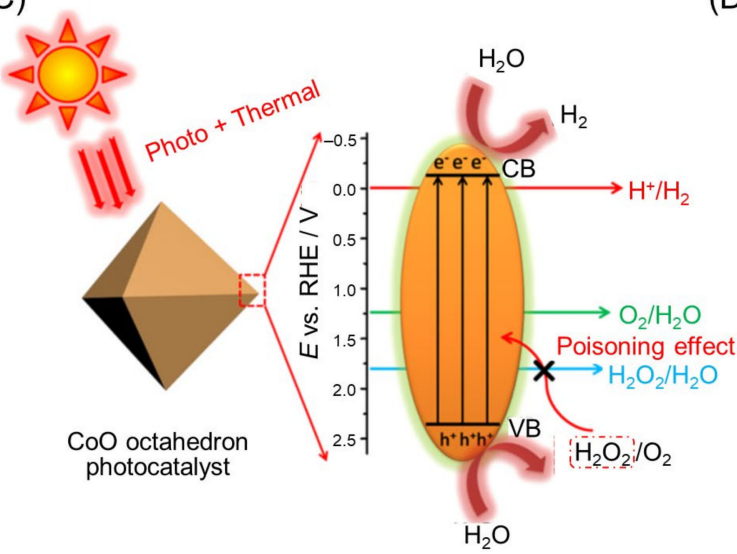

(B)

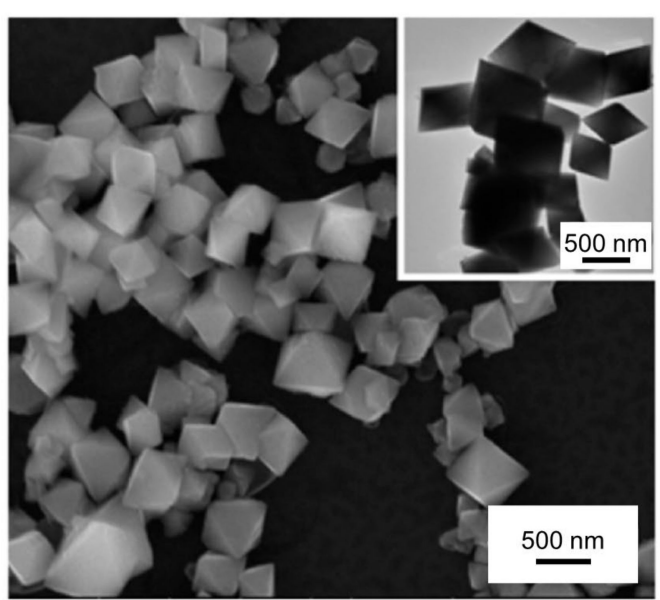

(D)

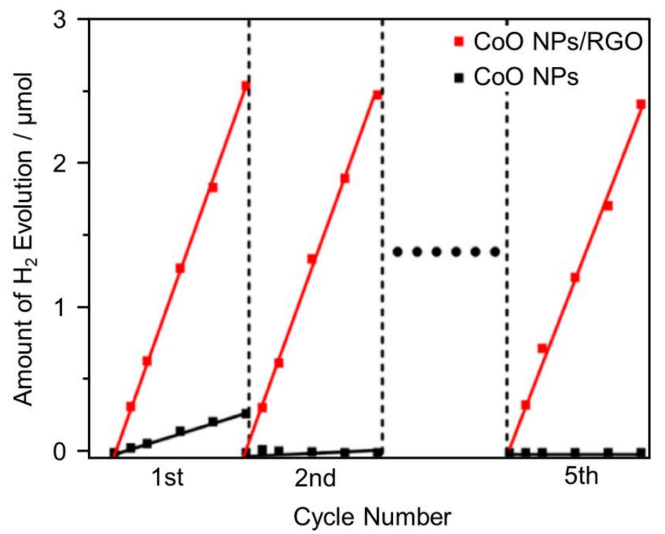

Figure 11. (A) Band positions of $\mathrm{CoO}$ micropowders and NPs. (B) Scanning electron microscopy (SEM) image of $\mathrm{CoO}$ octahedra (inset, transmission electron microscope (TEM) image). (C) Mechanism of overall water splitting over $\mathrm{CoO}$ octahedron photocatalyst. (D) Cycle stability of $\mathrm{H}_{2}$ evolution from pure water under Vis-light irradiation $(\lambda>400 \mathrm{~nm})$ using $\mathrm{CoO} /$ reduced grapheneoxide (RGO) composite and $\mathrm{CoO}$ octahedra (each cycle is $24 \mathrm{~h}$ ). Reproduced with permission from references [134,135]. Copyright 2014 Springer Nature Limited and 2017 American Chemical Society.

\subsection{Use of Other Semiconductor Materials}

In recent years, several studies have been reported on the fabrication of Vis-lightdriven water-splitting photocatalysts based on semiconductors other than metal oxides. In this section, we describe some representative photocatalysts reported in such studies. The BG energy, type, and loading amount of appropriate cocatalysts, possible reactions (OWSR, HER, or OER; Figure 4), activity, and references for semiconductor photocatalysts described in this section are summarized in Figure 7 and Table 2 [51,141-154]. 
Table 2. BG, Effective cocatalysts, possible reaction, and efficiency of Vis-light-driven water-splitting photocatalysts introduced in Section 2.2.

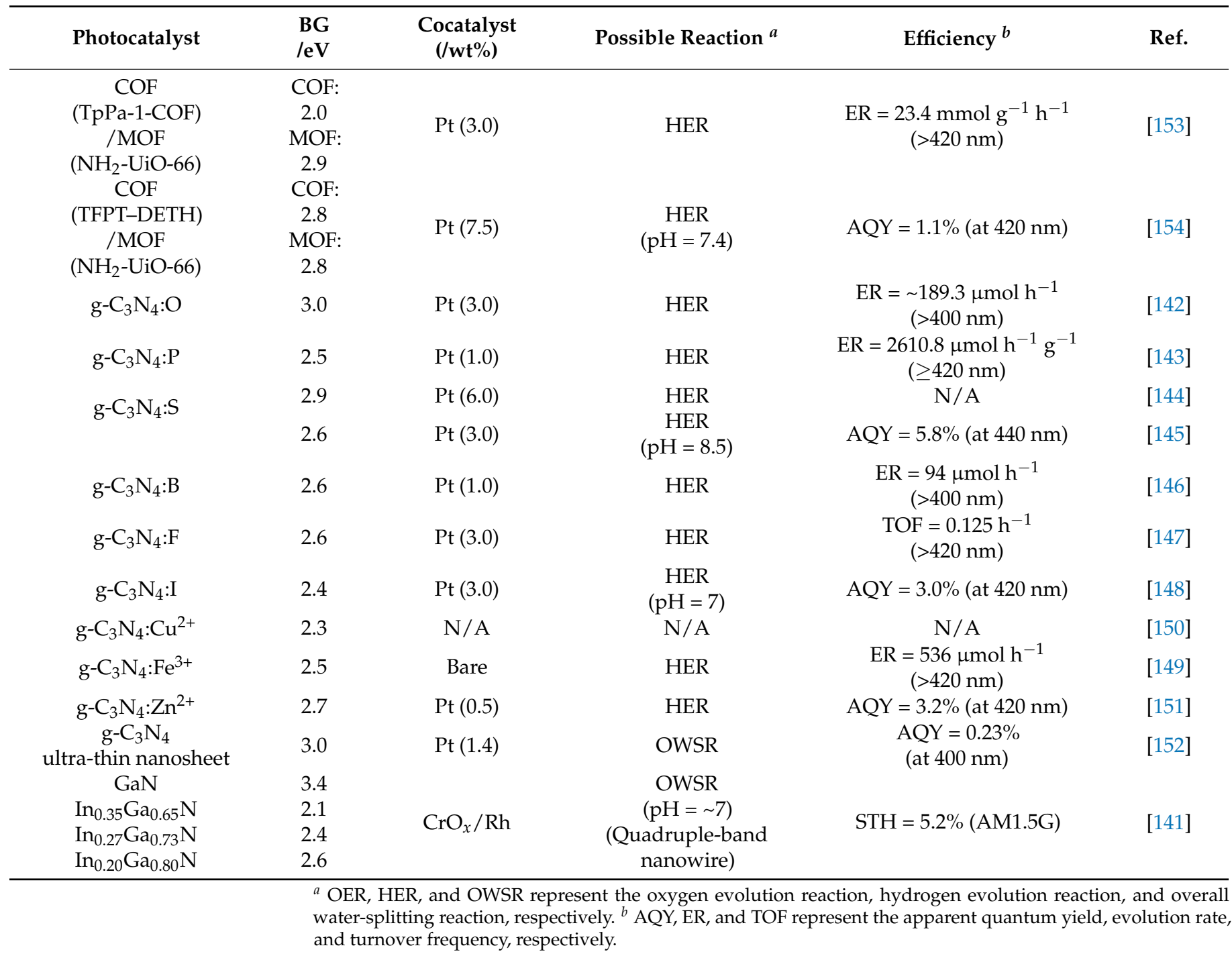

\subsubsection{Connection of Indium Gallium Nitride with Different BGs}

For indium gallium nitride ( $\mathrm{InGaN}$ ), the BG varies continuously from the UV to nearinfrared regions depending on the composition of In and Ga. In 2018, Vayssieres, Mi, and co-workers developed a photocatalyst with a dual-band structure (Figure 12A) by combining this material with $\mathrm{GaN}$ (3.4 eV) [155]. In 2019, these authors also succeeded in creating a nanowire photocatalyst with a quadruple-band structure (Figure 12B) composed of $\operatorname{In}_{0.35} \mathrm{Ga}_{0.65} \mathrm{~N}(2.1 \mathrm{eV}), \mathrm{In}_{0.27} \mathrm{Ga}_{0.73} \mathrm{~N}(2.4 \mathrm{eV}), \mathrm{In}_{0.20} \mathrm{Ga}_{0.80} \mathrm{~N}(2.6 \mathrm{eV})$, and $\mathrm{GaN}$ $(3.4 \mathrm{eV})$ [141]. The multiband InGaN nanowires were directly grown on a non-planar silicon wafer, had a large surface area, and absorbed light with high efficiency. Furthermore, the combination of four types of photocatalysts with different BGs allowed this photocatalyst to absorb sunlight across almost the entire Vis-light spectrum (Figure 12C). The obtained photocatalyst maintained its water-splitting activity for a long time under Vis-light irradiation (Figure 12D). 
(A)

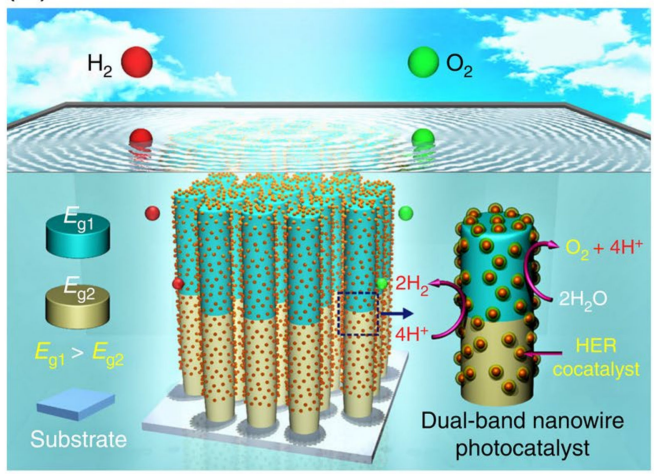

(C)

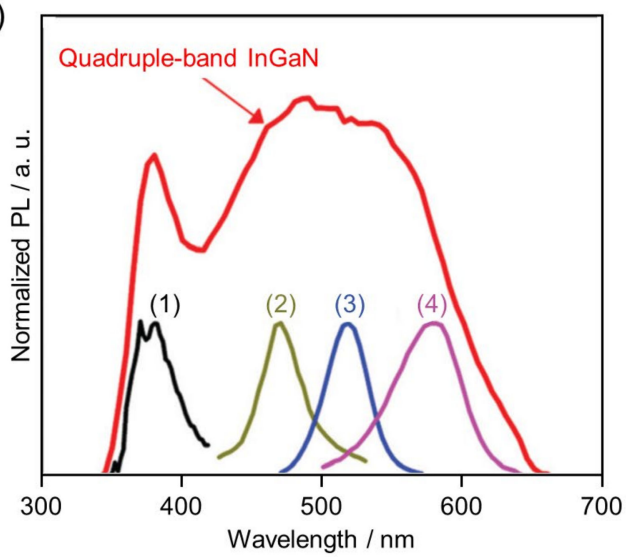

(B)

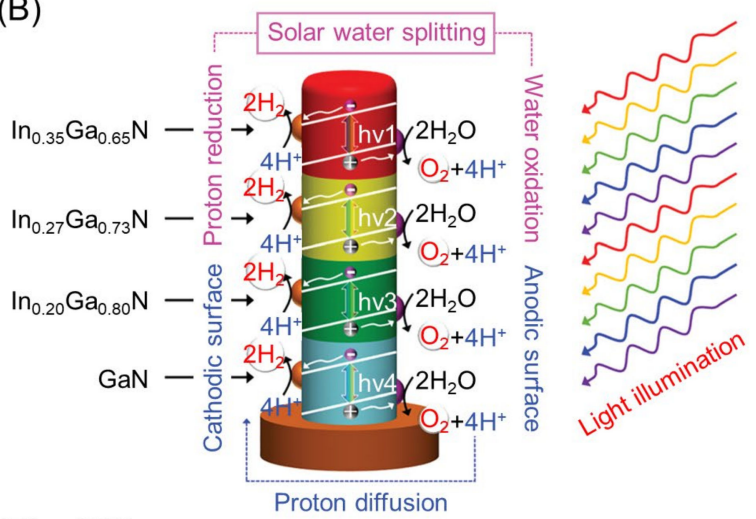

(D)

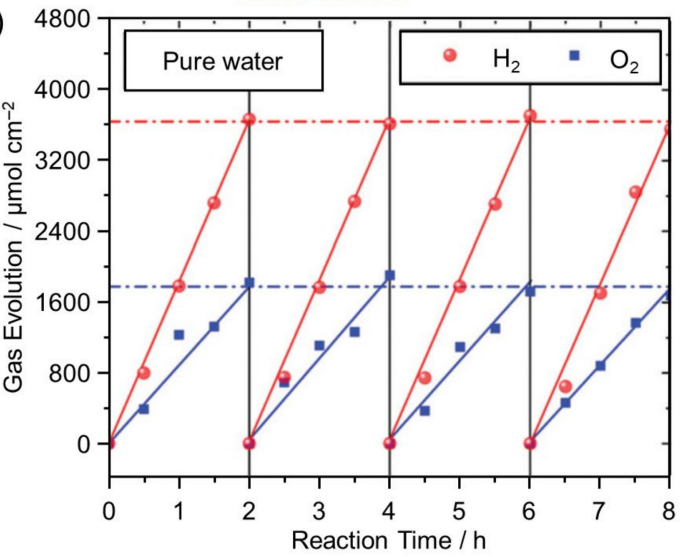

Figure 12. (A) Schematic illustration of wafer-level unassisted photocatalytic overall water splitting on dual-band nanowire arrays, which are vertically aligned on a planar substrate and decorated with cocatalysts for HER. Both OER and HER occur on the radial non-polar surfaces of each layer. (B) Schematic illustration of the overall water-splitting process occurring on the quadruple-band nanowires under light irradiation. (C) Room-temperature photoluminescence (PL) spectrum of the quadruple-band InGaN nanowires, together with those of single-band nanowires including (1) ptype GaN, (2) p- $\mathrm{In}_{0.20} \mathrm{Ga}_{0.80} \mathrm{~N}$, (3) $\mathrm{p}-\mathrm{In}_{0.27} \mathrm{Ga}_{0.73} \mathrm{~N}$, and (4) p-In $\mathrm{In}_{0.35} \mathrm{Ga}_{0.65} \mathrm{~N}$. (D) Cycle stability of $\mathrm{H}_{2}$ and $\mathrm{O}_{2}$ evolution from pure water using quadruple-band InGaN nanowire arrays with a $300 \mathrm{~W}$ xenon (Xe) lamp equipped with an AM1.5G filter (each cycle is $2 \mathrm{~h}$ ). Reproduced with permission from references [141,155]. Copyright 2018 Springer Nature Limited and 2019 The Royal Society of Chemistry.

\subsubsection{Use of Metal-Free Semiconductors $\mathrm{g}-\mathrm{C}_{3} \mathrm{~N}_{4}$}

As mentioned above, the constituent elements of Vis-light-driven water-splitting photocatalysts reported to date often include rare metals (such as $\mathrm{Ti}, \mathrm{V}, \mathrm{Cr}$, manganese $(\mathrm{Mn}), \mathrm{Co}, \mathrm{Ga}, \mathrm{Se}, \mathrm{Sr}, \mathrm{Zr}, \mathrm{Nb}, \mathrm{Mo}, \mathrm{Sb}, \mathrm{Ta}$, and $\mathrm{Bi}$ ) and rare earths (such as La) $[94-98,100-103,108-110,112,114,116-120,128,129,131,132,134,141]$. Despite their limited reserves, these metals are used in various industries, such as semiconductors and automobiles. Therefore, if Vis-light-driven water-splitting photocatalysts were implemented in society, the cost of these raw materials would soar, and the price of $\mathrm{H}_{2}$ might continue to increase. Accordingly, the development of Vis-light-driven water-splitting photocatalysts that are free of rare metals and rare earths is also currently in progress.

g- $\mathrm{C}_{3} \mathrm{~N}_{4}$ is a metal-free organic semiconductor photocatalyst that can be readily synthesized by thermal polymerization of $\mathrm{N}$-containing precursors, such as urea, melamine, and cyanamide (Figure 13A) [156-159]. g- $\mathrm{C}_{3} \mathrm{~N}_{4}$ can also be synthesized at low cost because these precursors are earth-abundant. Furthermore, for $\mathrm{g}-\mathrm{C}_{3} \mathrm{~N}_{4}(\mathrm{BG}=2.7-2.9 \mathrm{eV}$; Figure 7 and Table 2), the CBM is composed of $C p_{z}$ orbitals, and the VBM is composed of $N$ $\mathrm{p}_{z}$ orbitals, with band positions suitable for water splitting [160]. Accordingly, g- $\mathrm{C}_{3} \mathrm{~N}_{4}$ 
has attracted significant attention as a next-generation Vis-light-driven water-splitting photocatalyst [161-173].

(A)

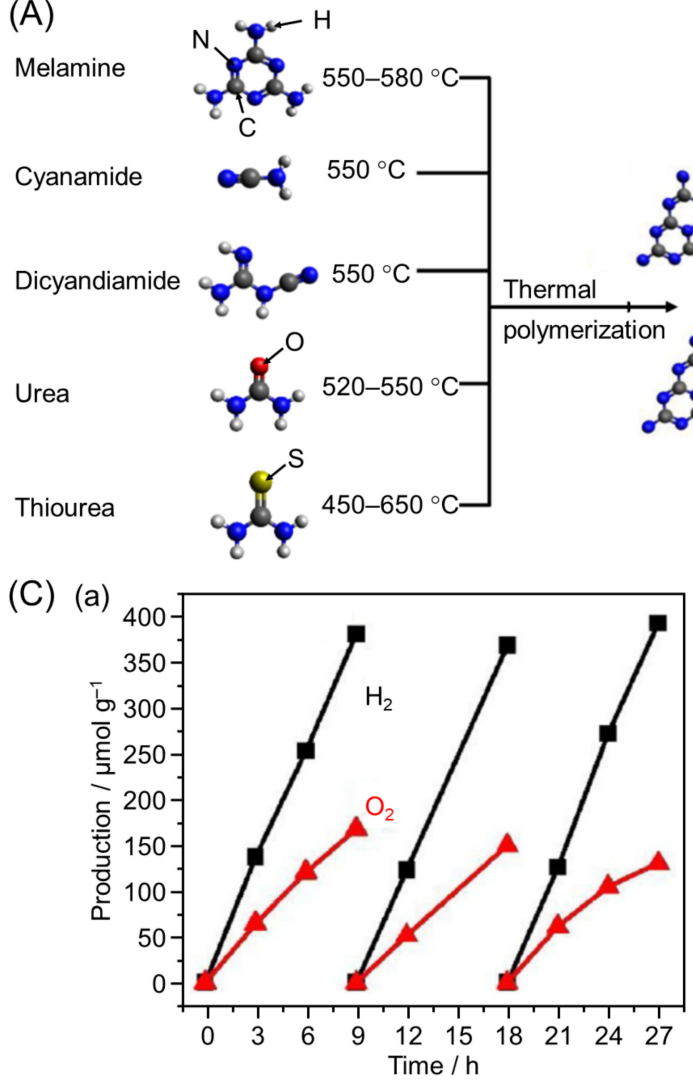

(B)

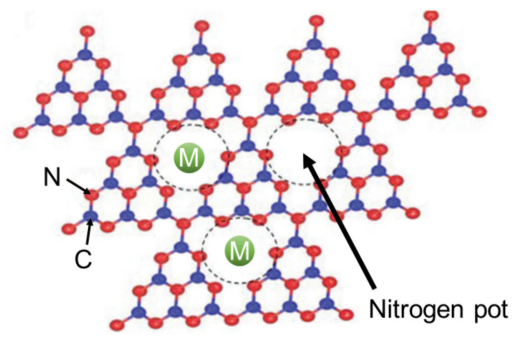

g- $\mathrm{C}_{3} \mathrm{~N}_{4}$

(b)

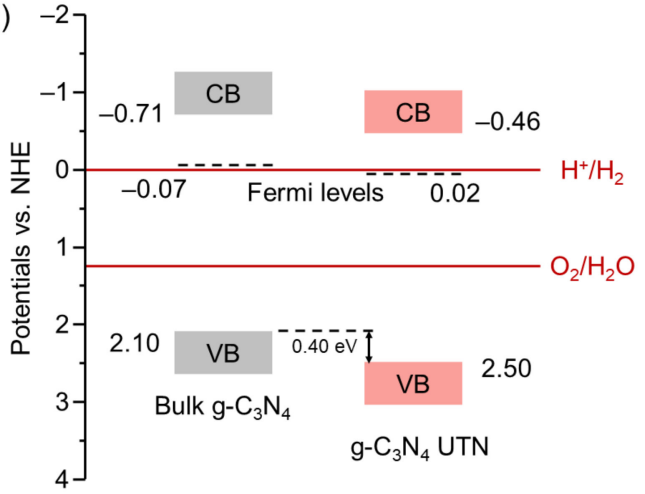

Figure 13. (A) Schematic illustration of the synthesis process of $g-\mathrm{C}_{3} \mathrm{~N}_{4}$ by thermal polymerization of different precursors, such as melamine, cyanamide, dicyanamide, urea, and thiourea. Black, blue, white, red, and yellow balls denote $\mathrm{C}, \mathrm{N}, \mathrm{H}, \mathrm{O}$, and $\mathrm{S}$ atoms, respectively. (B) Schematic diagram of a perfect g- $\mathrm{C}_{3} \mathrm{~N}_{4}$ sheet constructed from tri-s-triazine units. Red and blue balls denote $\mathrm{N}$ and $\mathrm{C}$ atoms, respectively. $\bigcirc$ indicates the nitrogen pot filled with six nitrogen lone-pair electrons, which are potentially ideal sites for metal (M) inclusion. (C) (a) The photocatalytic overall water splitting of $\mathrm{g}-\mathrm{C}_{3} \mathrm{~N}_{4}$ ultra-thin nanosheets with $\sim 1.4 \mathrm{wt} \%$ of Pt atoms as cocatalyst from pure water under Vis-light irradiation $\left(\lambda>400 \mathrm{~nm}\right.$ ). (b) The band edge positions of bulk $\mathrm{g}-\mathrm{C}_{3} \mathrm{~N}_{4}$ and $\mathrm{g}-\mathrm{C}_{3} \mathrm{~N}_{4}$ ultra-thin nanosheets. Reproduced with permission from references [152,156,171]. Copyright 2016 American Chemical Society, 2014 The Royal Society of Chemistry, and 2021 Elsevier.

The electronic structure of $\mathrm{g}-\mathrm{C}_{3} \mathrm{~N}_{4}$ can also be changed by the substitution or introduction of different atoms. Many examples of modifying the electronic structure of g- $\mathrm{C}_{3} \mathrm{~N}_{4}$ by substitution with $\mathrm{O}, \mathrm{C}, \mathrm{P}, \mathrm{S}$, boron (B), I, and $\mathrm{F}$ have been reported (Figure 7 and Table 2) [142-148,170]. For example, substituting some of the $\mathrm{N}$ in $\mathrm{g}_{-} \mathrm{C}_{3} \mathrm{~N}_{4}$ with $\mathrm{S}$ shifts both the VBM and CBM to the negative side, resulting in improved HER activity of the photocatalyst [144]. In addition to these substitutions, incorporating metal cations, such as ferric ion $\left(\mathrm{Fe}^{3+}\right), \mathrm{Cu}^{2+}, \mathrm{Zn}^{2+}$, and nickel ion $\left(\mathrm{Ni}^{3+}\right)$, into the nitrogen pot of $\mathrm{g}-\mathrm{C}_{3} \mathrm{~N}_{4}$ (Figure 13B) has been shown to change the optical and electronic properties (such as reduced BG, accelerated charge transfer, and extended charge carrier lifetime) [149-151,171].

In addition, several studies have shown that OWSR can be achieved by changing the morphology of $\mathrm{g}-\mathrm{C}_{3} \mathrm{~N}_{4}$ [172,173]. For example, in $2021 \mathrm{Bao}, \mathrm{Pu}$, and Wang successfully exfoliated g- $\mathrm{C}_{3} \mathrm{~N}_{4}$ by irradiating bulk g- $\mathrm{C}_{3} \mathrm{~N}_{4}$ with a femtosecond pulsed laser and thereby synthesized g- $\mathrm{C}_{3} \mathrm{~N}_{4}$ ultra-thin nanosheets (UTN) [152]. The deposition of platinum (Pt) single atoms on $\mathrm{g}-\mathrm{C}_{3} \mathrm{~N}_{4} \mathrm{UTN}$ resulted in OWSR with a $\mathrm{H}_{2}$ production rate of $42.6 \mu \mathrm{mol} \mathrm{g}{ }^{-1} \mathrm{~h}^{-1}$ and an $\mathrm{O}_{2}$ production rate of $18.7 \mu \mathrm{mol} \mathrm{g}^{-1} \mathrm{~h}^{-1}$ (Figure $13 \mathrm{C}(\mathrm{a})$ ). They 
attributed this phenomenon to the formation of $\mathrm{C} \equiv \mathrm{N}$ defects caused by laser stripping, which induced the two following things: (1) aiding the loading of Pt single atoms and thereby increasing the number of active sites, and (2) downshifting the CBM and VBM to promote the OER (Figure 13C(b)).

Connection of Covalent Organic Frameworks with Metal-Organic Frameworks or Covalent Organic Frameworks

Covalent organic frameworks (COFs) and metal-organic frameworks (MOFs) are crystalline and porous materials that consist of pure organic molecules and metals bonded by covalent bonds or coordination bonds. They are used in various fields, such as gas storage, catalysis, and sensing. COFs have long-range ordered structure, excellent surface area, and adjustable BG and have recently attracted much attention as water-splitting photocatalysts [174]. Most two-dimensional COFs reported to date form layered structures through $\pi-\pi$ stacking, which allows them to transport charge carriers not only within but also between covalent sheets [175-177]. Furthermore, most COFs (especially Schiff-based $\mathrm{COFs}$ ) have colors ranging from orange to dark red and provide excellent light harvesting in the Vis-light range. However, COFs not only exhibit low hydrophilicity and stability but also easily cause the recombination of photoexcited carriers. Therefore, the watersplitting activity of COFs has generally been lower than those of metal oxide (sulfide) Vis-light-driven water-splitting photocatalysts.

In 2018, Lan and co-workers reported the development of a hybrid photocatalyst of a COF (TpPa-1-COF) and a MOF ( $\left.\mathrm{NH}_{2}-\mathrm{UiO}-66\right)$ [153]. The hybrid photocatalyst had a band structure suitable for charge separation, and the COF and MOF were strongly connected by covalent bonds (Figure 14A). In these hybrid photocatalysts, the transfer of excited electrons occurred efficiently (Figure 14B), resulting in high HER activity (Table 2).

(A)

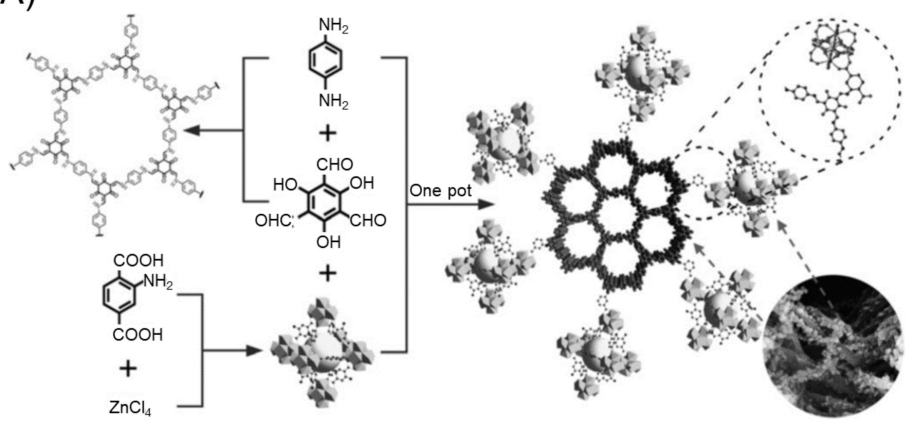

(B)

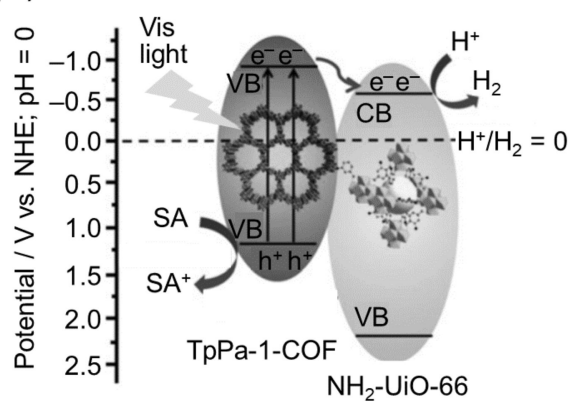

Figure 14. (A) Schematic illustration of the synthesis of the hybrid material composed of TpPa-1-COF (COF; upper compounds) and $\mathrm{NH}_{2}-\mathrm{UiO}-66$ (MOF; lower compounds). (B) Schematic of mechanism of HER over the hybrid material (TpPa-1-COF: $\mathrm{NH}_{2}-\mathrm{UiO}-66=4: 6$ (optimal ratio)). SA represents the sodium ascorbate. Reproduced with permission from reference [153]. Copyright 2018 Wiley-VCH.

Other groups have also been working on the development of such hybrid photocatalysts. For example, using a MOF $\left(\mathrm{NH}_{2}-\mathrm{UiO}-66\right)$ and a $\mathrm{COF}$ (1,3,5-tris-(4-formylphenyl) triazine (TFPT)-2,5-diethoxybenzene-1,4-dicarbohydrazide (DETH)), Jiang and co-workers successfully created octahedral core-shell hetero-framework photocatalysts (TFPT-DETH/ $\mathrm{NH}_{2}-\mathrm{UiO}-66$ ) [154]. These photocatalysts were formed by epitaxial growth of a TFPT-DETH shell on a $\mathrm{NH}_{2}-\mathrm{UiO}-66$ core (Figure $\left.15 \mathrm{~A}\right)$. The authors obtained a series of TFPT-DETH/ $\mathrm{NH}_{2}$-UiO-66 samples (TFPT-DETH/ $\mathrm{NH}_{2}-\mathrm{UiO}-66(n)(n=1,2,4$, or 6$)$ ) with different shell thicknesses by varying the amounts of TFPT and DETH. As shown in Figure 15B, the HER rate increased with increasing TFPT shell thickness, reaching a maximum at DETH/ $\mathrm{NH}_{2}-\mathrm{UiO}-66$ (4), and then steadily decreased. These phenomena were due to some of the bulk material, including the MOF core, not being able to absorb sufficient light when the COF shell was too thick, which reduced the amount of photogenerated excitons. Furthermore, for organic semiconductors, a thicker shell results in a 
faster exciton recombination rate inside the photocatalyst owing to the shorter diffusion length and lower mobility of excitons. Owing to these factors, the photocatalytic activity of $\mathrm{DETH} / \mathrm{NH}_{2}-\mathrm{UiO}-66(n)$ samples gave the highest HER rate when the thickness of the COF shell was at the optimum value $(n=4)$. The DETH/ $\mathrm{NH}_{2}-\mathrm{UiO}-66(4)$ photocatalyst showed excellent HER activity with an apparent quantum yield (AQY) of $1.11 \%$ (at $420 \mathrm{~nm}$ ). The authors suggested that synergistic effects in the hetero-framework were responsible for this high AQY (Figure 15C).

(A)

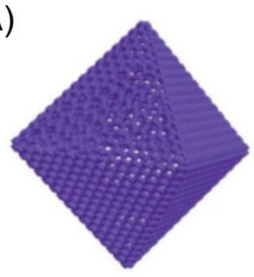

$\mathrm{NH}_{2}-\mathrm{UiO}-66$

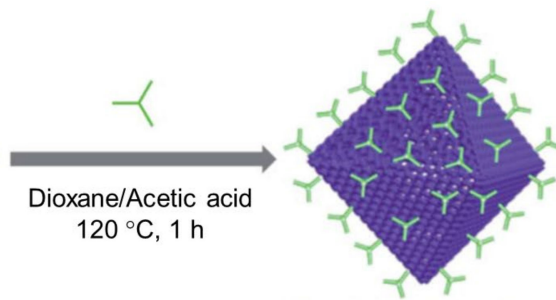

TFPT/ $\mathrm{NH}_{2}-\mathrm{UiO}-66$

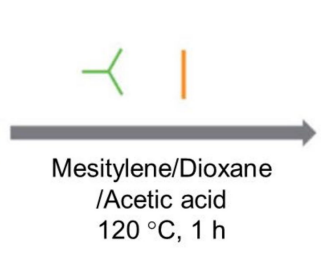

(C)

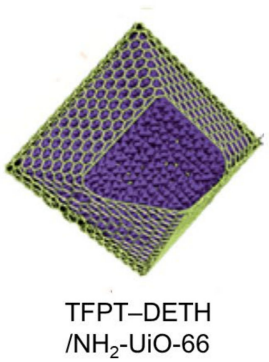

$/ \mathrm{NH}_{2}-\mathrm{UiO}-66$
(B)

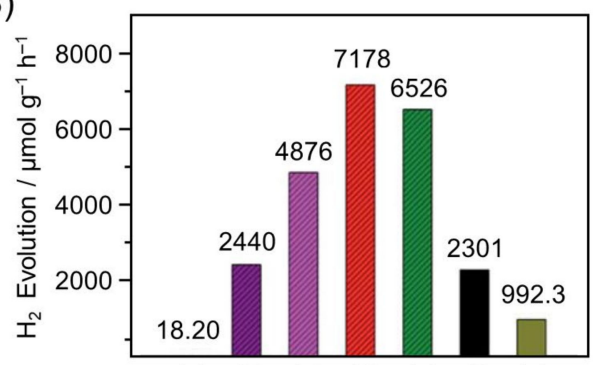

(a) (b) (c) (d) (e) (f) (g)

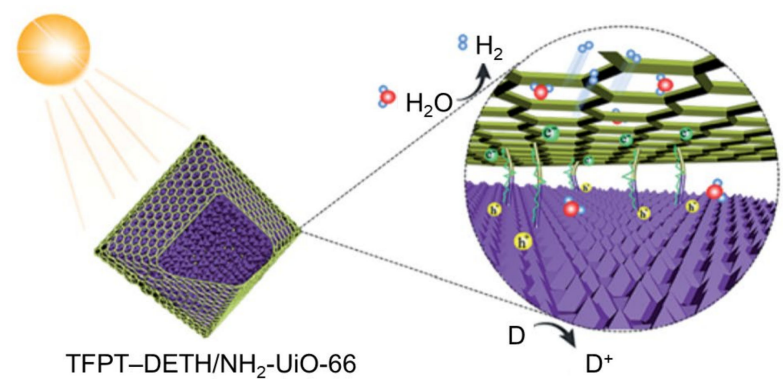

Figure 15. (A) Schematic illustration of the synthetic process of TFPT-DETH/NH $2-\mathrm{UiO}-66$ core-shell hetero-frameworks. (B) Corresponding HER rates of photocatalysts using phosphate-buffered saline buffer solution $(\mathrm{pH}=7.4)$ containing $100 \mathrm{mg}$ of sodium ascorbate as a sacrificial electron donor, and Pt NPs (7.5 wt\%) as a cocatalyst, under Vis-light irradiation $\left(\lambda \geq 420 \mathrm{~nm}, \mathrm{Xe}\right.$ lamp): (a) $\mathrm{NH}_{2}$ UiO-66, (b-e) TFPT-DETH/NH 2 -UiO-66 ( $n$ ) samples $(n=1,2,4$, or 6), (f) TFPT-DETH, and (g) physical mixture of TFPT-DETH and $\mathrm{NH}_{2}-\mathrm{UiO}-66$ (TFPT-DETH:NH illustration of photocatalytic $\mathrm{H}_{2}$ evolution over the TFPT-DETH/ $\mathrm{NH}_{2}-\mathrm{UiO}-66(n)$ hetero-framework under Vis-light irradiation. In the figure, D denotes sodium ascorbate. Reproduced with permission from reference [154]. Copyright 2019 The Royal Society of Chemistry.

\section{Control of Cocatalysts}

As described in Section 2, the development of Vis-light-driven water-splitting photocatalysts has seen rapid progress in recent years (Figure 7, Tables 1 and 2). However, water splitting was only achieved using a semiconductor photocatalyst alone in a few reported cases. This is largely due to two factors, as follows: (i) Most of the photoexcited charges recombine in a short time and relax energetically, producing heat (non-radiative deactivation) and luminescence (radiative deactivation); and (ii) the surface of the semiconductor photocatalyst has few active sites for reaction. Therefore, for the photocatalytic reaction to proceed with a high quantum yield (QY), electrons and holes must be spatially separated and transferred to active sites on the surface before recombination.

Cocatalysts promote the transfer of excitons generated in the photocatalyst and act as active sites to lower the activation energy of the HER and OER. When selecting a suitable HER cocatalyst, the volcano plot (Figure 16A) obtained from the results of Langmuir-type adsorption of $\mathrm{H}_{2}$ is often used [178-180]. This volcano plot shows that the catalyst surface with the optimal binding energy to reactants and products has the highest catalytic activity. Therefore, noble metals, such as $\mathrm{Pt}$ [181-184] and Rh [104,185], for which the $\mathrm{H}_{2}$ adsorption free energy $\left(\Delta \mathrm{G}_{\mathrm{H}^{*}}\right)$ is near 0 , are often used as elements in HER cocatalysts. Recently, HER cocatalysts without precious metal elements (such as $\mathrm{MoS}_{2}$ [186-189], NiS [41,190-195], 
CoMoS [196-199], $\mathrm{Ni}_{2} \mathrm{P}$ [200-204], and FeP [205-207]) have also been developed. Meanwhile, metal oxides (sulfides), such as $\mathrm{CoO}_{x}$ [208-210], $\mathrm{RuO}_{2}$ [211-213], $\mathrm{IrO}_{2}$ [120,214], and PdS [215,216], are often used as OER cocatalysts, based on the volcano plot shown in Figure 16B [217].
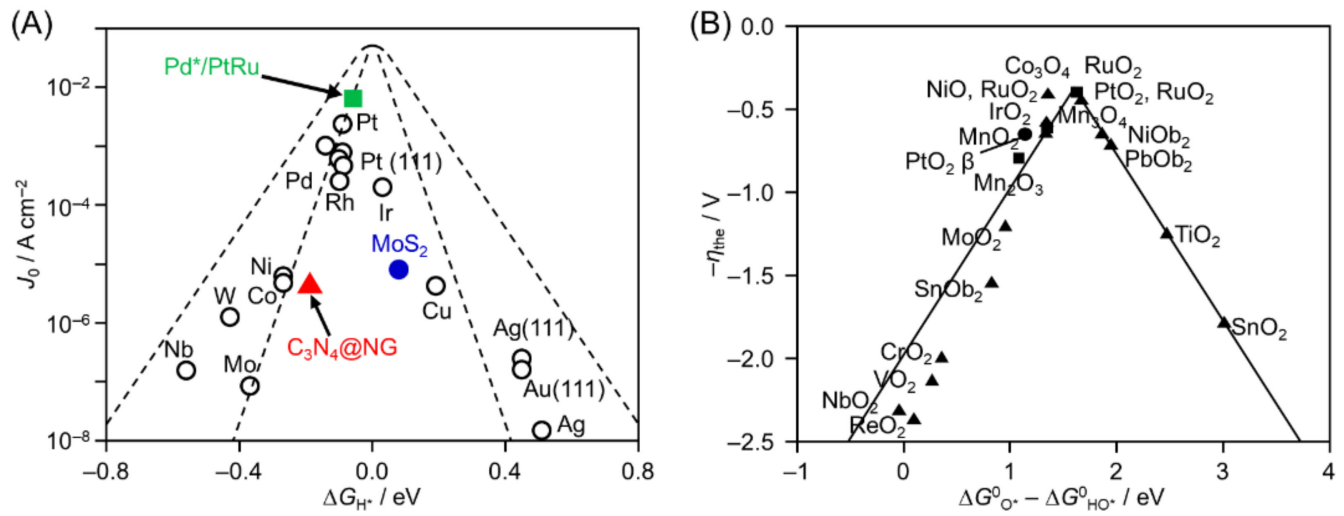

Figure 16. (A) The HER $j_{0}$ (exchange current density) values as a function of the free energy from the adsorption of $\mathrm{H}_{2}\left(\Delta \mathrm{G}_{\mathrm{H}^{*}}\right)$ for the surfaces of various metals, alloys, and nonmetallic materials. (B) $-\eta_{\text {the }}$ (negative values of theoretical overpotential) values for the OER as a function of $\left(\Delta \mathrm{G}^{0} \mathrm{O}^{*}-\Delta \mathrm{G}^{0} \mathrm{HO}^{*}\right)$. Reproduced with permission from references $[178,217]$. Copyright 2014 Springer Nature Limited and 2014 Wiley-VCH.

Thus, each reaction consists of the adsorption of reactants on the cocatalyst surface, reaction on the cocatalyst surface, and desorption from the cocatalyst surface, and these processes are largely related to the adsorption and desorption energy at the cocatalyst surface. Therefore, these properties need to be considered when improving the functionality of the photocatalyst by modifying the cocatalyst. This section discusses six approaches to improving such cocatalysts, as follows: (i) particle-size control (Section 3.1; Figure 17A) [73-75,77,218]; (ii) chemical composition control (Section 3.2; Figure 17B) [76,219,220]; (iii) morphology control (Section 3.3; Figure 17C) [221-224]; (iv) interface structure control (Section 3.4; Figure 17D) [225]; (v) surface-structure control (Section 3.5; Figure 17E) [77,226-241]; and (vi) charge-state control (Section 3.6; Figure 17F) [242,243]. For the semiconductor photocatalysts described in this section, the appropriate cocatalysts, possible reactions (OWSR, HER, or OER; Figure 4), activities, and references are summarized in Table 3 [74-77,218-225,234,241-243]. 
(B) Chemical composition
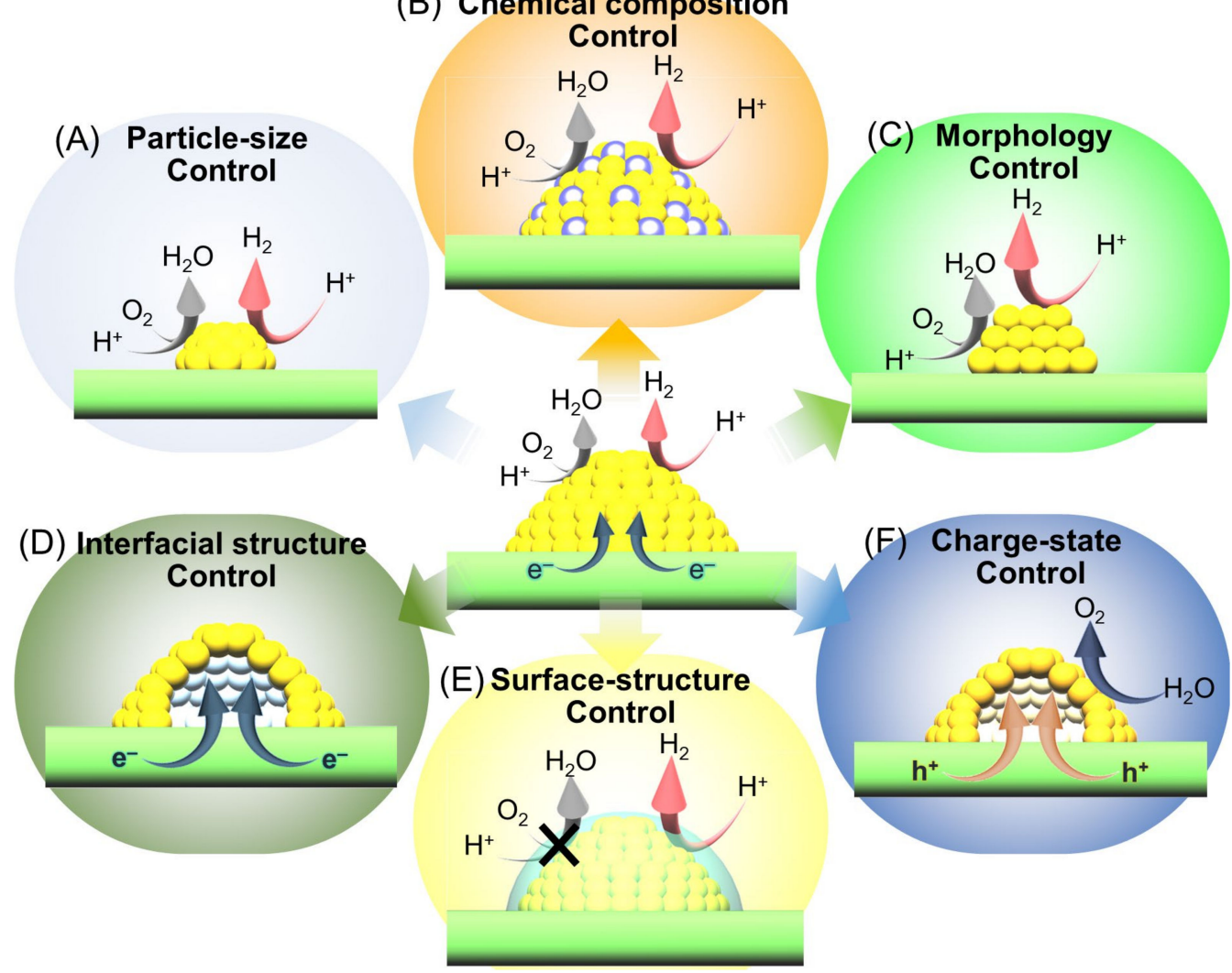

Figure 17. Schematic of photocatalyst functionalization by controlling cocatalyst properties: (A) particle size (Section 3.1), (B) chemical composition (Section 3.2), (C) morphology (Section 3.3), (D) interfacial structure (Section 3.4), (E) surface structure (Section 3.5), and (F) charge state (Section 3.6).

Table 3. Effective cocatalysts, possible reaction, and efficiency of water-splitting photocatalysts (Vis-light-driven or UV-light-driven) introduced in Section 3.

\begin{tabular}{|c|c|c|c|c|c|}
\hline Photocatalyst & $\begin{array}{c}\text { Cocatalyst } \\
(/ \mathbf{w t} \%)^{a}\end{array}$ & $\begin{array}{l}\text { Size } \\
/ \mathrm{nm}\end{array}$ & $\begin{array}{c}\text { Possible } \\
\text { Reaction } b\end{array}$ & Efficiency $^{c}$ & Ref. \\
\hline \multirow[t]{8}{*}{$\mathrm{BaLa}_{4} \mathrm{Ti}_{4} \mathrm{O}_{15}$} & $\mathrm{Au}$ NPs (0.5) & $12.3 \pm 3.7$ & OWSR & $\begin{array}{c}\mathrm{ER}\left(\mathrm{H}_{2}\right)=\sim 60 \mu \mathrm{mol} \mathrm{h} \mathrm{h}^{-1} \\
(400 \mathrm{~W} \mathrm{Hg} \mathrm{lamp)}\end{array}$ & [74] \\
\hline & $\mathrm{Au}_{10} \mathrm{NC}(0.1)$ & $0.9 \pm 0.2$ & OWSR & $\begin{array}{c}\mathrm{ER}\left(\mathrm{H}_{2}\right)=\sim 260 \mu \mathrm{mol} \mathrm{h}^{-1} \\
(400 \mathrm{~W} \mathrm{Hg} \mathrm{lamp})\end{array}$ & [74] \\
\hline & $\mathrm{Au}_{15} \mathrm{NC}(0.1)$ & $1.0 \pm 0.2$ & OWSR & $\begin{array}{c}\text { ER }\left(\mathrm{H}_{2}\right)=\sim 220 \mu \mathrm{mol} \mathrm{h}^{-1} \\
(400 \mathrm{~W} \mathrm{Hg} \mathrm{lamp})\end{array}$ & [74] \\
\hline & $\mathrm{Au}_{18} \mathrm{NC}(0.1)$ & $1.1 \pm 0.2$ & OWSR & $\begin{array}{c}\mathrm{ER}\left(\mathrm{H}_{2}\right)=\sim 210 \mu \mathrm{mol} \mathrm{h} \mathrm{h}^{-1} \\
(400 \mathrm{~W} \mathrm{Hg} \text { lamp })\end{array}$ & [74] \\
\hline & $\mathrm{Au}_{25} \mathrm{NC}(0.1)$ & $1.2 \pm 0.3$ & OWSR & $\begin{array}{c}\mathrm{ER}\left(\mathrm{H}_{2}\right)=\sim 180 \mu \mathrm{mol} \mathrm{h}^{-1} \\
(400 \mathrm{~W} \mathrm{Hg} \mathrm{lamp})\end{array}$ & [74] \\
\hline & $\mathrm{Au}_{39} \mathrm{NC}(0.1)$ & $1.5 \pm 0.3$ & OWSR & $\begin{array}{c}\operatorname{ER}\left(\mathrm{H}_{2}\right)=\sim 170 \mu \mathrm{mol} \mathrm{h}{ }^{-1} \\
(400 \mathrm{~W} \mathrm{Hg} \mathrm{lamp})\end{array}$ & [74] \\
\hline & $\begin{array}{c}\mathrm{Cr}_{2} \mathrm{O}_{3} / \mathrm{Au}_{25} \mathrm{NC}(\mathrm{Cr}: 0.5 \\
\mathrm{Au}: 0.1)\end{array}$ & $1.1 \pm 0.3$ & OWSR & $\begin{array}{c}\text { ER }\left(\mathrm{H}_{2}\right)=3032 \mu \mathrm{mol} \mathrm{h}^{-1} \\
(400 \mathrm{~W} \mathrm{Hg} \mathrm{lamp)}\end{array}$ & [75] \\
\hline & $\begin{array}{c}\mathrm{Au}_{24} \mathrm{Pd} \mathrm{NC} \\
(\mathrm{Au}: 0.1)\end{array}$ & $1.1 \pm 0.2$ & OWSR & $\begin{array}{c}\text { ER }\left(\mathrm{H}_{2}\right)=\sim 100 \mu \mathrm{mol} \mathrm{h}^{-1} \\
(400 \mathrm{~W} \mathrm{Hg} \mathrm{lamp)}\end{array}$ & [76] \\
\hline
\end{tabular}


Table 3. Cont.

\begin{tabular}{|c|c|c|c|c|c|}
\hline Photocatalyst & $\begin{array}{l}\text { Cocatalyst } \\
(/ \mathbf{w t} \%)^{a}\end{array}$ & $\begin{array}{l}\text { Size } \\
/ \mathrm{nm}\end{array}$ & $\begin{array}{l}\text { Possible } \\
\text { Reaction } b\end{array}$ & Efficiency $^{c}$ & Ref. \\
\hline & $\begin{array}{c}\mathrm{Au}_{24} \mathrm{Pt} \mathrm{NC} \\
(\mathrm{Au}: 0.1)\end{array}$ & $1.1 \pm 0.2$ & OWSR & $\begin{array}{c}\mathrm{ER}\left(\mathrm{H}_{2}\right)=\sim 125 \mu \mathrm{mol} \mathrm{h} \mathrm{h}^{-1} \\
(400 \mathrm{~W} \mathrm{Hg} \text { lamp })\end{array}$ & [76] \\
\hline & $\begin{array}{c}\mathrm{Cr}_{2} \mathrm{O}_{3} / \\
\mathrm{Au}_{24} \mathrm{Pt} \mathrm{NC} \\
(\mathrm{Cr}: 0.3, \mathrm{Au}: 0.1)\end{array}$ & $1.3 \pm 0.3$ & OWSR & $\begin{array}{c}\text { ER }\left(\mathrm{H}_{2}\right)=\sim 2500 \mu \mathrm{mol} \mathrm{h} \mathrm{h}^{-1} \\
(400 \mathrm{~W} \mathrm{Hg} \mathrm{lamp)}\end{array}$ & [76] \\
\hline & $\begin{array}{c}\mathrm{Rh}_{2-x} \mathrm{Cr}_{x} \mathrm{O}_{3} \mathrm{NPs} \\
\text { (Rh: } 0.09 \\
\text { Cr: } 0.1 \text { ) }\end{array}$ & $1.2 \pm 0.2$ & OWSR & $\begin{array}{c}\mathrm{ER}\left(\mathrm{H}_{2}\right)=9.9 \mathrm{mmol} \mathrm{h}^{-1} \\
(400 \mathrm{~W} \mathrm{Hg} \text { lamp }) \\
\text { AQY }=16 \%(\text { at } 270 \mathrm{~nm})\end{array}$ & [77] \\
\hline $\mathrm{BaTaO}_{2} \mathrm{~N}$ & $\begin{array}{c}\mathrm{Pt}(\mathrm{IMP}) / \mathrm{Pt}(\mathrm{PD}) \mathrm{NPs}(\mathrm{Pt}(\mathrm{IMP}): \\
0.1, \mathrm{Pt}(\mathrm{PD}): 0.2)\end{array}$ & $\mathrm{N} / \mathrm{A}$ & HER & $\begin{array}{l}\mathrm{AQY}=6.8 \pm 0.5 \% \\
\quad(\text { at } 420 \mathrm{~nm})\end{array}$ & [225] \\
\hline \multirow[t]{5}{*}{$\mathrm{CdS}$} & cubic-Pd NPs & 10.0 & HER & $\begin{array}{c}\mathrm{ER}=814 \mu \mathrm{mol} \mathrm{h}^{-1} \\
(>420 \mathrm{~nm})\end{array}$ & [223] \\
\hline & $\begin{array}{l}\text { octahedral- } \\
\text { Pd NPs }\end{array}$ & 7.7 & HER & $\begin{array}{c}\mathrm{ER}=591 \mu \mathrm{mol} \mathrm{h}^{-1} \\
(>420 \mathrm{~nm})\end{array}$ & [223] \\
\hline & spherical-Pd NPs & $\mathrm{N} / \mathrm{A}$ & HER & $\begin{array}{c}\mathrm{ER}=489 \mu \mathrm{mol} \mathrm{h}^{-1} \\
(>420 \mathrm{~nm})\end{array}$ & [223] \\
\hline & cubic-PtPd NPs (0.5) & 7.3 & HER & $\mathrm{AQY}=54.0 \%($ at $420 \mathrm{~nm})$ & [224] \\
\hline & octahedral-PtPd NPs (0.5) & 4.7 & HER & $\mathrm{AQY}=36.6 \%($ at $420 \mathrm{~nm})$ & [224] \\
\hline \multirow[t]{5}{*}{$\mathrm{g}-\mathrm{C}_{3} \mathrm{~N}_{4}$} & $\mathrm{CoAl}_{2} \mathrm{O}_{4} \mathrm{NPs}(3.0)$ & $5-20$ & $\begin{array}{c}\text { OER } \\
(\mathrm{pH}= \\
8.0-8.5)\end{array}$ & $\mathrm{AQY}=0.2 \%($ at $420 \mathrm{~nm})$ & [242] \\
\hline & $\mathrm{Pt}_{0.5} \mathrm{Co}_{0.5}$ NPs (1.0) & $3-5$ & HER & $\begin{array}{c}\mathrm{ER}=960 \mu \mathrm{mol} \mathrm{g}^{-1} \mathrm{~h}^{-1} \\
(\geq 400 \mathrm{~nm})\end{array}$ & [220] \\
\hline & $\begin{array}{c}\text { cubic-Pt NPs } \\
(0.89)\end{array}$ & 10.5 & HER & $\begin{array}{c}\mathrm{ER}=20.4 \mu \mathrm{mol} \mathrm{h}^{-1} \\
(>400 \mathrm{~nm})\end{array}$ & [221] \\
\hline & octahedral-Pt NPs (0.90) & 11.0 & HER & $\begin{aligned} & \mathrm{ER}= 24.7 \mu \mathrm{mol} \mathrm{h}^{-1} \\
&(>400 \mathrm{~nm})\end{aligned}$ & [221] \\
\hline & spherical-Pt NPs (0.87) & 9.4 & HER & $\begin{array}{c}\mathrm{ER}=229.4 \mu \mathrm{mol} \mathrm{h}^{-1} \\
(>400 \mathrm{~nm})\end{array}$ & [221] \\
\hline \multirow[t]{3}{*}{ GaN:ZnO } & $\begin{array}{l}\mathrm{Cr}_{2} \mathrm{O}_{3} / \mathrm{Rh} \mathrm{NPs} \\
\text { (Cr: } 1.5 \text {, Rh: } 0.3 \text { ) }\end{array}$ & $1.5 \pm 0.3$ & $\begin{array}{c}\text { HER } \\
(\mathrm{pH}=4.5)\end{array}$ & $\begin{aligned} \mathrm{ER}= & \sim 620 \mu \mathrm{mol} \mathrm{h}-1 \\
& (>400 \mathrm{~nm})\end{aligned}$ & [218] \\
\hline & $\begin{array}{l}\mathrm{Cr}_{2} \mathrm{O}_{3} / \mathrm{Rh} \text { NPs } \\
\text { (Cr: } 1.5, \text { Rh: } 0.3 \text { ) }\end{array}$ & $3.8 \pm 0.8$ & $\begin{array}{c}\text { HER } \\
(\mathrm{pH}=4.5)\end{array}$ & $\begin{aligned} \mathrm{ER}= & \sim 430 \mu \mathrm{mol} \mathrm{h}^{-1} \\
& (>400 \mathrm{~nm})\end{aligned}$ & [218] \\
\hline & $\begin{array}{l}\mathrm{Cr}_{2} \mathrm{O}_{3} / \mathrm{Rh} \mathrm{NPs} \\
\text { (Cr: 1.5, Rh: 0.3) }\end{array}$ & $6.6 \pm 1.1$ & $\begin{array}{c}\text { HER } \\
(\mathrm{pH}=4.5)\end{array}$ & $\begin{aligned} \mathrm{ER}= & \sim 260 \mu \mathrm{mol} \mathrm{h}{ }^{-1} \\
& (>400 \mathrm{~nm})\end{aligned}$ & [218] \\
\hline $\mathrm{LaMg}_{1 / 3} \mathrm{Ta}_{2 / 3} \mathrm{O}_{2} \mathrm{~N}$ & $\begin{array}{c}\mathrm{TiOXH} \\
\text { /SiOXHRhCrO } \mathrm{NPs}_{x} \\
\text { (Cr: 0.5, Rh: 0.5) }\end{array}$ & $\mathrm{N} / \mathrm{A}$ & OWSR & $\begin{array}{c}\mathrm{AQY}=0.03 \% \\
(\text { at } 440 \pm 30 \mathrm{~nm})\end{array}$ & [234] \\
\hline $\mathrm{SrTiO}_{3}$ & $\begin{array}{c}\mathrm{Cu}_{0.95} \mathrm{Pt}_{0.05} \mathrm{NPs} \\
(1.5 \mathrm{~mol} \%)\end{array}$ & $\sim 3.0$ & HER & $\begin{array}{l}\mathrm{ER}=369.4 \mu \mathrm{mol} \mathrm{h}^{-1} \\
\quad(500 \mathrm{~W} \mathrm{Hg} \mathrm{lamp})\end{array}$ & [219] \\
\hline $\mathrm{SrTiO}_{3}: \mathrm{Al}$ & $\begin{array}{l}\mathrm{RhZrO} \text { NPs } \\
(\mathrm{Zr}: 0.5, \mathrm{Rh}: 0.1) \\
-\mathrm{CoO}_{x}(0.1)\end{array}$ & $\mathrm{N} / \mathrm{A}$ & OWSR & $\begin{array}{l}\mathrm{AQY}=33 \pm 4.0 \% \\
\quad(\text { at } 365 \mathrm{~nm})\end{array}$ & [241] \\
\hline $\begin{array}{c}\mathrm{TiO}_{2} \\
\text { (anatase) }\end{array}$ & $\begin{array}{l}\text { cubic-Pd NPs } \\
(2.7)\end{array}$ & $\sim 14$ & $\begin{array}{c}\text { HER } \\
(\mathrm{pH}=3.0)\end{array}$ & $\begin{aligned} \mathrm{ER}=\underset{(>420 \mathrm{~nm})}{\sim} & 6.0 \mu \mathrm{mol} \mathrm{g}^{-1} \mathrm{~h}^{-1}\end{aligned}$ & [222] \\
\hline $\begin{array}{c}\mathrm{TiO}_{2} \\
\text { (anatase) }\end{array}$ & tetrahedral-Pd NPs (2.8) & $\sim 14$ & $\begin{array}{c}\text { HER } \\
(\mathrm{pH}=3.0)\end{array}$ & $\begin{aligned} \mathrm{ER}= & \sim 10 \mu \mathrm{mol} \mathrm{g}^{-1} \mathrm{~h}^{-1} \\
& (>420 \mathrm{~nm})\end{aligned}$ & [222] \\
\hline $\mathrm{TaON}$ & $\begin{array}{c}\mathrm{CoO}_{x} \text { NPs } \\
\quad(0.15)\end{array}$ & $\sim 2.5$ & $\begin{array}{c}\text { OER } \\
(\mathrm{pH}=\sim 8.5)\end{array}$ & $\begin{aligned} \mathrm{AQY} & =21.2 \% \\
(\text { at } 420 & \pm 15 \mathrm{~nm})\end{aligned}$ & [243] \\
\hline
\end{tabular}

${ }^{a}$ For example, $\mathrm{Pt}(\mathrm{IMP})$ and $\mathrm{Pt}(\mathrm{PD}) \mathrm{NPs}$ represent Pt NPs loaded by impregnation and photodeposition, respectively. ${ }^{b}$ OER, HER, and OWSR represent the oxygen evolution reaction, hydrogen evolution reaction, and overall water-splitting reaction, respectively. ${ }^{c} \mathrm{AQY}, \mathrm{ER}$, and $\mathrm{Hg}$ represent the apparent quantum yield, evolution rate, and mercury, respectively. 


\subsection{Particle Size Control}

The size of cocatalyst NPs has a significant effect on the photocatalytic activity. For example, Teranishi and co-workers synthesized monodisperse Rh NPs of different sizes $(1.6 \pm 0.5,2.7 \pm 0.3$, or $5.1 \pm 0.5 \mathrm{~nm})$ using a polyol synthesis method, and then this HER cocatalyst was loaded on $\mathrm{GaN}: \mathrm{ZnO}$ ( $\mathrm{Rh} \mathrm{NPs} / \mathrm{GaN}: \mathrm{ZnO}$ ) by removing the ligands via calcination [218]. Transmission electron microscopy (TEM) images (Figure 18A) showed that the Rh NPs cocatalyst was slightly aggregated on $\mathrm{GaN}: \mathrm{ZnO}$ but still highly monodisperse $(1.5 \pm 0.3,3.8 \pm 0.8$, or $6.6 \pm 1.1 \mathrm{~nm})$. $\mathrm{A} \mathrm{Cr}_{2} \mathrm{O}_{3}$ shell (see Section 3.5.1) was then formed on the Rh NPs $\left(\mathrm{Cr}_{2} \mathrm{O}_{3} / \mathrm{Rh} \mathrm{NPs} / \mathrm{GaN}: \mathrm{ZnO}\right)$, which acted as a reverse-reaction suppressor layer. The water-splitting activity of $\mathrm{Cr}_{2} \mathrm{O}_{3} / \mathrm{Rh}$ NPs $/ \mathrm{GaN}: \mathrm{ZnO}$ was enhanced with a reduction in size of the Rh NPs cocatalyst (Figure 18B and Table 3). These size effects were attributed to an increase in the proportion of surface atoms (HER active sites) in the Rh NPs cocatalyst, and enhanced charge separation as the Rh NPs cocatalyst decreased in size. Such an enhancement of the water-splitting activity caused by a reduction in size of the cocatalyst has also been observed in our study on gold ( $\mathrm{Au}$ )-NCs or $\mathrm{Rh}_{2-x} \mathrm{Cr}_{x} \mathrm{O}_{3}-\mathrm{NCs}-\mathrm{loaded}$ $\mathrm{BaLa}_{4} \mathrm{Ti}_{4} \mathrm{O}_{15}$ photocatalysts (UV-light-driven photocatalysts; Figure 19) [73-75,77].

(A)
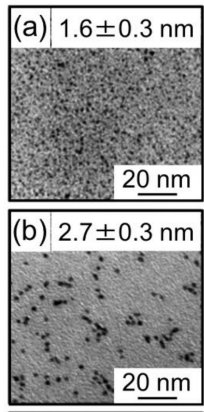

(c) $5.1 \pm 0.5 \mathrm{~nm}$

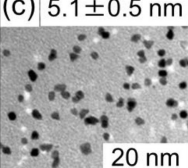

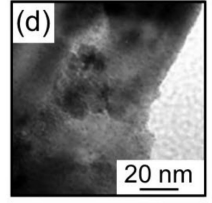
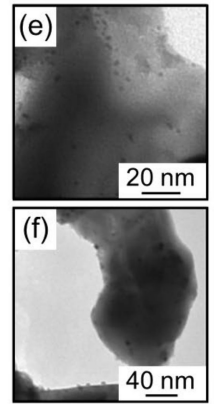
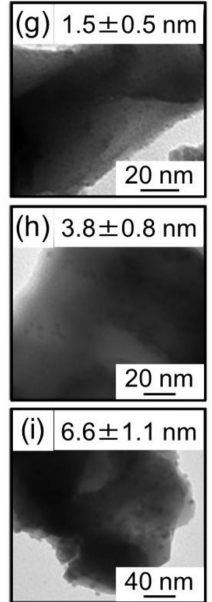

(B)

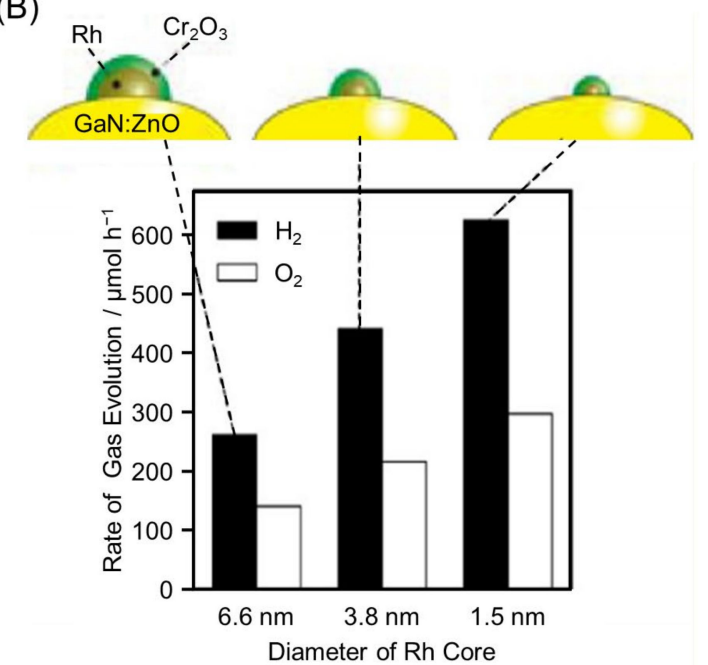

Figure 18. (A) TEM images of three types of Rh NPs: $(\mathbf{a}-\mathbf{c})$ as-synthesized, $(\mathbf{d}-\mathbf{f})$ adsorbed on $\mathrm{GaN}: \mathrm{ZnO}$, and (g-i) loaded on $\mathrm{GaN}: \mathrm{ZnO}$ after calcination. (B) Initial rates of $\mathrm{H}_{2}$ and $\mathrm{O}_{2}$ evolution over $\mathrm{GaN}: \mathrm{ZnO}$ loaded with different-sized $\mathrm{Cr}_{2} \mathrm{O}_{3} / \mathrm{Rh}$ (core-shell) NPs. Reaction conditions: catalyst, $0.15 \mathrm{~g} ; \mathrm{H}_{2} \mathrm{SO}_{4}$ aq. ( $\mathrm{pH} 4.5$ ), $400 \mathrm{~mL}$; light source, high-pressure $\mathrm{Hg}$ lamp (450 W) through $\mathrm{NaNO}_{2}$ aq filter to cut UV light; reaction vessel, Pyrex inner-irradiation type. Reproduced with permission from reference [218]. Copyright 2013 American Chemical Society.

\subsection{Chemical Composition Control}

Alloying enables the creation of materials with physical properties and functions different from those of single metals. In 2015, Qin, Kang, and co-workers showed that the HER activity of $\mathrm{SrTiO}_{3}$ was significantly enhanced when $\mathrm{Cu}-\mathrm{Pt}$ alloy NPs were used as the cocatalyst compared with using Cu NPs or Pt NPs as the cocatalyst [219]. Specifically, $\mathrm{Cu}_{0.95} \mathrm{Pt}_{0.05} \mathrm{NPs} / \mathrm{SrTiO}_{3}$ showed an HER rate about 2.79, 1.76 times higher than those of $\mathrm{Cu} \mathrm{NPs} / \mathrm{SrTiO}_{3}$ and Pt NPs $/ \mathrm{SrTiO}_{3}$, respectively (Figure 20 and Table 3). The authors interpretation of these results was that using $\mathrm{Cu}-\mathrm{Pt}$ alloy NPs as the cocatalyst led to high HER rates because excited electrons were efficiently transferred to the cocatalyst, which suppressed charge recombination. 
(A)

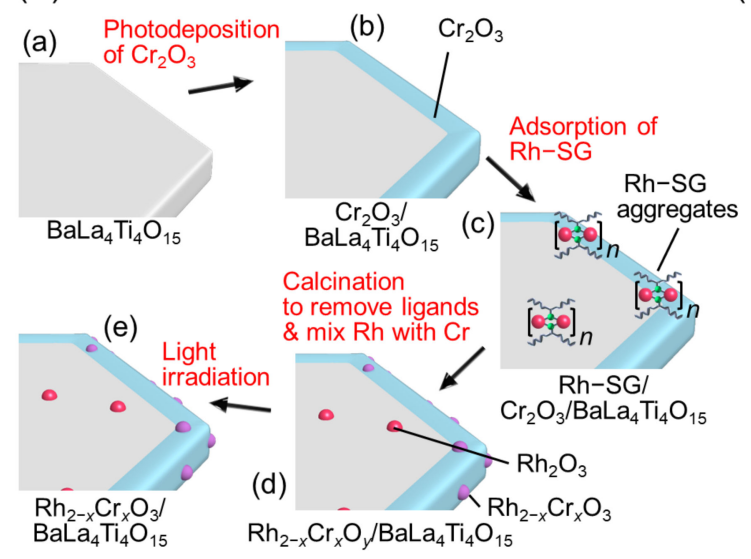

(B)

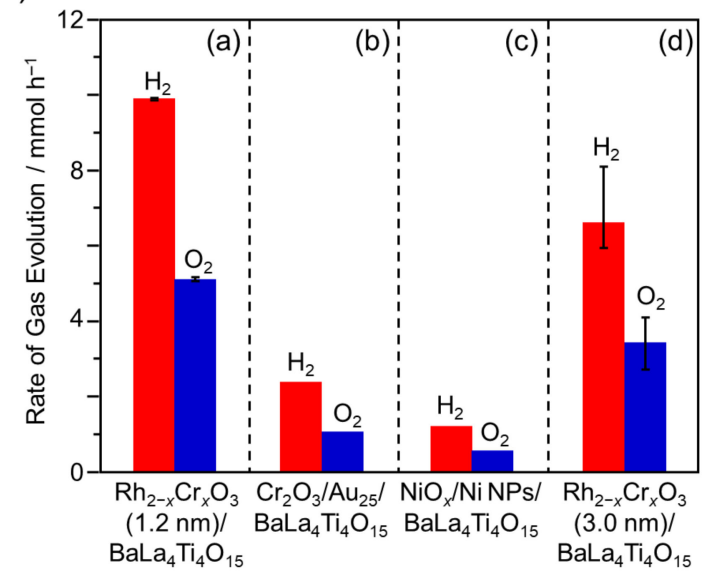

Figure 19. (A) Schematic of experimental procedure for the formation of $\mathrm{BaLa}_{4} \mathrm{Ti}_{4} \mathrm{O}_{15}$ loaded with small $\mathrm{Rh}_{2-x} \mathrm{Cr}_{x} \mathrm{O}_{3} \mathrm{NCs}(1.2 \mathrm{~nm})$ cocatalysts $\left(\mathrm{Rh}_{2-x} \mathrm{Cr}_{x} \mathrm{O}_{3}(1.2 \mathrm{~nm}) / \mathrm{BaLa}_{4} \mathrm{Ti}_{4} \mathrm{O}_{15}\right)$. (a) $\mathrm{BaLa}_{4} \mathrm{Ti}_{4} \mathrm{O}_{15}$, (b) $\mathrm{Cr}_{2} \mathrm{O}_{3} / \mathrm{BaLa}_{4} \mathrm{Ti}_{4} \mathrm{O}_{15}$, (c) Rh-SG/ $\mathrm{Cr}_{2} \mathrm{O}_{3} / \mathrm{BaLa}_{4} \mathrm{Ti}_{4} \mathrm{O}_{15}$, (d) $\mathrm{Rh}_{2-x} \mathrm{Cr}_{x} \mathrm{O}_{y}(1.2 \mathrm{~nm}) / \mathrm{BaLa}_{4} \mathrm{Ti}_{4} \mathrm{O}_{15}$, and (e) $\mathrm{Rh}_{2-x} \mathrm{Cr}_{x} \mathrm{O}_{3}(1.2 \mathrm{~nm}) / \mathrm{BaLa}_{4} \mathrm{Ti}_{4} \mathrm{O}_{15} . \mathrm{Rh}_{2-x} \mathrm{Cr}_{x} \mathrm{O}_{y}$ represents $\mathrm{Rh}_{2-x} \mathrm{Cr}_{x} \mathrm{O}_{3}$ containing highly oxidized $\mathrm{Cr}(>3+)$. (B) Comparison of $\mathrm{H}_{2}$ and $\mathrm{O}_{2}$ evolution rates from pure water using a high-pressure Hg lamp (400 W) over different photocatalysts: (a) $\mathrm{Rh}_{2-x} \mathrm{Cr}_{x} \mathrm{O}_{3}(1.2 \mathrm{~nm}) / \mathrm{BaLa}_{4} \mathrm{Ti}_{4} \mathrm{O}_{15}(0.09 \mathrm{wt} \%$ $\mathrm{Rh}$ and $0.10 \mathrm{wt} \% \mathrm{Cr}),\left(\right.$ b) $\mathrm{Cr}_{2} \mathrm{O}_{3} / \mathrm{Au}_{25} / \mathrm{BaLa}_{4} \mathrm{Ti}_{4} \mathrm{O}_{15}(0.10 \mathrm{wt} \% \mathrm{Au}$ and $0.50 \mathrm{wt} \% \mathrm{Cr}),(\mathbf{c}) \mathrm{NiO}_{x} / \mathrm{Ni}$ $\mathrm{NPs} / \mathrm{BaLa}_{4} \mathrm{Ti}_{4} \mathrm{O}_{15}(0.50 \mathrm{wt} \% \mathrm{Ni})$, and (d) $\mathrm{Rh}_{2-x} \mathrm{Cr}_{x} \mathrm{O}_{3}(3.0 \mathrm{~nm}) / \mathrm{BaLa}_{4} \mathrm{Ti}_{4} \mathrm{O}_{15}(0.10 \mathrm{wt} \% \mathrm{Rh}$ and $0.15 \mathrm{wt} \% \mathrm{Cr}$ ). In this study, $\mathrm{NiO}_{x} / \mathrm{Ni} \mathrm{NPs} / \mathrm{BaLa}_{4} \mathrm{Ti}_{4} \mathrm{O}_{15}$ and $\mathrm{Rh}_{2-x} \mathrm{Cr}_{x} \mathrm{O}_{3}(3.0 \mathrm{~nm}) / \mathrm{BaLa}_{4} \mathrm{Ti}_{4} \mathrm{O}_{15}$ were prepared using the impregnation method. Reproduced with permission from reference [77]. Copyright 2020 Wiley-VCH.

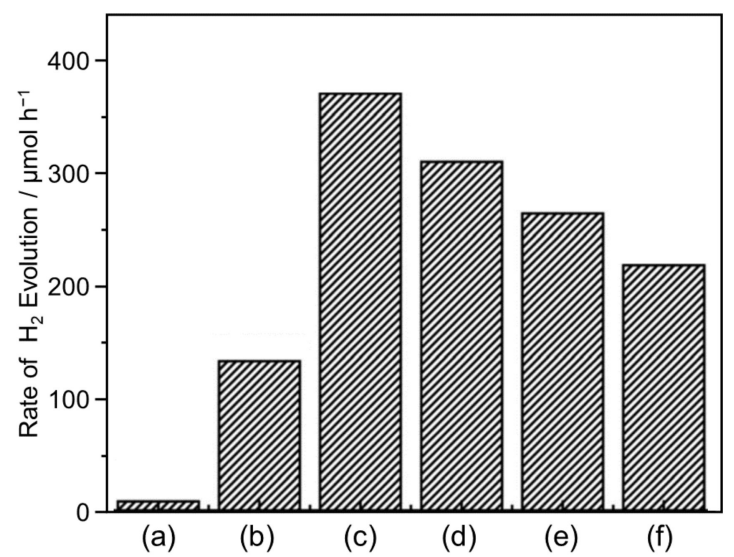

Figure 20. Photocatalytic $\mathrm{H}_{2}$ evolution from $50 \mathrm{vol} \%$ aqueous methanol solution under high-pressure Hg lamp irradiation over (a) bare $\mathrm{SrTiO}_{3}$ and (b-f) $\mathrm{Cu}_{1-x} \mathrm{Pt}_{x} / \mathrm{SrTiO}_{3}(x=$ (b) 0, (c) 0.05, (d) 0.1, (e) 0.9, or (f) 1.0) NPs. Reproduced with permission from reference [219]. Copyright 2015 The Royal Society of Chemistry.

In 2015, Ge and co-workers reported using an alloy consisting of $\mathrm{Pt}$ and $\mathrm{Co}$ as a cocatalyst [220]. In this study, $\mathrm{Pt}_{0.5} \mathrm{Co}_{0.5} \mathrm{NPs} / \mathrm{g}-\mathrm{C}_{3} \mathrm{~N}_{4}$ nanosheets were found to have HER activity 1.34 times higher than that of $\mathrm{Pt} \mathrm{NPs} / \mathrm{g}-\mathrm{C}_{3} \mathrm{~N}_{4}$ nanosheets with the same $\mathrm{Pt}$ loading (Figure 21A and Table 3). Alloying with Co was interpreted to increase the driving force for photoexcited electron transfer from the $\mathrm{CB}$ of $\mathrm{g}-\mathrm{C}_{3} \mathrm{~N}_{4}$ to the cocatalyst, resulting in enhanced HER activity (Figure 21B). However, when the Co content exceeded the Pt content, the HER activity decreased (Figure 21A). This was attributed to the reduction in HER reaction sites on the cocatalyst surface when the Co content became too large. 
(A)

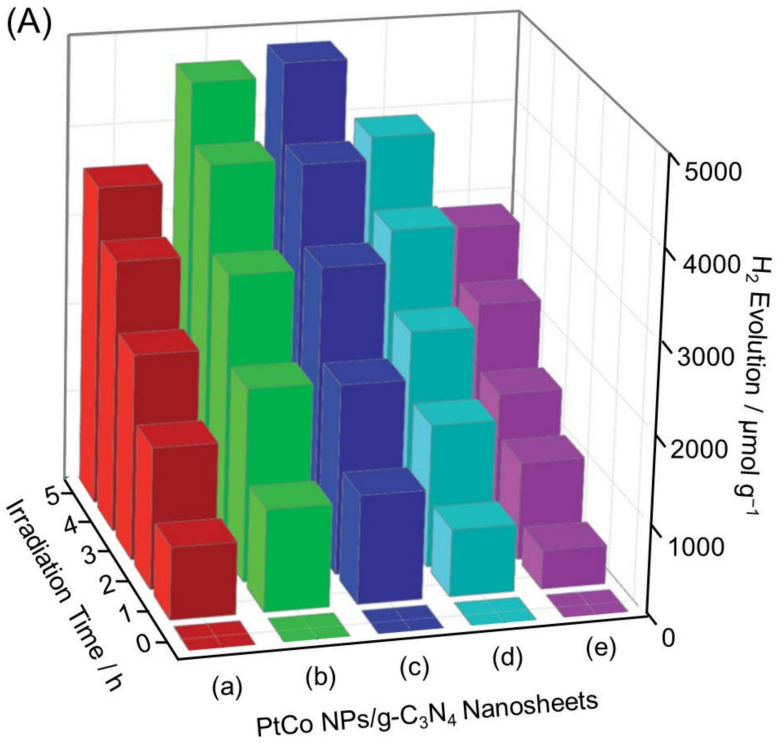

(B)

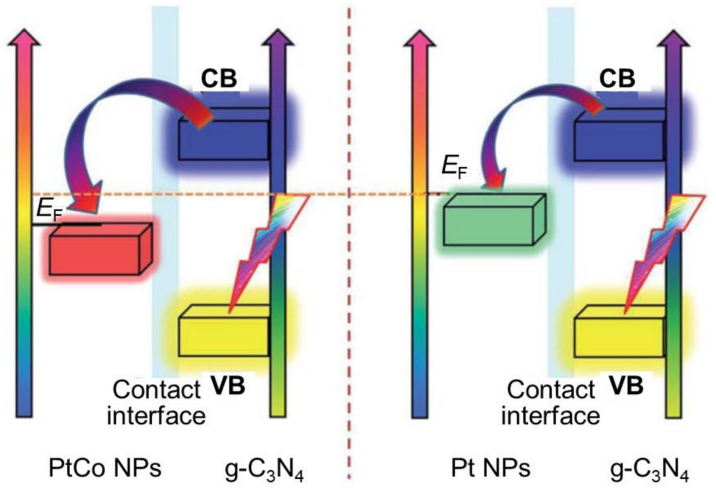

Figure 21. (A) Photocatalytic $\mathrm{H}_{2}$ evolution from aqueous solution containing $10 \mathrm{vol} \%$ of triethanolamine (sacrificial reagent) under Vis-light irradiation ( $\geq 400 \mathrm{~nm}$ ) over $\mathrm{Pt}_{1-x} \mathrm{Co}_{x} \mathrm{NPs} / \mathrm{g}-\mathrm{C}_{3} \mathrm{~N}_{4}$ nanosheets with different Pt/Co percentages at the same loading of $1.0 \mathrm{wt} \% ; x=$ (a) 0 , (b) 0.25 , (c) 0.5 , (d) 0.75 , and (e) 0.86. (B) Schematic illustration of photoexcited electron injection from $\mathrm{CB}$ of $\mathrm{g}-\mathrm{C}_{3} \mathrm{~N}_{4}$ to the PtCo NPs and Pt NPs. Reproduced with permission from reference [220]. Copyright 2015 The Royal Society of Chemistry.

As described above, alloying is extremely effective at improving the cocatalyst function. Recently, the synthesis of alloy NCs with precisely controlled chemical compositions by liquid-phase synthesis has become possible [244-283]. The use of these fine alloy NCs as precursors will allow precise control of the chemical composition of loaded metal NCs (Figure 22), which is expected to provide an improved understanding of the factors that contribute to the enhanced activity induced by alloying [86].

(A)

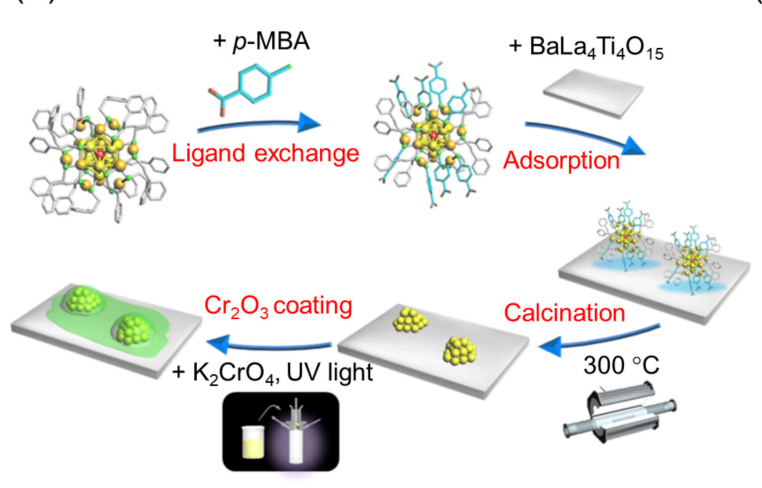

(B)

(a)

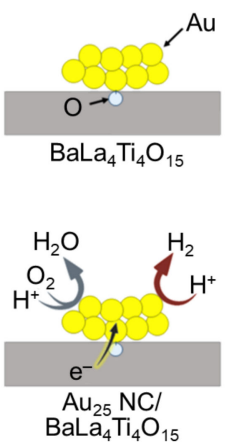

(b)

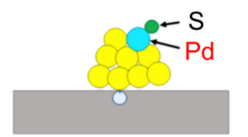

UV light $\downarrow$ (c)
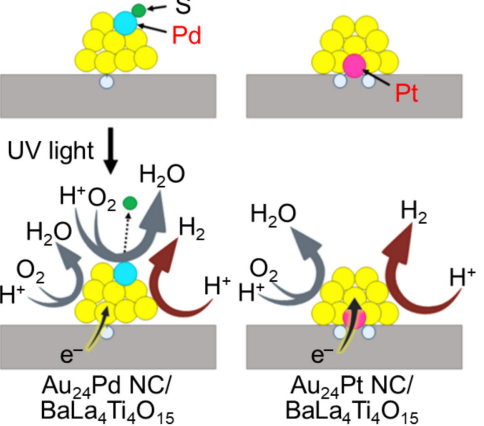

Figure 22. (A) Schematic illustration of the experimental procedure for the preparation of $\mathrm{Au}_{24} \mathrm{M}$ $\mathrm{NC} / \mathrm{BaLa}_{4} \mathrm{Ti}_{4} \mathrm{O}_{15}(\mathrm{M}=\mathrm{Au}, \mathrm{Pd}$, or Pt). $p$-MBA; 4-mercaptobenzoic acid. (B) Proposed structures of $\mathrm{Au}_{24} \mathrm{M} \mathrm{NC} / \mathrm{BaLa}_{4} \mathrm{Ti}_{4} \mathrm{O}_{15}$, where $\mathrm{M}$ is (a) $\mathrm{Au}$, (b) Pd, and (c) Pt before (above) and during (below) the water-splitting reaction. Reproduced with permission from reference [76]. Copyright 2019 American Chemical Society.

\subsection{Morphology Control}

When metal NPs are used as a cocatalyst, the water-splitting activity changes depending on the exposed crystal plane. This is due to the electronic structure and surface energy of the metal NPs' surface differing depending on the crystal plane, resulting in different 
adsorption properties with the substrate. The optimal shape and crystal planes are different depending on the metal species.

In 2016, Yu and co-workers synthesized Pt NPs with different shapes (cubic, octahedral, or spherical; $\sim 10 \mathrm{~nm}$; Figure 23A) and successfully loaded them on $\mathrm{g}-\mathrm{C}_{3} \mathrm{~N}_{4}$ [221]. Studies on the as-obtained Pt NPs $/ g-\mathrm{C}_{3} \mathrm{~N}_{4}$ photocatalysts showed that their HER activity increased in the order of cubic-Pt NPs $/ g-\mathrm{C}_{3} \mathrm{~N}_{4}<$ octahedral-Pt NPs $/ g-\mathrm{C}_{3} \mathrm{~N}_{4}<$ spherical-Pt NPs $/ g-\mathrm{C}_{3} \mathrm{~N}_{4}$ (Figure 23B and Table 3). The cubic-Pt NPs consist of six (100) planes and do not have many active sites, consisting of sharp edges and corners, which are necessary for HER evolution. In contrast, octahedral-Pt NPs are composed of eight (111) planes and have more active sites, consisting of sharp edges and corners, than cubic-Pt NPs. Meanwhile, spherical-Pt NPs are composed of a large number of (100) and (111) planes, and these structures contain many active sites consisting of sharp edges and corners. These factors were interpreted to be related to the HER activity of Pt NPs $/ \mathrm{g}-\mathrm{C}_{3} \mathrm{~N}_{4}$ with Pt NPs cocatalysts of different geometries, in the order of cubic $<$ octahedral $<$ spherical.

(A) (a)

(c)

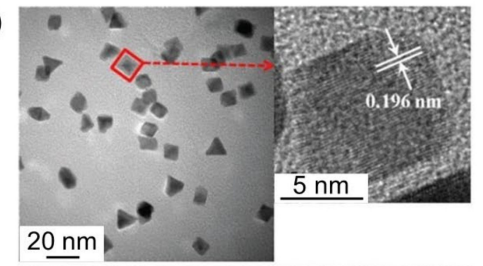

(b)

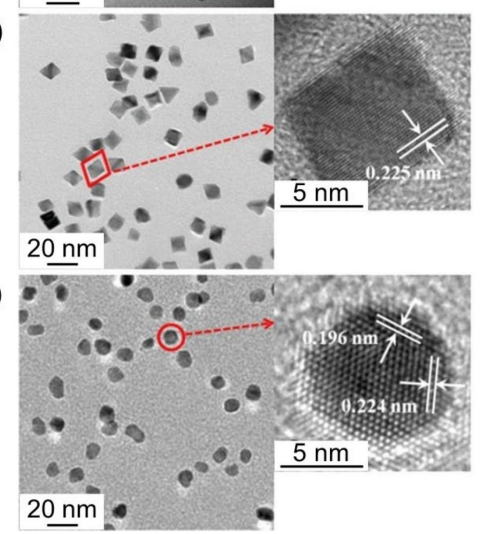

(B)

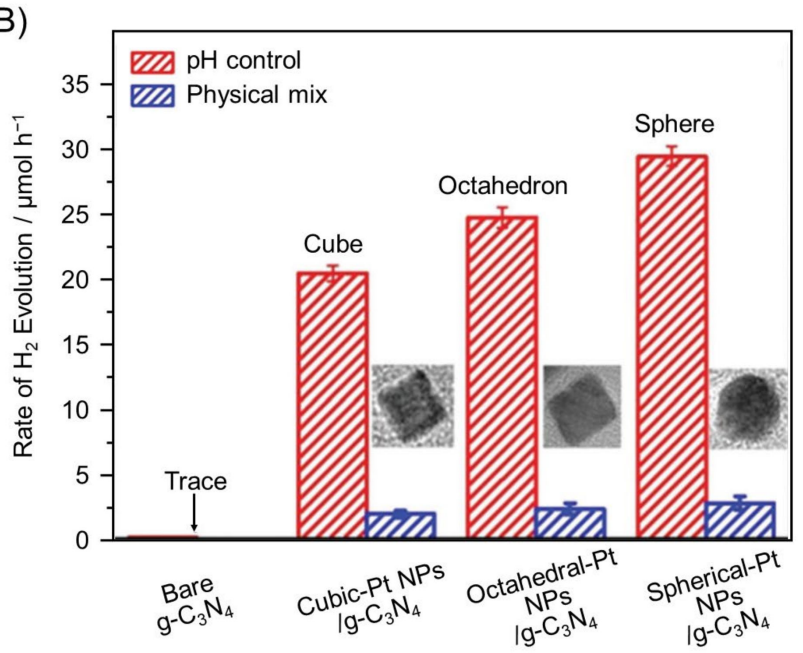

Figure 23. (A) TEM images and size distributions of dominant (a) cubic-, (b) octahedral-, and (c) spherical-Pt NPs. Insets show high-resolution (HR)-TEM images of a single Pt nanocrystal for each shape. (B) Photocatalytic $\mathrm{H}_{2}$ evolution activities of bare g- $\mathrm{C}_{3} \mathrm{~N}_{4}$, as-prepared Pt NPs/g- $\mathrm{C}_{3} \mathrm{~N}_{4}$ photocatalysts, and physically mixed samples under Vis-light irradiation $(\lambda>400 \mathrm{~nm})$ within $1 \mathrm{~h}$. Reproduced with permission from reference [221]. Copyright 2016 The Royal Society of Chemistry.

Similar shape dependence has also been observed for $\mathrm{TiO}_{2}$ loaded with Pd NPs. In 2018, $\mathrm{Yu}$ and co-workers prepared $\mathrm{Pd} \mathrm{NPs} / \mathrm{TiO}_{2}$ photocatalysts loaded with cubic or tetrahedral Pd nanocrystals (cubic-Pd NPs $/ \mathrm{TiO}_{2}$ and tetrahedral-Pd NPs $/ \mathrm{TiO}_{2}$, respectively; Figure 24A) and investigated their HER activity [222]. The results demonstrated that tetrahedral-Pd NPs $/ \mathrm{TiO}_{2}$ showed HER activity 1.5-2.0 times higher than that of cubic$\mathrm{Pd} \mathrm{NPs} / \mathrm{TiO}_{2}$ (Table 3). The tetrahedral-Pd NPs consisted of four (111) planes, to which photoexcited electron transfer from the $\mathrm{CB}$ of $\mathrm{TiO}_{2}$ proceeded efficiently (Figure 24B). On the tetrahedral-Pd NPs surface, adsorption of $\mathrm{H}, \mathrm{H}$-to- $\mathrm{H}_{2}$ conversion of molecules, and desorption of $\mathrm{H}_{2}$ molecules readily occurred (Figure 24C). Furthermore, theoretical calculations indicated that the (111) planes had a large work function, and that not only the edge/corner atoms but also the uncoordinated surface atoms, could be reaction sites. Accordingly, tetrahedral-Pd NPs $/ \mathrm{TiO}_{2}$ exhibited higher HER activity compared with cubic-Pd $\mathrm{NPs} / \mathrm{TiO}_{2}$ (Figure 24D). 
(A)

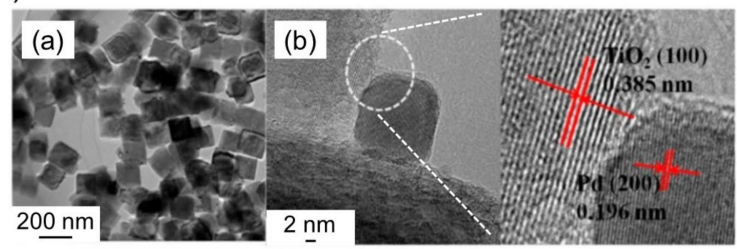

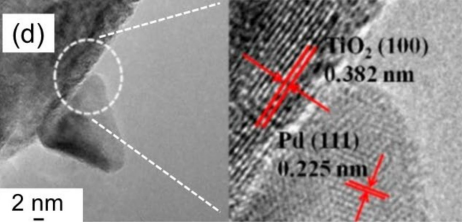

(B)

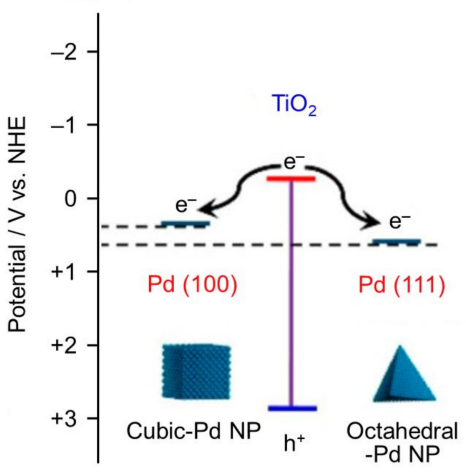

(C)

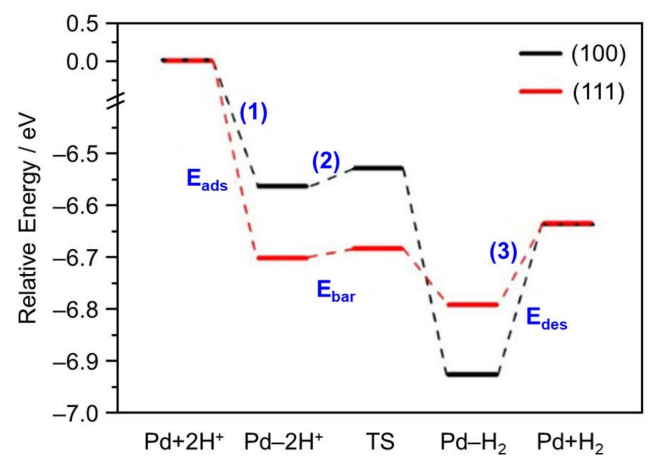

(D)

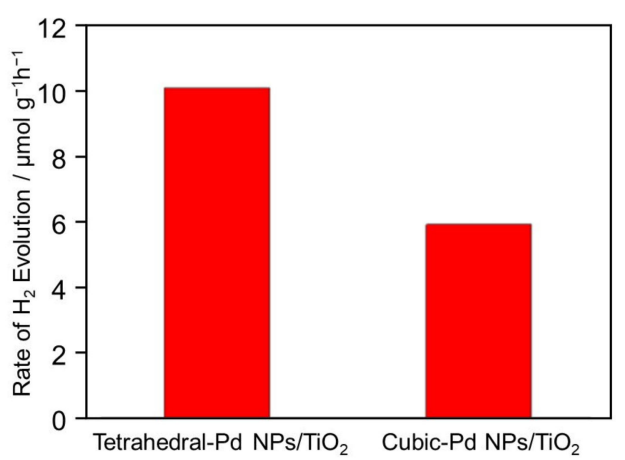

Figure 24. (A) TEM images of (a) cubic-Pd NPs/TiO and (c) tetrahedral-Pd NPs $/ \mathrm{TiO}_{2}$ samples, and HR-TEM images of (b) cubic-Pd NPs $/ \mathrm{TiO}_{2}$ and (d) tetrahedral-Pd NPs $/ \mathrm{TiO}_{2}$ samples. (B) Schematic illustration of photogenerated electron transfer from the $\mathrm{CB}$ of $\mathrm{TiO}_{2}$ to the $\mathrm{Pd}(100)$ and $\mathrm{Pd}(111)$ surface. (C) Energy variation in the $\mathrm{H}_{2}$ evolution process on $\mathrm{Pd}$ (100) and $\mathrm{Pd}$ (111) facets. The total energy of the initial Pd surface and two isolated $\mathrm{H}$ atoms is set to zero. Parenthesized numbers indicate the three reaction steps. TS represents the transition state. $E_{\mathrm{ads}}, E_{\mathrm{bar}}$, and $E_{\mathrm{des}}$ are the adsorption energy of $\mathrm{H}$ atoms, energy barrier, and desorption energy of the $\mathrm{H}_{2}$ molecule, respectively. The calculated $E_{\mathrm{abs}}, E_{\mathrm{bar}}$, and $E_{\mathrm{des}}$ of $\mathrm{Pd}(100)$ facets are $-6.57,0.034$, and $0.29 \mathrm{eV}$, respectively, and those of $\mathrm{Pd}$ (111) facets are $-6.77,0.018$ and $0.16 \mathrm{eV}$, respectively. (D) Photocatalytic $\mathrm{H}_{2}$ evolution activities of tetrahedral-Pd NPs $/ \mathrm{TiO}_{2}$ and cubic-Pd NPs $/ \mathrm{TiO}_{2}$ from $10 \mathrm{vol} \%$ lactic acid solution under Vis-light irradiation $(\lambda>420 \mathrm{~nm})$. Reproduced with permission from reference [222]. Copyright 2018 American Chemical Society.

In contrast, in a 2015 study on Pd NPs/cadmium sulfur (CdS), Yao and co-workers reported a different shape dependence than the above two reports [223]. In this study, the authors synthesized cubic-Pd NPs $(\sim 8.9 \mathrm{~nm})$ surrounded by six (100) planes and octahedral-Pd NPs $(\sim 6.0 \mathrm{~nm})$ surrounded by eight (111) planes (Figure 25A) and loaded them on CdS photocatalysts. From photocatalytic activity measurements, it was found that cubic-Pd NPs/CdS shows higher HER activity compared with octahedral-Pd NPs/CdS (Figure 25B). The photocurrent generation efficiency of cubic-Pd NPs/CdS was higher than that of octahedral-Pd NPs/CdS (Figure 25C), which indicated that electron transfer from the cocatalyst to the reactants was more efficient in the former. Estimation of the electrochemical surface area (ECSA) (Figure 25D) showed that the cubic-Pd NPs/CdS $\left(21.7 \mathrm{~m}^{2} \mathrm{~g}^{-1} \mathrm{Pd}\right)$ had an ECSA 1.49 times higher than that of octahedral-Pd NPs/CdS $\left(14.6 \mathrm{~m}^{2} \mathrm{~g}^{-1} \mathrm{Pd}\right)$, meaning that more proton adsorption/desorption sites were present in the former. These were attributed as the two main factors causing cubic-Pd NPs/CdS to show higher HER activity compared with octahedral-Pd NPs/CdS. 
(A)

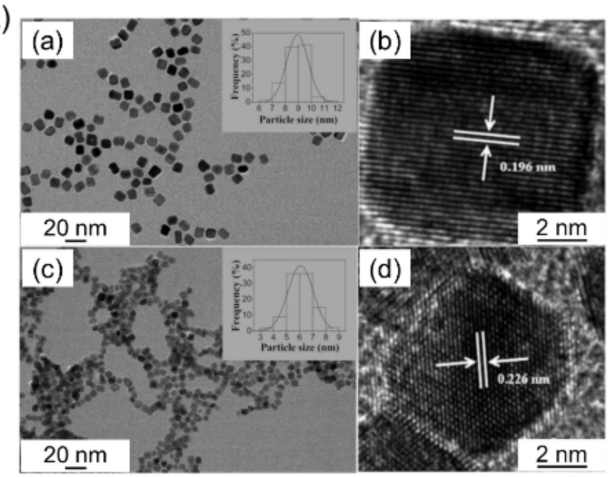

(C)

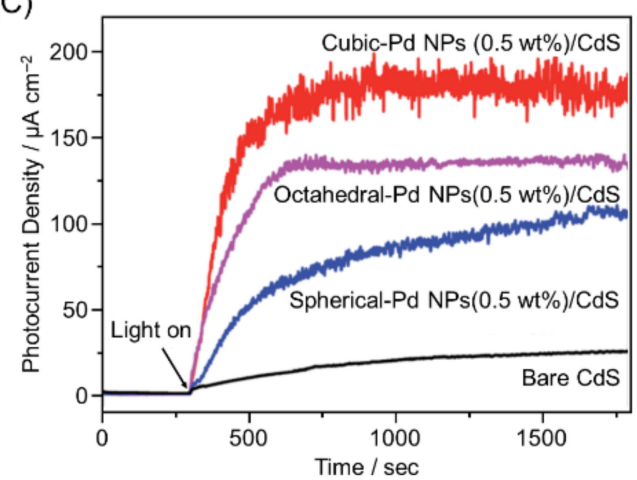

(B)

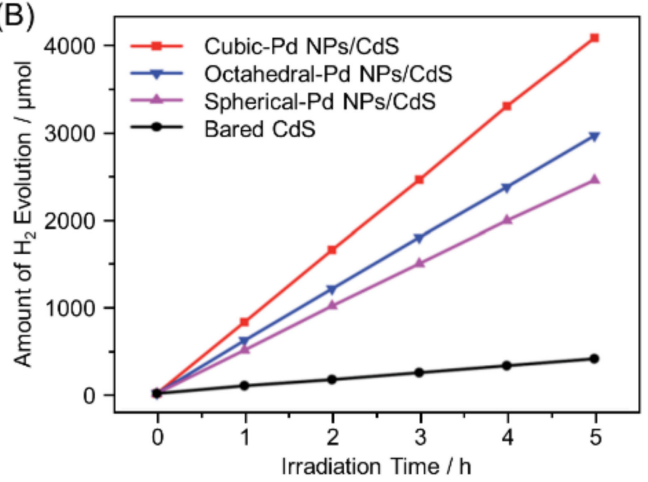

(D)

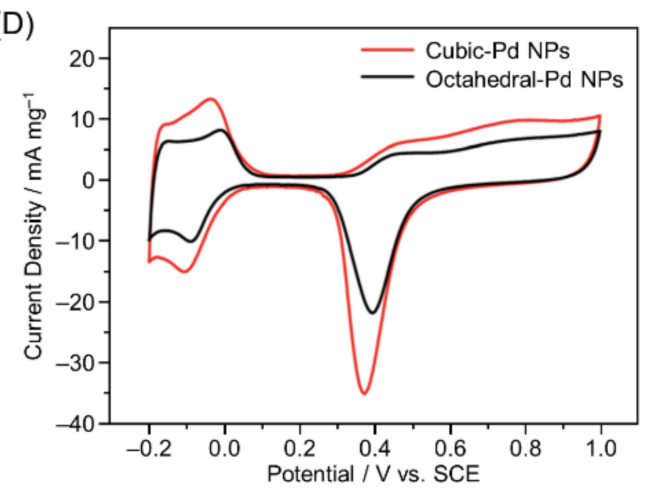

Figure 25. (A) TEM images of (a) cubic-Pd NPs and (c) octahedral-Pd NPs, and HR-TEM images of single (b) cubic-Pd NPs and (d) octahedral-Pd NPs. Insets in (a) and (c) are the size distributions of each Pd nanocrystal. (B) $\mathrm{H}_{2}$ evolution from $0.125 \mathrm{M}$ ammonium sulfite $\left(\left(\mathrm{NH}_{4}\right)_{2} \mathrm{SO}_{3}\right) \bullet \mathrm{H}_{2} \mathrm{O}$ aqueous solution under Vis-light irradiation $(\lambda>420 \mathrm{~nm})$ using cubic-Pd NPs/CdS, octahedral-Pd NPs/CdS, and spherical-Pd NPs/CdS photocatalysts. (C) Electron shuttle-mediated photocurrent measurements in suspensions of CdS and three types of Pd NPs-deposited CdS. Experimental conditions: $\mathrm{Pd} / \mathrm{CdS}$ concentration, $0.5 \mathrm{~g} \mathrm{~L}^{-1}$ of photolyte; aqueous photolyte composition, $0.1 \mathrm{M}$ of sodium nitrate $\left(\mathrm{NaNO}_{3}\right) / 1.0 \mathrm{mM}$ of $\mathrm{FeCl}_{3} / 10 \mathrm{vol} \% \mathrm{CH}_{3} \mathrm{OH}$; light source, $300 \mathrm{~W}$ Xe light with cutoff filter $(\lambda>420 \mathrm{~nm}$ ); bias potential, $0.6 \mathrm{~V}$ (vs. saturated calomel electrode (SCE)). (D) Cyclic voltammetry curves of cubic-Pd NPs and octahedral-Pd NPs derived from $0.1 \mathrm{M}$ of $\mathrm{HClO}_{4}$ solution at a scan rate of $50 \mathrm{mV} \mathrm{s}^{-1}$. Reproduced with permission from reference [223]. Copyright 2015 The Royal Society of Chemistry.

In the above studies, one metal element was used in the cocatalyst. However, in 2016, $\mathrm{Yao}, \mathrm{Xu}$, and co-workers reported using two elements in the cocatalyst. The authors found that the HER activity of Pt-Pd alloy NPs/CdS depended on both the shape and composition of the alloy NPs cocatalyst [224]. Regarding this shape dependence, cubic-PtPd NPs/CdS had a much higher HER activity (Figure 26A) and a 3.4 times higher photocatalytic turnover frequency (TOF) compared with octahedral-PtPd NPs/CdS. Electrochemical experiments (Figure 26B) showed that the interfacial electron transfer rate in cubic-PtPd NPs/CdS was higher than that in octahedral-PtPd NPs/CdS. Regarding the composition dependence, $\mathrm{H}_{2}$ evolution and the TOF were enhanced when the atomic ratio of $\mathrm{Pt}$ to $\mathrm{Pd}$ was changed from 1:0 to about 2:1. 
(A)

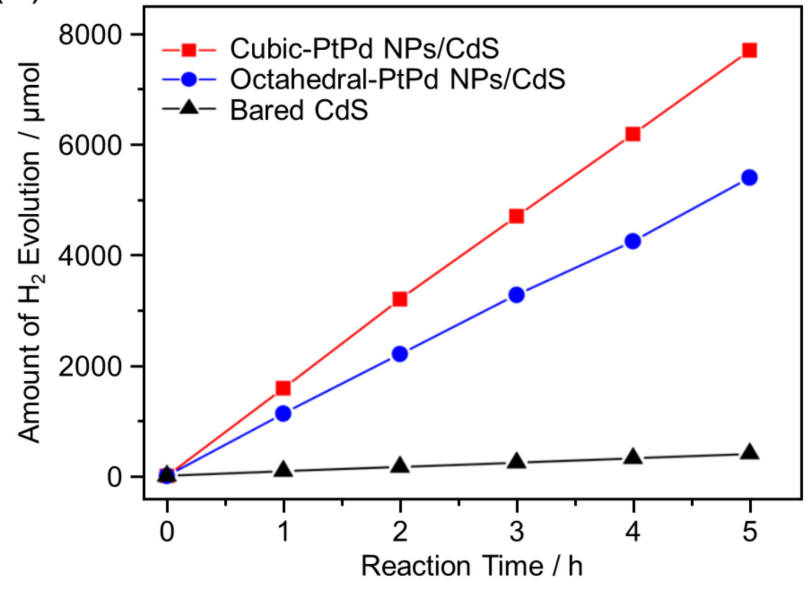

(B)

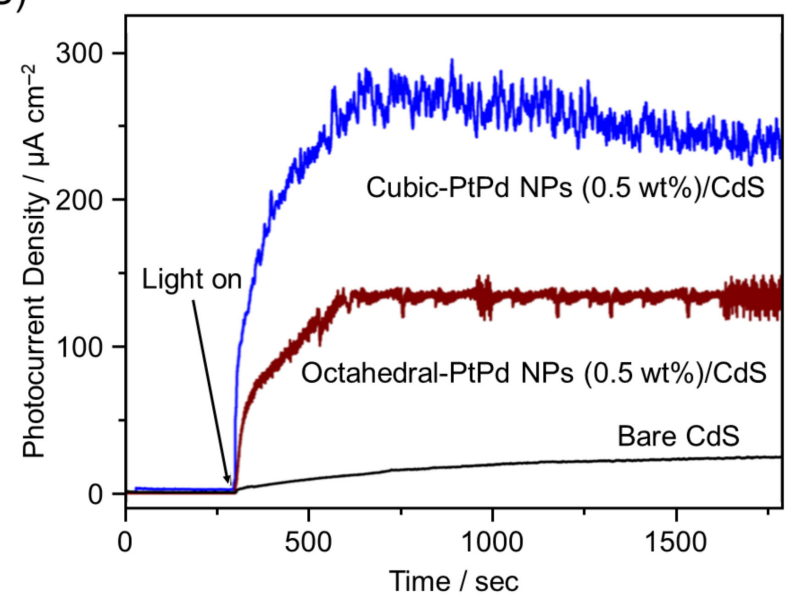

Figure 26. (A) Irradiation time course for $\mathrm{H}_{2}$ evolution from $1.0 \mathrm{M}$ of aqueous $\left(\mathrm{NH}_{4}\right)_{2} \mathrm{SO}_{3}$ solution under Vis-light irradiation $(\lambda>420 \mathrm{~nm})$ using cubic-PtPd NPs/CdS, octahedral-PtPd NPs/CdS, and bare-CdS photocatalysts. (B) Electron shuttle-mediated photocurrent measurements in suspensions of CdS, cubic-PtPd NPs/CdS, and octahedral-PtPd NPs/CdS. Conditions: Catalyst concentration, $0.5 \mathrm{~g} \mathrm{~L}^{-1}$ of photolyte; aqueous photolyte composition, $0.1 \mathrm{M}$ of $\mathrm{NaNO}_{3} / 1.0 \mathrm{mM}$ of $\mathrm{FeCl}_{3} / 10 \mathrm{vol} \%$ $\mathrm{CH}_{3} \mathrm{OH}$; light source, $300 \mathrm{~W}$ Xe light with a cutoff filter $(\lambda>420 \mathrm{~nm}$ ); bias potential, $0.6 \mathrm{~V}$ (vs. SCE)). Reproduced with permission from reference [224]. Copyright 2016 American Chemical Society.

\subsection{Interfacial Structure Control}

Improving bonding between the cocatalyst and photocatalyst surface is also effective in enhancing the photocatalytic activity. In 2021, Domen, Teshima, and co-workers established a method for loading highly dispersed and uniformly sized HER cocatalysts. In this method, Pt NPs were first loaded on $\mathrm{BaTaO}_{2} \mathrm{~N}$ (Figure 7 and Table 3) by impregnation, followed by additional loading of Pt on Pt NPs by photodeposition (Figure 27A) [225]. This sequential loading strongly immobilized Pt NPs on $\mathrm{BaTaO}_{2} \mathrm{~N}$, which facilitated the transfer of photoexcited electrons from the semiconductor to the cocatalyst, and the resulting photocatalyst showed high HER activity (AQY $=6.8 \pm 0.5 \%$ at $420 \mathrm{~nm}$; Figure $27 \mathrm{~B}$ and Table 3). The Z-scheme water-splitting reaction using $\mathrm{Pt} \mathrm{NPs} / \mathrm{BaTaO}_{2} \mathrm{~N}$ as the HER photocatalyst and tungsten oxide $\left(\mathrm{WO}_{3}\right)$ as the OER photocatalyst (Figure $4 \mathrm{~B}$ ) showed an AQY $=4.0 \%$ (at $420 \mathrm{~nm}$ ) and $\mathrm{STH}=0.24 \%$.

\subsection{Surface Structure Control}

The HER proceeds on the noble metal (such as Pt and Rh) NPs cocatalyst. However, when $\mathrm{O}_{2}$ is present in the system, the reverse reaction also proceeds in parallel $\left(\mathrm{O}_{2}\right.$ photoreduction and reverse reaction; see Figure 28) on the noble metal NPs cocatalyst. Therefore, to efficiently produce $\mathrm{H}_{2}$, the reverse reaction on the noble metal NPs cocatalyst must be suppressed. For this purpose, effective methods are as follows: (i) formation of a reverse-reaction-suppressing layer on the cocatalyst, (ii) formation of a reverse-reactionsuppressing layer on the entire photocatalyst surface, and (iii) giving the cocatalyst itself a reverse-reaction-suppressing function. Such methods are described in the following section. 
(A)

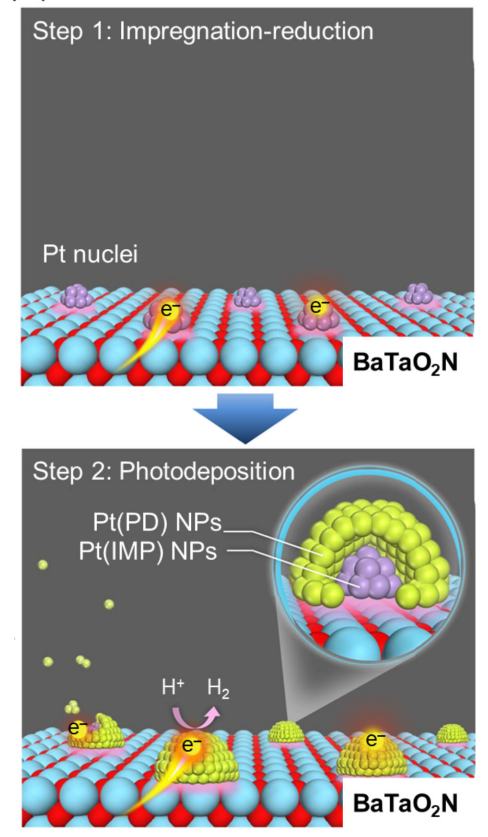

(B)

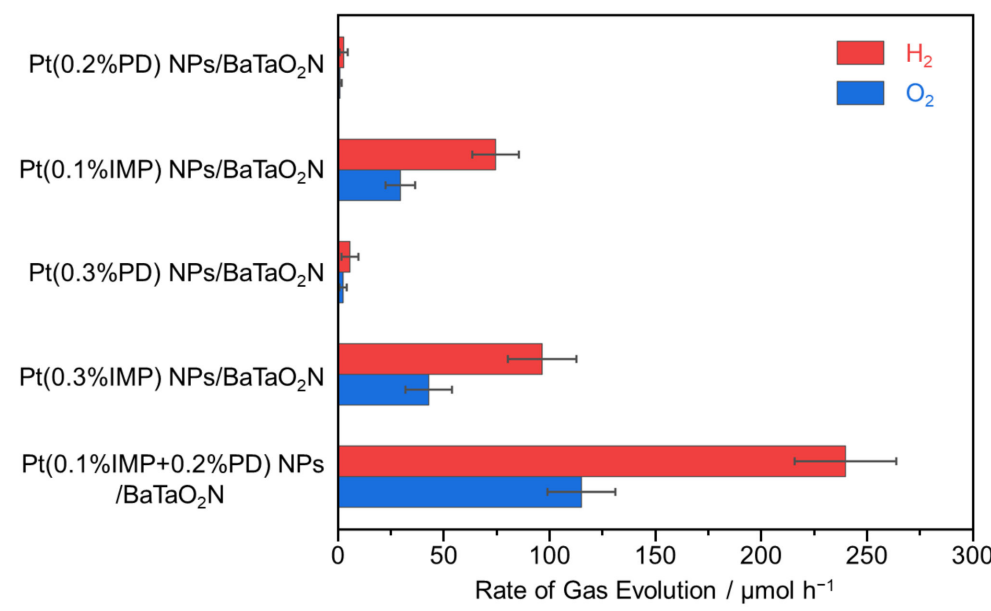

Figure 27. (A) Interaction between Pt NPs and $\mathrm{BaTaO}_{2} \mathrm{~N}$ photocatalyst. Schematic of sequential Pt NPs cocatalyst deposition on $\mathrm{BaTaO}_{2} \mathrm{~N}$. (B) $\mathrm{H}_{2}$ and $\mathrm{O}_{2}$ evolution rates during Z-scheme watersplitting reaction using $\mathrm{Pt} \mathrm{NPs} / \mathrm{BaTaO}_{2} \mathrm{~N}$ as the HER photocatalyst. For example, Pt $(0.2 \% \mathrm{PD}) \mathrm{NPs}$ and $\mathrm{Pt}(0.1 \% \mathrm{IMP}) \mathrm{NPs}$ represent $\mathrm{Pt}$ NPs loaded on $\mathrm{BaTaO}_{2} \mathrm{~N}$ by photodeposition and impregnation methods, respectively. Error bars indicate standard deviation of three measurements. Conditions: $\mathrm{Pt} \mathrm{NP} / \mathrm{BaTaO}_{2} \mathrm{~N}$ photocatalyst, $0.1 \mathrm{~g}$; surface-treated $\mathrm{WO}_{3}, 0.15 \mathrm{~g} ; 1 \mathrm{mM}$ of aqueous NaI solution, $150 \mathrm{~mL}$; light source, $300 \mathrm{~W}$ Xe lamp $(\lambda \geq 420 \mathrm{~nm}$ ) or solar simulator (AM1.5G); irradiation area for solar simulator, $7.6 \mathrm{~cm}^{2}$; reaction system, Pyrex top-illuminated vessel connected to the closed gas-circulation system with the periodical evacuation of gas products. Reproduced with permission from reference [225]. Copyright 2021 Springer Nature Limited.

(C) $\mathrm{O}_{2}$ photoreduction reaction

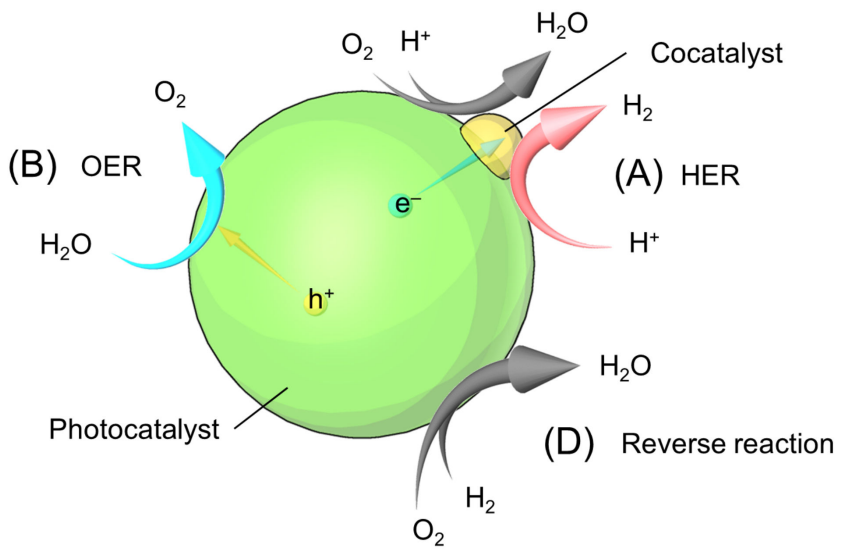

Figure 28. Possible reactions that occur over a water-splitting photocatalyst during the photocatalytic reaction: (A) HER, (B) OER, (C) $\mathrm{O}_{2}$ photoreduction reaction, and (D) reverse reaction [79].

\subsubsection{Formation of $\mathrm{Cr}_{2} \mathrm{O}_{3}$ Shell on Cocatalysts}

In 2006, Maeda, Domen, and co-workers found that formation of a $\mathrm{Cr}_{2} \mathrm{O}_{3}$ layer on the Rh NPs' surface (Figure 29) suppressed one of the reverse reactions, namely, the $\mathrm{O}_{2}$ photoreduction reaction (Figure 28C) [226-230]. Various experiments on the reaction mechanism showed that $\mathrm{H}_{2} \mathrm{O}$ and $\mathrm{H}^{+}$ions, which are polar molecules, penetrated the $\mathrm{Cr}_{2} \mathrm{O}_{3}$ layer because this layer was hydrated in water, resulting in $\mathrm{H}_{2}$ being generated on 
the Rh surface. On the other hand, $\mathrm{O}_{2}$, which is a nonpolar molecule, cannot penetrate the $\mathrm{Cr}_{2} \mathrm{O}_{3}$ layer from the outside, meaning that the formation of this layer prevented the reverse reaction. This inhibition of the reverse reaction has also been observed when other noble NPs, such as $\mathrm{Cu}, \mathrm{Pd}, \mathrm{Pt}$, and $\mathrm{Au}$, were used as cocatalysts $[75,232,233]$.

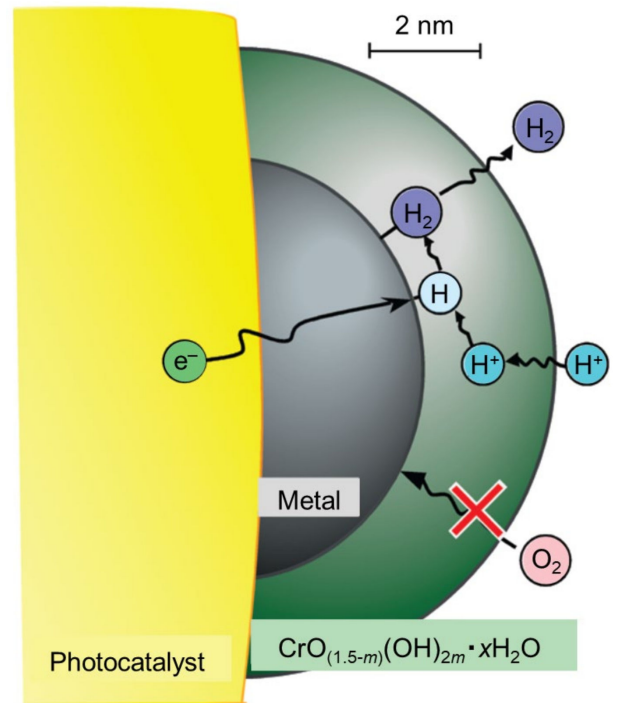

Figure 29. Schematic model of HER on core-shell $\mathrm{Cr}_{2} \mathrm{O}_{3}$ /noble-metal NPs system as a cocatalyst for photocatalytic overall water splitting. Reproduced with permission from reference [232]. Copyright 2009 American Chemical Society.

3.5.2. Formation of Other Amorphous Metal (Oxy) Hydroxide Layers on the Photocatalyst Surface

The reverse reactions can also be suppressed by loading amorphous oxyhydroxides, such as $\mathrm{TiO}_{2}, \mathrm{Nb}_{2} \mathrm{O}_{5}$, and $\mathrm{Ta}_{2} \mathrm{O}_{5}$, on the entire surface of the photocatalyst particles [231]. These layers were created by loading peroxide complexes onto the photocatalytic surface by photodeposition. This reaction is a downhill reaction that proceeds more easily than the formation of $\mathrm{Cr}_{2} \mathrm{O}_{3}$ layers described above using an uphill reaction (in this case, photodeposition using $\mathrm{CrO}_{4}{ }^{2-}$ ). Therefore, this method can be applied to water-splitting photocatalysts that have weak reducing and oxidizing power owing to their narrow BG.

For example, in 2015, Takata, Domen, and co-workers mixed $\mathrm{TiO}_{2}$ and $\mathrm{RhCrO}_{x}$ $\mathrm{NPs} / \mathrm{LaMg}_{1 / 3} \mathrm{Ta}_{2 / 3} \mathrm{O}_{2} \mathrm{~N}(\mathrm{Mg}=$ magnesium $)$ in hydrogen peroxide $\left(\mathrm{H}_{2} \mathrm{O}_{2}\right)$ solution and irradiated them with light to form a core-shell structure in which $\mathrm{TiOXH}(\mathrm{OXH}=$ oxyhydroxide) covered the entire surface of the semiconductor photocatalyst $\left(\mathrm{LaMg}_{1 / 3} \mathrm{Ta}_{2 / 3} \mathrm{O}_{2} \mathrm{~N}\right)$ and the cocatalyst particles $\left(\mathrm{RhCrO}_{x} \mathrm{NPs}\right)$ (Figure 30A). The authors found that this suppressed the $\mathrm{O}_{2}$ reduction reaction in the as-obtained $\mathrm{TiOXH} / \mathrm{RhCrO}_{x} \mathrm{NPs} / \mathrm{LaMg}_{1 / 3} \mathrm{Ta}_{2 / 3} \mathrm{O}_{2} \mathrm{~N}$ [234] The authors also succeeded in forming a double-coating layer consisting of $\mathrm{SiOXH}$ and $\mathrm{TiOXH}$ on the surface of $\mathrm{LaMg}_{1 / 3} \mathrm{Ta}_{2 / 3} \mathrm{O}_{2} \mathrm{~N}$ using a similar preparation method (Figure 30A). In this case, the amount of $\mathrm{H}_{2}$ and $\mathrm{O}_{2}$ evolution increased linearly with irradiation time (Figure 30B). Double coating resulted in the formation of a more uniform layer. Additionally, when $\mathrm{SiOXH}$ was mixed into the layer, the hydrophilicity of the layer increased, further inhibiting the permeation of $\mathrm{O}_{2}$ molecules. These factors resulted in more effective suppression of the reverse reaction. 
(A)

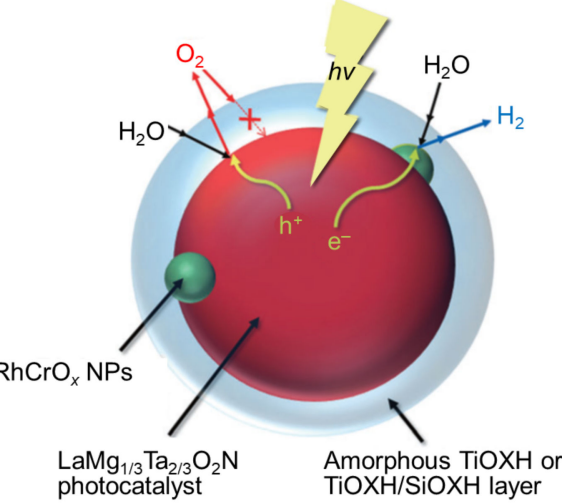

(C)

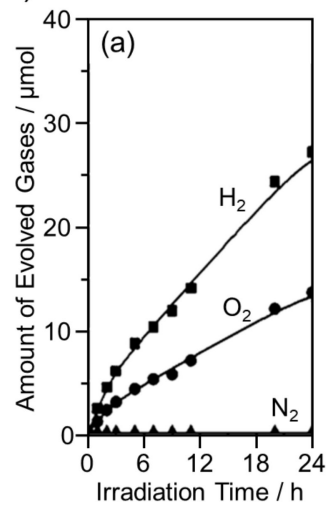

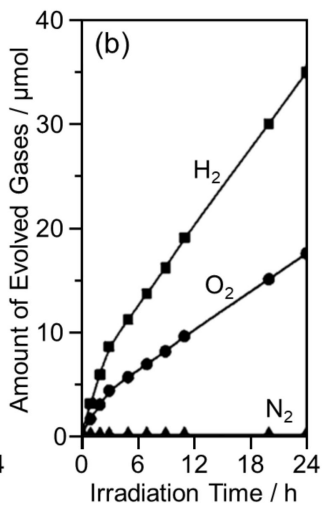

(B)

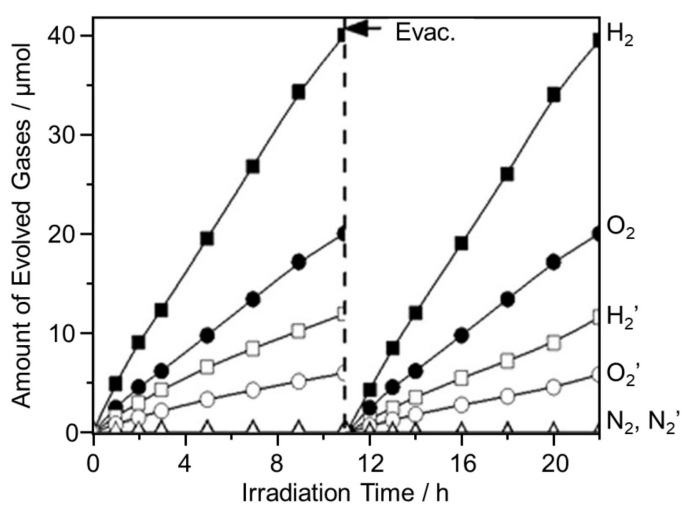

(D)

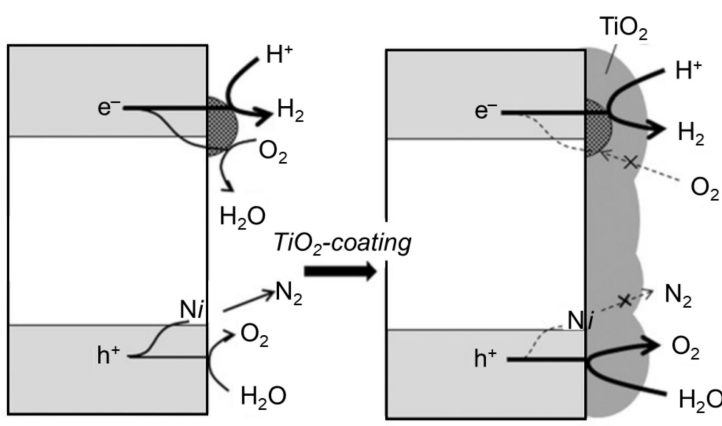

Figure 30. (A) Reaction mechanism for water splitting on a surface-coated photocatalyst. (B) Gas evolution during water splitting on $\mathrm{TiOXH} / \mathrm{SiOXH} / \mathrm{RhCrO} \mathrm{NPs}_{x} / \mathrm{LaMg}_{1 / 3} \mathrm{Ta}_{2 / 3} \mathrm{O}_{2} \mathrm{~N}$ under UV/Vislight irradiation ( $\lambda>300 \mathrm{~nm}$; filled symbols) and Vis-light irradiation alone $(\lambda>420 \mathrm{~nm}$; open symbols). Reaction conditions: Catalyst $(0.2 \mathrm{~g})$, reaction solvent (pure water, $250 \mathrm{~mL})$, Xe lamp (300 W); a side-irradiation-type Pyrex reaction vessel was used. (C) Time courses of gas evolution on $\mathrm{RhCrO}_{x} \mathrm{NPs} / \mathrm{LaMg}_{1 / 3} \mathrm{Ta}_{2 / 3} \mathrm{O}_{2} \mathrm{~N}$ modified by different $\mathrm{TiO}_{2}$ coating methods: (a) $\mathrm{TiO}_{2} / \mathrm{H}_{2} \mathrm{O}_{2}$ and (b) TTIP $/ \mathrm{H}_{2} \mathrm{O}_{2}$. Reaction conditions are the same as (A). (D) Schematic of the functions of the cocatalyst $\left(\mathrm{RhCrO}_{x}\right)$ and the coating layer $\left(\mathrm{TiO}_{2}\right)$. N, unstable surface nitrogen; $\mathrm{N}_{i}$, nitrogen at the interface. Reproduced with permission from references [234,235]. Copyright $2015 \mathrm{Wiley}-\mathrm{VCH}$ and 2016 Wiley-VCH.

In 2016, these authors also reported the effect of the type of precursor on the function of the photocatalyst. Changing the precursor from $\mathrm{TiO}_{2}$ to titanium tetraisopropoxide (TTIP) was found to enhance the water-splitting activity of the resulting photocatalyst 1.4 times (Figure 30C) [235]. This paper also reported that the formation of TiOXH layers enhanced the stability of $\mathrm{LaMg}_{1 / 3} \mathrm{Ta}_{2 / 3} \mathrm{O}_{2} \mathrm{~N}$ during photocatalytic reactions (Figure 30D).

\subsubsection{Formation of Solid Solution ( $\mathrm{Rh}-\mathrm{Cr}, \mathrm{Rh}-\mathrm{Zr})$ Oxide Cocatalysts}

In 2006, Maeda and Domen showed that suppression of the reverse reaction due to $\mathrm{Cr}_{2} \mathrm{O}_{3}$ also occurred when the solid-solution structure was formed instead of the core-shell structure [236-240]. Such a suppression effect of $\mathrm{Rh}_{2-x} \mathrm{Cr}_{x} \mathrm{O}_{3} \mathrm{NPs}$ on the reverse reaction was also observed in our study on UV-light-driven photocatalysis (Figure 19) [77].

Furthermore, long-term light irradiation of the obtained photocatalyst led to the dissolution of $\mathrm{Cr}^{6+}$ ions [284]. This results in decreased catalytic activity and is considered to have an impact on environmental destruction and health hazards. Therefore, Saruyama, Teranishi, and co-workers worked to create cocatalysts with elements other than $\mathrm{Cr}$ that have similar effects. As a result, in 2020, these authors found that the $\mathrm{RhZrO}_{x}$ solid-solution NPs cocatalyst, which contained $\mathrm{Zr}$ and $\mathrm{Rh}$, also had the ability to suppress the reverse reaction [241]. Although UV-light-driven aluminum (Al)-doped $\mathrm{SrTiO}_{3}\left(\mathrm{SrTiO}_{3}: \mathrm{Al}\right)$ [285] 
was used as a photocatalyst in this study, future studies are expected to be conducted on Vis-light-driven water-splitting photocatalysts using the same NPs as a cocatalyst.

\subsection{Charge-State Control}

The function of the cocatalyst also depends on the charge state of the metal contained in the cocatalyst. Cobalt oxide $\left(\mathrm{CoO}_{x}\right)$ is often used as an OER cocatalyst owing to its high efficiency, low cost, and earth abundance. Yamakata, Maeda, and co-workers reported in 2020 that g- $\mathrm{C}_{3} \mathrm{~N}_{4}$ loaded with $\mathrm{Co}^{2+}$-based spinel-type $\mathrm{CoAl}_{2} \mathrm{O}_{4}$ NPs cocatalysts (5-20 nm) showed higher OER activity compared with g- $\mathrm{C}_{3} \mathrm{~N}_{4}$ loaded with spinel-type $\mathrm{Co}_{3} \mathrm{O}_{4} \mathrm{NPs}$ cocatalysts $(5-20 \mathrm{~nm})$ consisting of a mixture of $\mathrm{Co}^{2+}$ and $\mathrm{Co}^{3+}$ (Figure 31A) [242]. The results of transient absorption spectroscopy showed that the stronger hole-trapping effect in $\mathrm{CoAl}_{2} \mathrm{O}_{4} \mathrm{NPs}$ compared with $\mathrm{Co}_{3} \mathrm{O}_{4}$ cocatalysts caused this phenomenon (Figure 31B).

(A) (a) $\mathrm{CoAl}_{2} \mathrm{O}_{4} \mathrm{NPs} / \mathrm{g}-\mathrm{C}_{3} \mathrm{~N}_{4}$

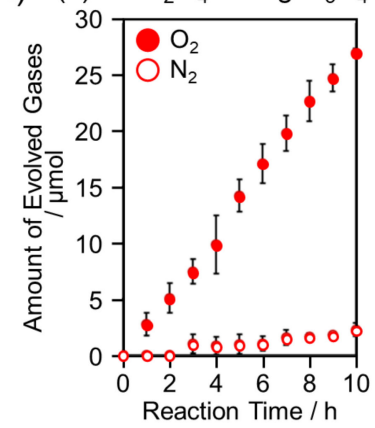

(b) $\mathrm{Co}_{3} \mathrm{O}_{4} \mathrm{NPs} / \mathrm{g}-\mathrm{C}_{3} \mathrm{~N}_{4}$

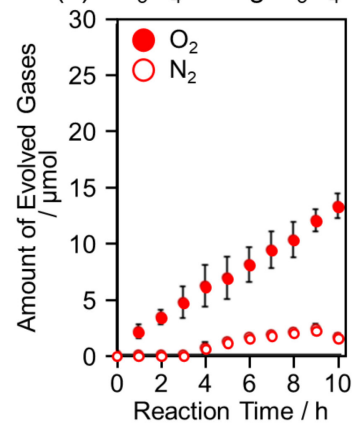

(B)

(a) $4000 \mathrm{~cm}^{-1}$

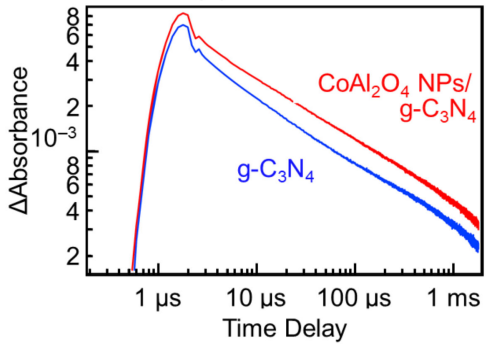

(b) $6000 \mathrm{~cm}^{-1}$

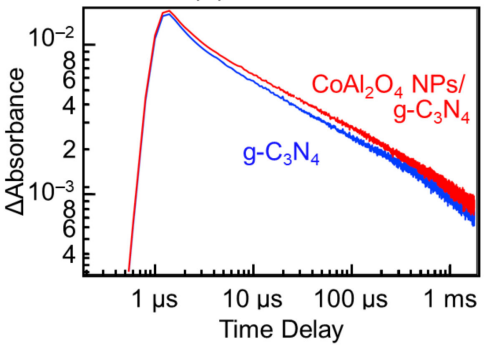

(c) $9000 \mathrm{~cm}^{-1}$

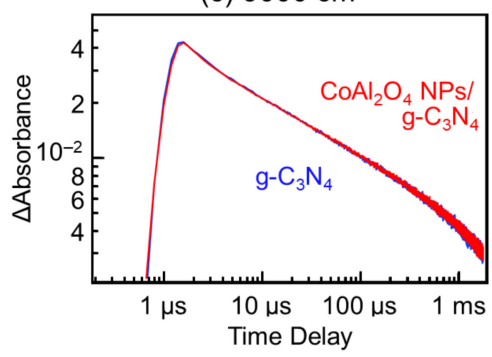

Figure 31. (A) Time course of photocatalytic $\mathrm{O}_{2}$ evolution over g- $\mathrm{C}_{3} \mathrm{~N}_{4}$ loaded with (a) $\mathrm{CoAl}_{2} \mathrm{O}_{4}$ (3.0 wt\%) NPs and (b) $\mathrm{Co}_{3} \mathrm{O}_{4}(1.5 \mathrm{wt} \%) \mathrm{NPs}$ cocatalysts. Reaction conditions: catalyst, $50 \mathrm{mg} ; \mathrm{La}_{2} \mathrm{O}_{3}$, $200 \mathrm{mg}$; reactant solution, aqueous $\mathrm{AgNO}_{3}(10 \mathrm{mM}, 140 \mathrm{~mL})$; light source, $300 \mathrm{~W}$ Xe lamp with cut-off filter (L42). (B) Decay curves for transient absorption intensity at different wavenumbers for $\mathrm{g}-\mathrm{C}_{3} \mathrm{~N}_{4}$ and $\mathrm{CoAl}_{2} \mathrm{O}_{4}(3.0 \mathrm{wt} \%) \mathrm{NPs} / \mathrm{g}-\mathrm{C}_{3} \mathrm{~N}_{4}$. The decay kinetics of the more reactive electrons recorded at $4000 \mathrm{~cm}^{-1}$ (a) became slower after modification of $\mathrm{CoAl}_{2} \mathrm{O}_{4} \mathrm{NPs}$ on the surface of $\mathrm{g}-\mathrm{C}_{3} \mathrm{~N}_{4}$, indicating that the population of surviving electrons was increased by loading with $\mathrm{CoAl}_{2} \mathrm{O}_{4} \mathrm{NPs}$. However, such a slow decay was not clear at $6000 \mathrm{~cm}^{-1}(\mathbf{b})$, and not observed at $9000 \mathrm{~cm}^{-1}(\mathbf{c})$. These results strongly suggest that photogenerated holes in g- $\mathrm{C}_{3} \mathrm{~N}_{4}$ could move to the loaded $\mathrm{CoAl}_{2} \mathrm{O}_{4}$ $\mathrm{NPs}$, thereby increasing the electron population. Reproduced with permission from reference [242]. Copyright 2020 American Chemical Society.

Similar results have been reported by Liu, Yang, and co-workers. In 2021, these authors successfully loaded $\mathrm{Co}^{2+}$-based $\mathrm{CoO}_{x}$ cocatalysts $(\sim 2.5 \mathrm{~nm})$ or $\mathrm{Co}^{3+}$-based $\mathrm{CoO}_{x}$ cocatalysts $(\sim 2.6 \mathrm{~nm})$ on TaON photocatalysts using a photochemical metal-organic deposition (PMOD) method [243]. Photocatalytic studies showed that $\mathrm{Co}^{2+}$-based $\mathrm{CoO}_{x}$ cocatalysts were 1.6 times more effective for OER than $\mathrm{Co}^{3+}$-based $\mathrm{CoO}_{x}$ cocatalysts and that TaON loaded with $\mathrm{Co}^{2+}$-based $\mathrm{CoO}_{x}$ cocatalysts had an AQY of $21.2 \%$ (at $420 \pm 15 \mathrm{~nm}$; Figure 32A and Table 3). Photoelectrochemical reactions and photoluminescence (PL) measurements indicated that $\mathrm{Co}^{2+}$ species played an important role in accelerating charge separation and transport (Figure 32B). 
(A)

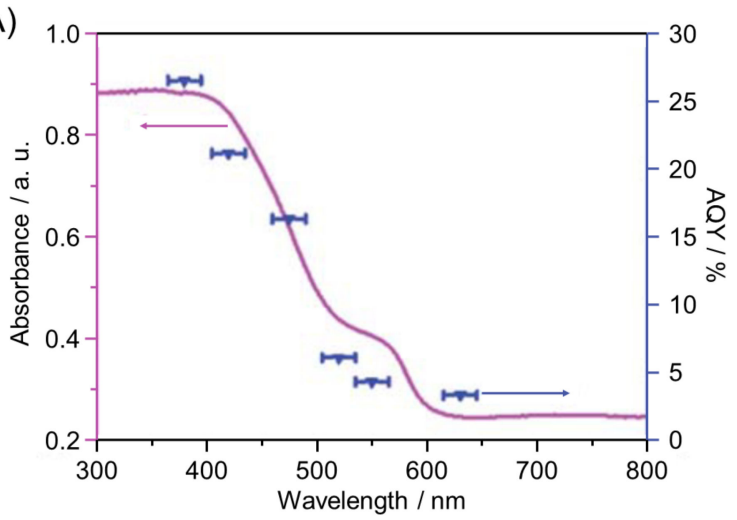

(B)

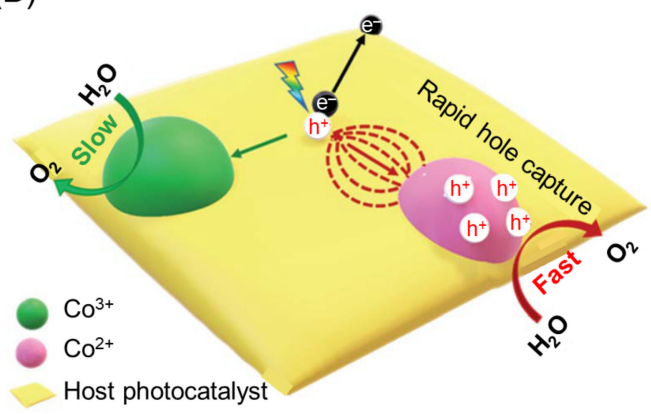

Figure 32. (A) Absorption spectrum and wavelength-dependent AQY of photocatalytic $\mathrm{O}_{2}$ evolution for $\mathrm{CoO}_{x}\left(\mathrm{Co}^{2+}\right) \mathrm{NPs} / \mathrm{TaON}$. (B) Proposed mechanism of the photocatalytic water-oxidation process of $\mathrm{CoO}_{x} \mathrm{NPs} / \mathrm{TaON}$, indicating accelerated hole transportation and reaction on the $\mathrm{Co}^{2+}$ species. Reproduced with permission from reference [243]. Copyright 2021 The Royal Society of Chemistry.

\section{Separation of Each Reaction Site}

To create highly active water-splitting photocatalysts, exciton recombination must be suppressed [286]. Therefore, in recent years, the development of water-splitting photocatalysts in which the excited electrons and holes are spatially separated has been promoted, such that the reduction and oxidation reactions can proceed on different surfaces. One approach is to load both the HER and OER cocatalysts on the photocatalyst (dualcocatalyst loading). With such a dual-cocatalyst loading, the excited electrons and holes transfer toward the respective cocatalysts. As a result, they can be spatially separated, and the water-oxidation and water-reduction reactions can proceed on different surfaces of the photocatalyst. However, when loading of the dual-cocatalyst is conducted randomly, the two types of cocatalysts cannot be spatially separated, thereby causing the recombination of excitons and reverse reactions to proceed, which might decrease the photocatalytic activity (Figure 33A). Therefore, loading cocatalysts onto suitable sites for each reaction is essential. The following methods can somewhat avoid the exciton recombination and reverse reactions proceeding: (i) forming facets that both excited electrons and holes can easily reach (Section 4.1; Figure 33B) $[287,288]$; (ii) forming onedimensional structures (Section 4.2; Figure 33C) [289,290]; (iii) forming a yolk-shell structure (Section 4.3; Figure 33D) [291-293]; and (iv) forming a built-in electric field in the band structure (Section 4.4; Figure 33E) [141,155]. For some of the semiconductor photocatalysts described in this section, the appropriate cocatalysts, available reactions (OWSR, HER, or OER; Figure 4), activities, and references are summarized in Table 4 [287-293]. 

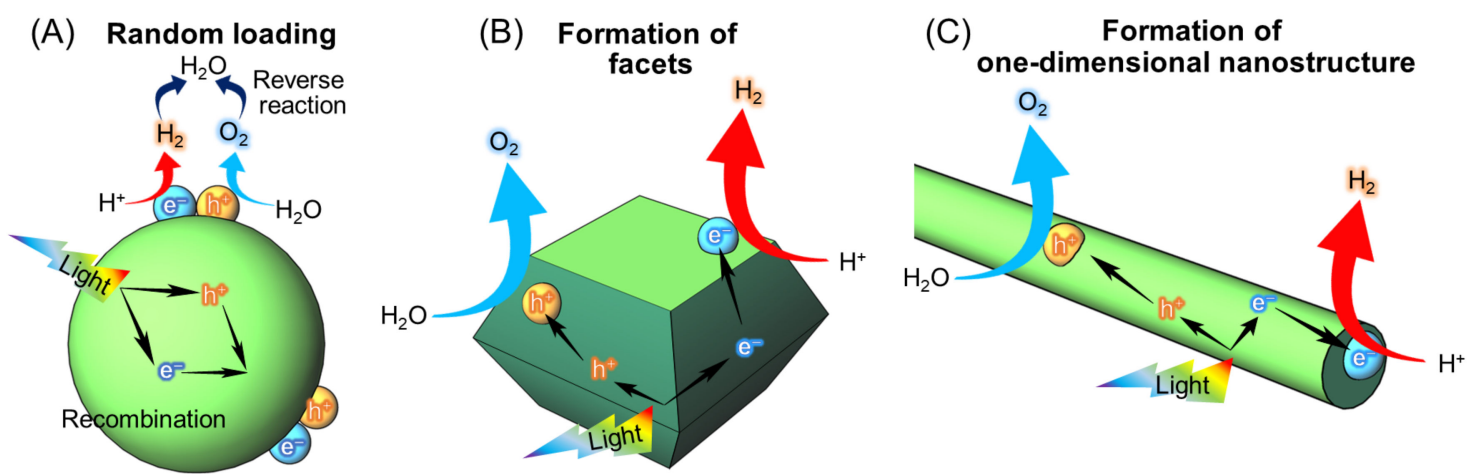

(D)

Formation of

yolk-shell (hollow) nanostructure
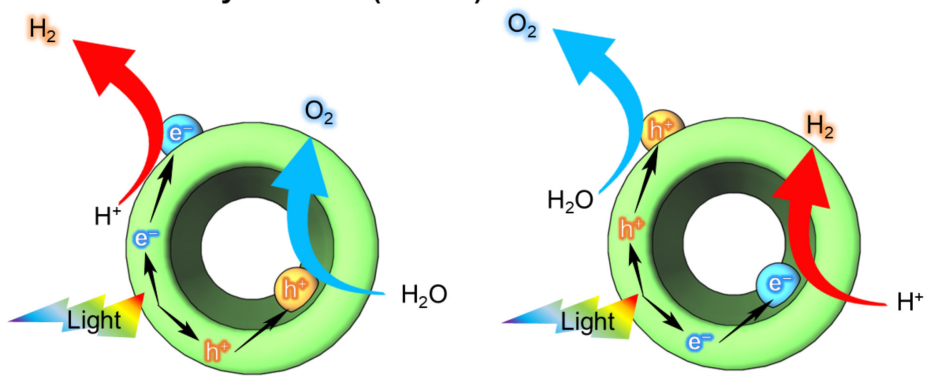

(E)

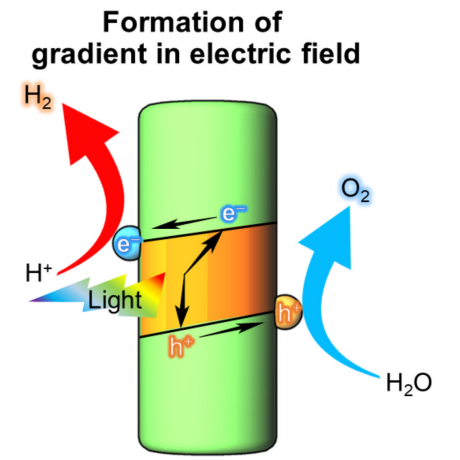

Figure 33. Schematic illustrations of the functionalization of photocatalysts by separating each reaction site. (A) Random loading of cocatalysts, (B) formation of facets that both excited electrons and holes can easily reach (Section 4.1), (C) formation of one-dimensional nanostructure (Section 4.2), (D) formation of yolk-shell (hollow) nanostructure (Section 4.3), and (E) formation of gradient in electric field (Section 4.4).

Table 4. Effective cocatalysts, possible reaction, and efficiency of water-splitting photocatalysts (Vis-light-driven) introduced in Section 4.

\begin{tabular}{|c|c|c|c|c|c|}
\hline Photocatalyst & $\begin{array}{l}\text { Cocatalyst } \\
(/ \mathbf{w t} \%)^{a}\end{array}$ & $\begin{array}{l}\text { Size } \\
/ \mathrm{nm}\end{array}$ & $\begin{array}{l}\text { Possible } \\
\text { Reaction } b\end{array}$ & Efficiency $^{c}$ & Ref. \\
\hline $\mathrm{BiVO}_{4}$ & $\begin{array}{c}\mathrm{Pt}(\mathrm{PD}) \mathrm{NPs} \\
-\mathrm{MnO}_{x}(\mathrm{PD}) \mathrm{NPs} \\
(\mathrm{Pt}: 0.1, \mathrm{Mn}: 0.1)\end{array}$ & $\begin{array}{c}\text { Pt: } 10-30 \\
\mathrm{MnO}_{x}: \mathrm{N} / \mathrm{A}\end{array}$ & $\begin{array}{c}\text { OER } \\
(\mathrm{pH}=6.8)\end{array}$ & $\begin{array}{c}\mathrm{ER}=\sim 660 \mu \mathrm{mol} \mathrm{h}^{-1} \mathrm{~g}^{-1} \\
(>420 \mathrm{~nm})\end{array}$ & [287] \\
\hline CdSe NRs & $\begin{array}{l}\text { Pt NPs-PdS NPs } \\
\text { (Pt: 0.5, PdS: 0.5) }\end{array}$ & $\begin{array}{l}\text { Pt: } \sim 2-6 \\
\text { PdS: N/A }\end{array}$ & HER & $\begin{array}{l}\mathrm{AQY}=\sim 45.0 \% \\
\quad(\text { at } 420 \mathrm{~nm})\end{array}$ & [289] \\
\hline $\mathrm{Cd}_{0.5} \mathrm{Zn}_{0.5} \mathrm{~S}: \mathrm{P}$ NRs & $\begin{array}{l}\text { Pt NPs-PdS NPs } \\
\text { (Pt: N/A, } \\
\text { Pd: N/A) }\end{array}$ & $\mathrm{N} / \mathrm{A}$ & HER & $\mathrm{AQY}=89.0 \%($ at $420 \mathrm{~nm})$ & [290] \\
\hline Hollow-CdS & $\begin{array}{c}\mathrm{MoS}_{2} \text { NPs } \\
\text {-PdS NPs } \\
\text { (Mo: } 7.5, \text { Pd: } 1.5)\end{array}$ & $\mathrm{N} / \mathrm{A}$ & HER & $\mathrm{AQY}=20.9 \%$ (at $495 \mathrm{~nm})$ & [293] \\
\hline Hollow-g- $\mathrm{C}_{3} \mathrm{~N}_{4}$ & $\begin{array}{l}\mathrm{Pt} / \mathrm{Co}_{3} \mathrm{O}_{4} \\
(1.0,3.0)\end{array}$ & $\begin{array}{c}\text { Pt: } 3.0 \\
\mathrm{Co}_{3} \mathrm{O}_{4}: 2.0\end{array}$ & OWSR & $\begin{array}{c}\text { ER }\left(\mathrm{H}_{2}\right)=3.1 \mu \mathrm{mol} \mathrm{h}^{-1} \\
(>300 \mathrm{~nm}) \\
\operatorname{ER~}\left(\mathrm{O}_{2}\right)=1.5 \mu \mathrm{mol} \mathrm{h}{ }^{-1} \\
(>300 \mathrm{~nm})\end{array}$ & [292] \\
\hline $\mathrm{SrTiO}_{3}: \mathrm{Al}$ & $\begin{array}{c}\mathrm{Cr}_{2} \mathrm{O}_{3} / \mathrm{Rh} \text { NPs } \\
\text {-CoOOH NPs } \\
(\mathrm{Cr}: 0.1, \mathrm{Rh}: 0.05, \mathrm{Co}: 0.05)\end{array}$ & $\mathrm{N} / \mathrm{A}$ & OWSR & $\begin{array}{c}\mathrm{AQY}=96 \% \\
\text { (at } 350-360 \mathrm{~nm} \text { ) }\end{array}$ & [288] \\
\hline Hollow- $\mathrm{Ta}_{3} \mathrm{~N}_{5}$ & $\begin{array}{c}\mathrm{Pt} \mathrm{NPs} \\
-\mathrm{IrO}_{2} \mathrm{NPs} \\
\left(\mathrm{Pt}: 1.0, \mathrm{IrO}_{2}: 0.025\right)\end{array}$ & $\begin{array}{l}\text { Pt: } 3.0-5.0 \\
\mathrm{IrO}_{2}: \mathrm{N} / \mathrm{A}\end{array}$ & HER & $\begin{aligned} \mathrm{ER}= & \sim 3.1 \mu \mathrm{mol} \mathrm{h} \mathrm{h}^{-1} \\
& (>400 \mathrm{~nm})\end{aligned}$ & [291] \\
\hline
\end{tabular}

${ }^{a}$ For example, Pt(PD) NPs represent Pt NPs loaded by photodeposition. ${ }^{b}$ OER, HER, and OWSR represent the oxygen evolution reaction, hydrogen evolution reaction, and overall water-splitting reaction, respectively. ${ }^{c}$ AQY, and ER, represent the apparent quantum yield, and evolution rate, respectively. 


\subsection{Forming Facets That Both Excited Electrons and Holes Can Easily Reach}

Some crystallized semiconductor photocatalysts might have facets that are more suitable for reduction or oxidation reactions [294]. For example, $m-\mathrm{BiVO}_{4}$ has a decahedral structure consisting of (010) and (110) facets, where reduction reactions selectively occur on the (010) facets and oxidation reactions on the (110) facets. In 2013, Li, Zhang, and co-workers succeeded in selectively loading Pt NPs as an HER cocatalyst on the (010) facet and $\mathrm{MnO}_{x}(\mathrm{PD}) \mathrm{NPs}$ as an OER cocatalyst on the (110) facet using the photodeposition method (Figure 34A) [287]. As-obtained Pt(PD) NPs-MnO $\mathrm{Mn}_{x}(\mathrm{PD}) \mathrm{NPs} / \mathrm{BiVO}_{4}$ showed a much higher OER rate compared with the photocatalyst with cocatalysts randomly loaded using the impregnation method (Figure 34B). The high OER rate of $\mathrm{Pt}(\mathrm{PD}) \mathrm{NPs}-\mathrm{MnO}_{x}(\mathrm{PD})$ $\mathrm{NPs} / \mathrm{BiVO}_{4}$ obtained using the photodeposition method was attributed to the excited electrons and holes being transferred to different crystal facets and separated efficiently (Figure 34C) [295]. In 2017, Fan, Dittrich, Li, and co-workers also showed that the migration direction of electrons and holes strongly depended on the built-in electric field present in the space charge region of each facet; therefore, controlling the shape of photocatalytic particles and increasing the difference in the internal electric field between each facet can enhance the separation of electrons and holes (Figure 34D) [296]. These authors also found that an asymmetrical array of cocatalysts further enhances the difference in the built-in electric field at the surface (Figure 34D).

The flux method is extremely effective for the synthesis of photocatalysts with such specific facets. In the flux method, raw powder is heated and dissolved in the flux, and then crystals are precipitated by the increase in supersaturation caused by cooling and evaporation of the flux. This method has been used in the synthesis of metal oxides such as $\mathrm{K}_{4} \mathrm{Nb}_{6} \mathrm{O}_{17}\left(\mathrm{~K}=\right.$ potassium) [297-300], $\mathrm{KNb}_{3} \mathrm{O}_{8}$ [301,302], $\mathrm{Na}_{2} \mathrm{Ti}_{6} \mathrm{O}_{13}$ [303-305], and $\mathrm{SnNb}_{2} \mathrm{O}_{6}$ [306,307]. In 2020, Takata, Domen, and co-workers controlled the particle shape of $\mathrm{SrTiO}_{3}: \mathrm{Al}$ using a flux method and subsequently successfully loaded HER and OER cocatalysts selectively onto specific crystal surfaces of $\mathrm{SrTiO}_{3}$ : $\mathrm{Al}$ particles (Figure 35A) [288] In the structure, the photoexcited electrons and holes were selectively transferred to the HER and OER cocatalysts, respectively, and the recombination of electrons and holes, which caused the QY to decrease in conventional photocatalysts, was almost completely suppressed. Therefore, this photocatalyst showed an AQY of 96\% (at 350-360 nm) (Figure 35B). In this study, the UV-light-driven semiconductor $\mathrm{SrTiO}_{3}: \mathrm{Al}$ was used as a water-splitting photocatalyst. In future studies, such a method is expected to be applied to create highly functional Vis-light-driven water-splitting photocatalysts.

\subsection{Formation of One-Dimensional (1D) Nanostructures}

In some cases, the formation of 1D nanostructures can efficiently separate electrons and holes spatially. In 2019, Li and co-workers successfully loaded Pt NPs (HER cocatalyst) on the tip and PdS NPs (OER cocatalyst) on the side of CdSe 1D nanorods (CdSe(1DNRs)) by photodeposition (Pt NPs-PdS NPs/CdSe(1D-NRs); Figure 36A) [289]. The asobtained Pt NPs-PdS NPs/CdSe(1D-NRs) photocatalyst showed a HER activity more than 20 times higher than that of the conventional Pt NPs-PdS NPs/CdSe NPs photocatalyst $(\mathrm{AQY}=\sim 45 \%$ (at $420 \mathrm{~nm})$; Figure 36B). PL measurements showed that these excellent photocatalytic performances were attributed to the decrease in exciton recombination in the CdSe(1D-NRs) caused by the spatial separation of the cocatalysts (Figure 36C). In this photocatalyst, the excited electrons migrate along the long axis to the tip, while the holes migrate to the sides, causing the effective separation of electrons and holes. 
(A)
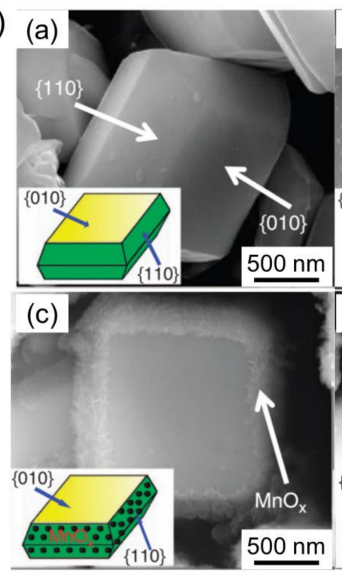

(C)

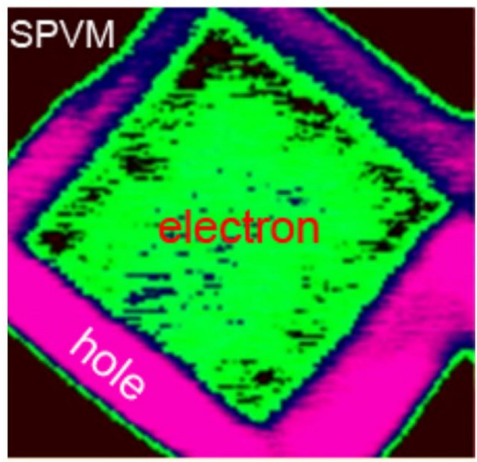

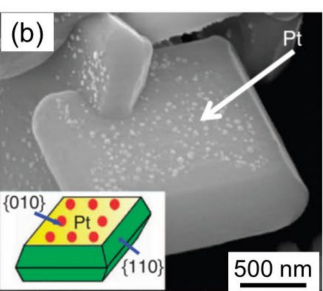

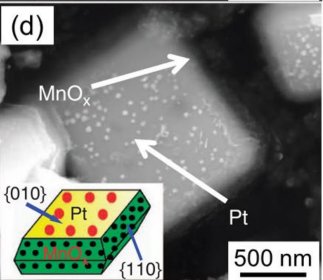

(D) (a)

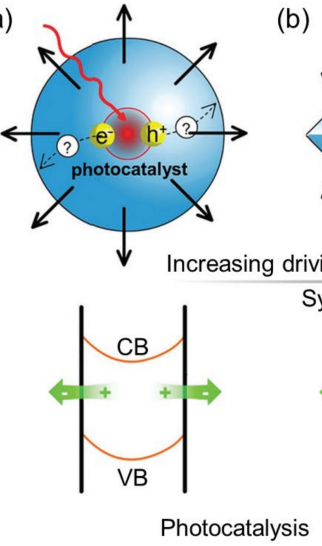

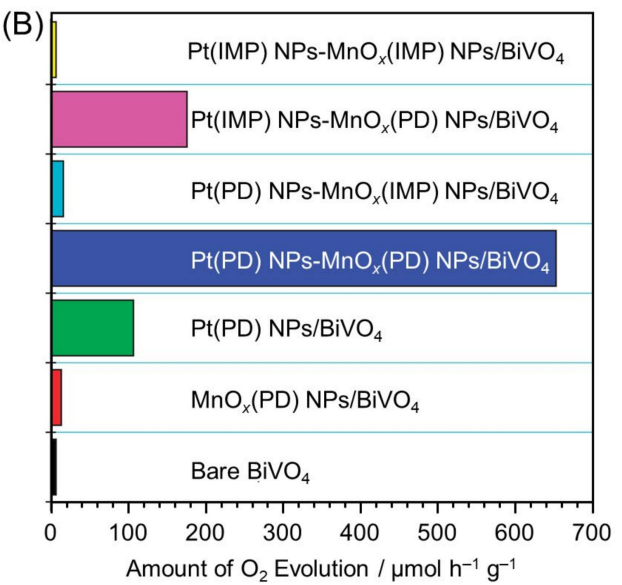

(b)

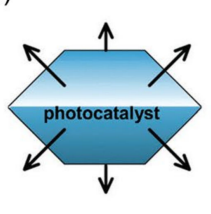

(c)
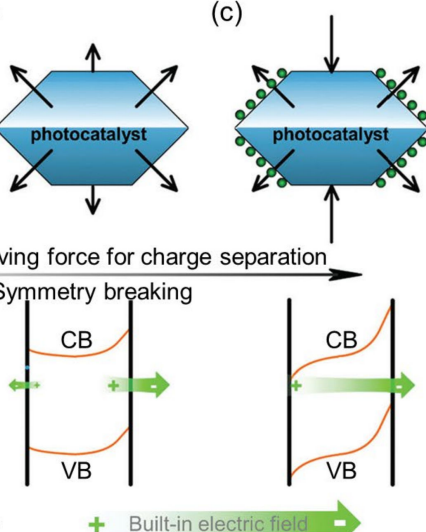

Figure 34. (A) SEM images of $\mathrm{BiVO}_{4}$ with and without metal/metal oxide NPs. (a) Bare $\mathrm{BiVO}_{4}$, (b) $\mathrm{Pt}(\mathrm{PD}) \mathrm{NPs} / \mathrm{BiVO}_{4}$, (c) $\mathrm{MnO}_{x}(\mathrm{PD}) \mathrm{NPs} / \mathrm{BiVO}_{4}$, and (d) $\mathrm{Pt}(\mathrm{PD}) \mathrm{NPs}-\mathrm{MnO}_{x}(\mathrm{PD}) \mathrm{NPs} / \mathrm{BiVO}_{4}$. Contents of the deposited metals/metal oxides are all $5 \mathrm{wt} \%$. (B) Amount of $\mathrm{O}_{2}$ evolved over each photocatalyst. Reaction conditions: $\mathrm{NaIO}_{3}$ aqueous solution $(150 \mathrm{~mL}, 0.02 \mathrm{M})$, Xe lamp (300 W, $\lambda \geq 420 \mathrm{~nm}$ ), top irradiation, reaction time of $1 \mathrm{~h}$. (IMP, impregnation method; PD, photodeposition method; contents of the deposited cocatalysts are all $0.1 \mathrm{wt} \%$ ). (C) Spatial distribution of the surface photovoltage signals. Pink and green colors correspond to holes and electrons separated toward the external surface, respectively. (D) Schematic illustrations showing the driving force for charge separation in photocatalyst particles with (a) symmetric built-in electric fields, (b) anisotropic builtin electric fields on different facets, and (c) an asymmetric cocatalyst assembly. Reproduced with permission from references $[287,295,296]$. Copyright 2013 Springer Nature Limited, 2017 American Chemical Society, and 2018 The Royal Society of Chemistry.

Furthermore, in 2020, $\mathrm{Li}$ and co-workers reported that P-doping the surface of the $\mathrm{Cd}_{0.5} \mathrm{Zn}_{0.5} \mathrm{~S}$ (1D-NRs) photocatalyst $\left(\mathrm{Cd}_{0.5} \mathrm{Zn}_{0.5} \mathrm{~S}\right.$ (1D-NRs):P) enhances electron and hole transfer, producing a Vis-light-driven water-splitting photocatalyst with a high QY [290]. These authors successfully improved the HER activity of the $\mathrm{Cd}_{0.5} \mathrm{Zn}_{0.5} \mathrm{~S}$ (1D-NRs) photocatalyst under Vis-light irradiation by more than two orders of magnitude. They also succeeded in improving the AQY to $89 \%$ (at $420 \mathrm{~nm}$ ) by loading Pt NPs and PdS NPs cocatalysts on the tips and sides of the obtained $\mathrm{Cd}_{0.5} \mathrm{Zn}_{0.5} \mathrm{~S}: \mathrm{P}(1 \mathrm{D}-\mathrm{NRs})$, respectively (Figure 37 ). 
(A)

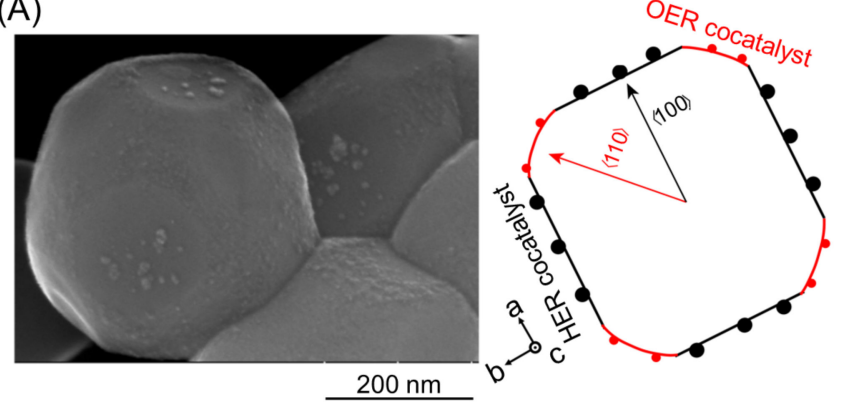

(B)

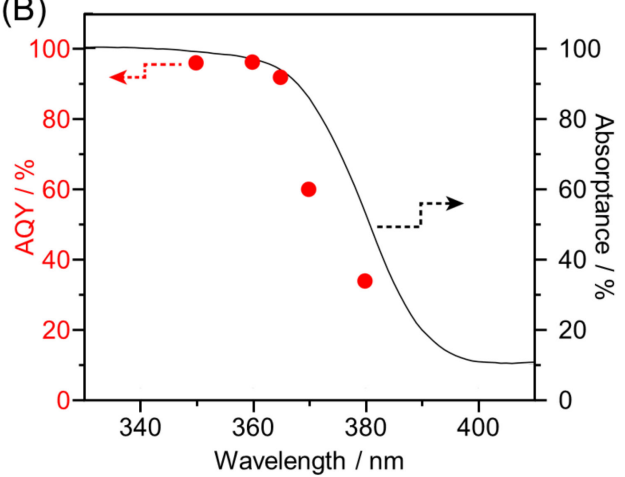

Figure 35. (A) SEM and schematic images of $\mathrm{SrTiO}_{3}$ :Al loaded with various cocatalysts. (B) UV-Vis diffuse reflectance spectrum of bare $\mathrm{SrTiO}_{3}: \mathrm{Al}$ (black solid line) and wavelength dependence of AQY during water splitting on $\mathrm{Cr}_{2} \mathrm{O}_{3}(0.05 \mathrm{wt} \%) / \mathrm{Rh}(0.1 \mathrm{wt} \%) \mathrm{NPs}-\mathrm{CoOOH}(0.05 \mathrm{wt} \%) \mathrm{NPs} / \mathrm{SrTiO}_{3}: \mathrm{Al}$ (red symbols). Reproduced with permission from reference [288]. Copyright 2020 Springer Nature Limited.
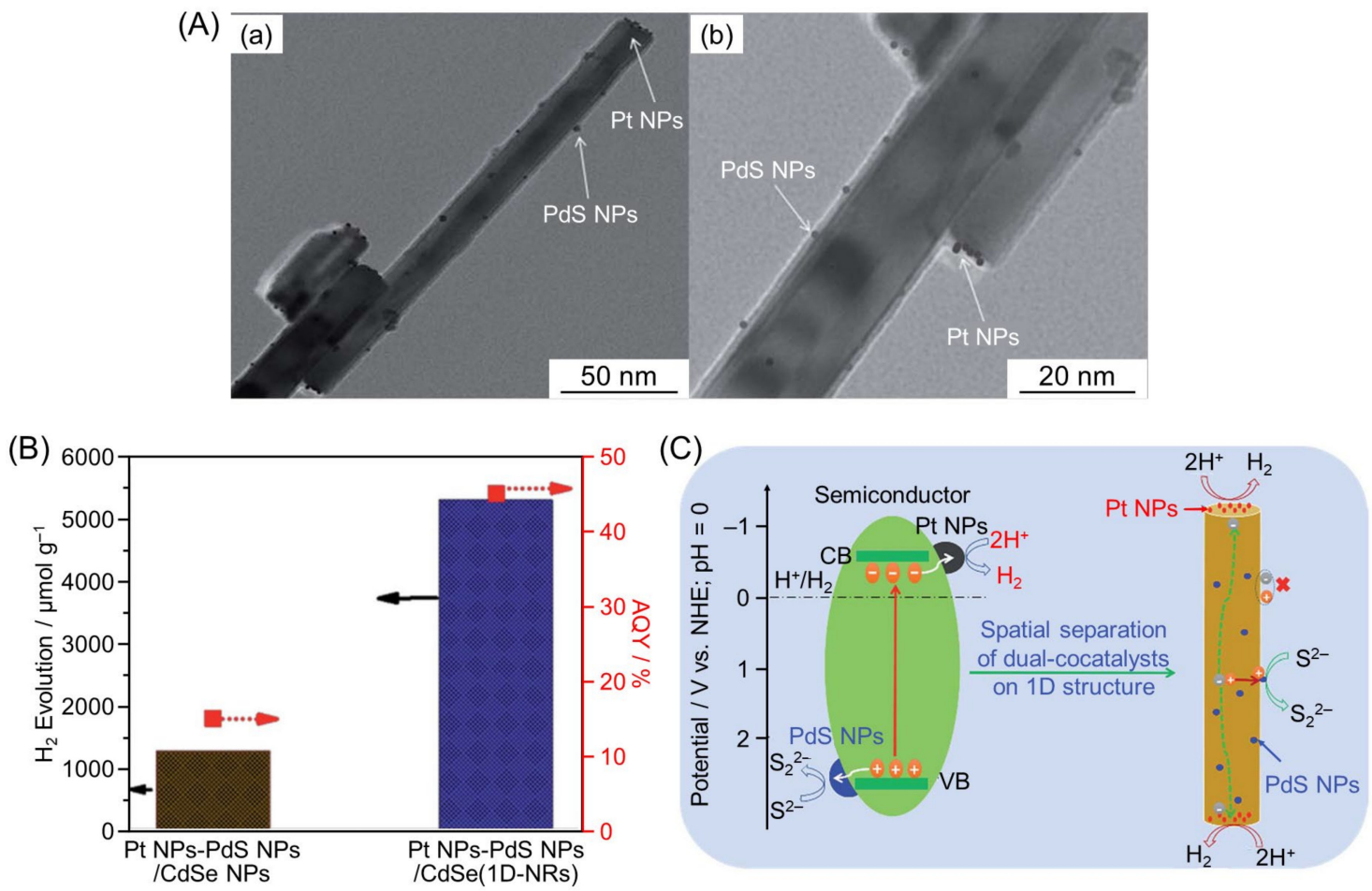

Figure 36. (A) TEM images of Pt NPs-PdS NPs/CdSe 1D nanorods (CdSe(1D-NRs)). Images (a) and (b) are at different magnifications. (B) Comparative $\mathrm{H}_{2}$ evolution of Pt NPs-PdS NPs/CdSe(1D-NRs) and Pt NPs-PdS NPs/CdSe NPs. Conditions: catalyst (100 mg), $\mathrm{Na}_{2} \mathrm{~S}-\mathrm{Na}_{2} \mathrm{SO}_{3}$ aqueous solution $(100 \mathrm{~mL}, 0.1 \mathrm{M})$, and Xe lamp light source $(300 \mathrm{~W})$ with an optical filter $(\lambda>420 \mathrm{~nm})$. (C) Scheme of the proposed mechanism for photocatalytic $\mathrm{H}_{2}$ evolution on CdSe(1D-NRs) with spatial separation of dual cocatalysts. Reproduced with permission from reference [289]. Copyright 2019 The Royal Society of Chemistry. 
(A)

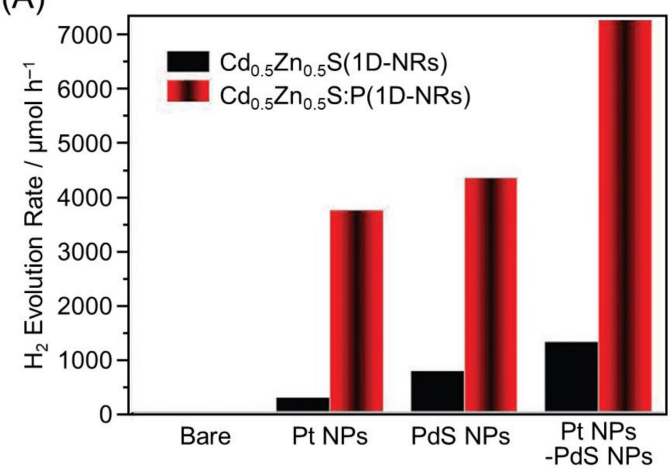

(B)

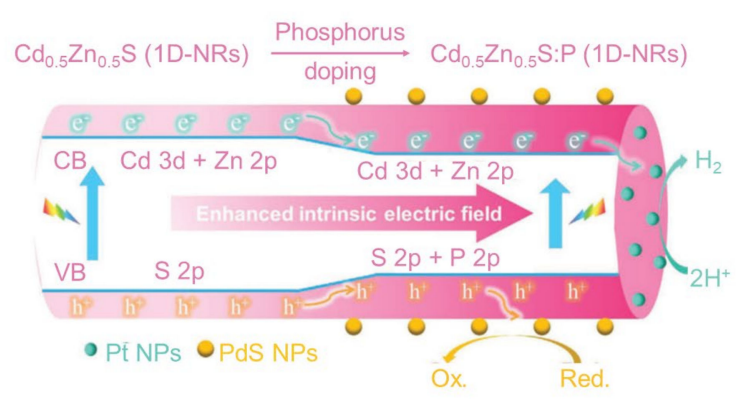

Figure 37. (A) Photocatalytic $\mathrm{H}_{2}$ evolution performance of different samples. Conditions: catalyst, $0.1 \mathrm{~g} ; 0.1 \mathrm{M}$ of $\mathrm{Na}_{2} \mathrm{~S}_{-} \mathrm{Na}_{2} \mathrm{SO}_{3}$ aqueous solution, $100 \mathrm{~mL}$; Xe lamp light source ( $300 \mathrm{~W}$ ) with an optical filter $(\lambda>420 \mathrm{~nm})$. (B) Proposed photogenerated electron-hole transfer mechanism for P-doped CZS NRs to induce a localized intrinsic electric field for spatial separation of redox cocatalysts. Reproduced with permission from reference [290]. Copyright 2020 Wiley-VCH.

\subsection{Formation of Yolk-Shell (Hollow) Nanostructure}

Photocatalysts with suppressed recombination and reverse reactions can be developed by loading HER and OER cocatalysts on the inside and outside of the yolk-shell nanostructure, respectively (Figure 33D). Indeed, in 2013, Domen and co-workers succeeded in significantly enhancing the HER activity by forming $\mathrm{Ta}_{3} \mathrm{~N}_{5}$ yolk-shell nanostructures using $\mathrm{SiO}_{2}$ as a template and selectively loading Pt NPs (HER cocatalyst) and $\mathrm{IrO}_{2} \mathrm{NPs}$ (OER cocatalyst) onto the inside and outside surfaces, respectively (Figure 38) [291]. In 2016, Wang and co-workers also successfully formed g- $\mathrm{C}_{3} \mathrm{~N}_{4}$ hollow spheres using aminated $\mathrm{SiO}_{2}$ as a template and loaded HER cocatalysts (Pt NPs) and OER cocatalysts $\left(\mathrm{Co}_{3} \mathrm{O}_{4} \mathrm{NPs}\right.$ ) onto the inside and outside surfaces, respectively (Figure 39A,B). The obtained photocatalyst showed overall water splitting with the molar ratio of $\mathrm{H}_{2}$ to $\mathrm{O}_{2}$ of 2:1 under UV-light irradiation (Figure 39C(a)) [292]. When both Pt NPs and $\mathrm{Co}_{3} \mathrm{O}_{4} \mathrm{NPs}$ were loaded onto the outside surface of g- $\mathrm{C}_{3} \mathrm{~N}_{4}$, a decrease in water-splitting activity due to decreased $\mathrm{O}_{2}$ evolution was observed (Figure 39C(b)). This indicated that separately loading each cocatalyst onto the inside and outside of the yolk-shell nanostructure is extremely important to obtain high activity. In 2021, Wang and co-workers also succeeded in creating photocatalysts with HER cocatalysts $\left(\mathrm{MoS}_{2} \mathrm{NPs}\right)$ and OER cocatalysts (PdS NPs) loaded onto the outside and inside of CdS hollow spheres, respectively (Figure 40A) [293]. As exciton recombination was greatly suppressed, the obtained photocatalyst showed 115 times higher HER activity compared with CdS NPs (Figure 40B). 
(A)

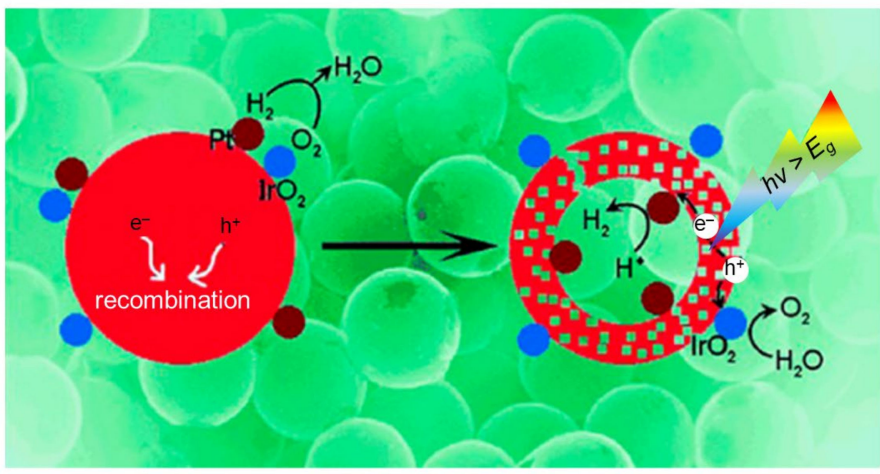

(B)

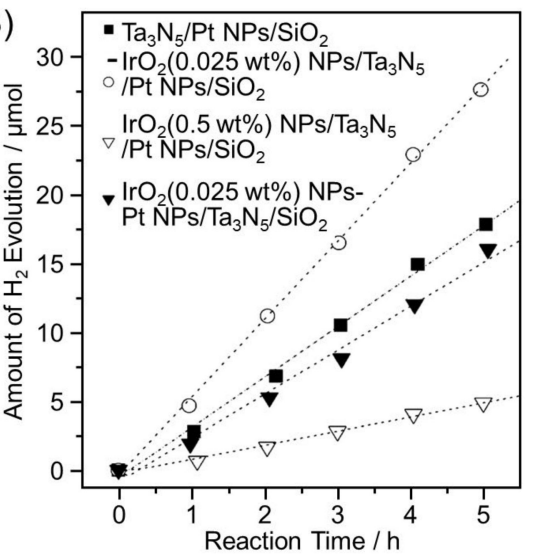

Figure 38. (A) Design principle for a yolk-shell $\mathrm{Ta}_{3} \mathrm{~N}_{5}$ photocatalyst loaded with two separate cocatalysts as effective charge collectors for water splitting. (B) $\mathrm{H}_{2}$-evolution activities on yolk-shell $\mathrm{Ta}_{3} \mathrm{~N}_{5}$ photocatalysts with separated and mixed Pt NPs and $\mathrm{IrO}_{2} \mathrm{NPs}$ cocatalysts. Reaction conditions: $\mathrm{Ta}_{3} \mathrm{~N}_{5}$ catalyst, $0.032 \mathrm{~g}$; methanol, $20 \mathrm{~mL} ; \mathrm{H}_{2} \mathrm{O}, 80 \mathrm{~mL} ; 300 \mathrm{~W}$ Xe lamp with an L42 cutoff filter; top irradiation. Reproduced with permission from reference [291]. Copyright 2013 Wiley-VCH.
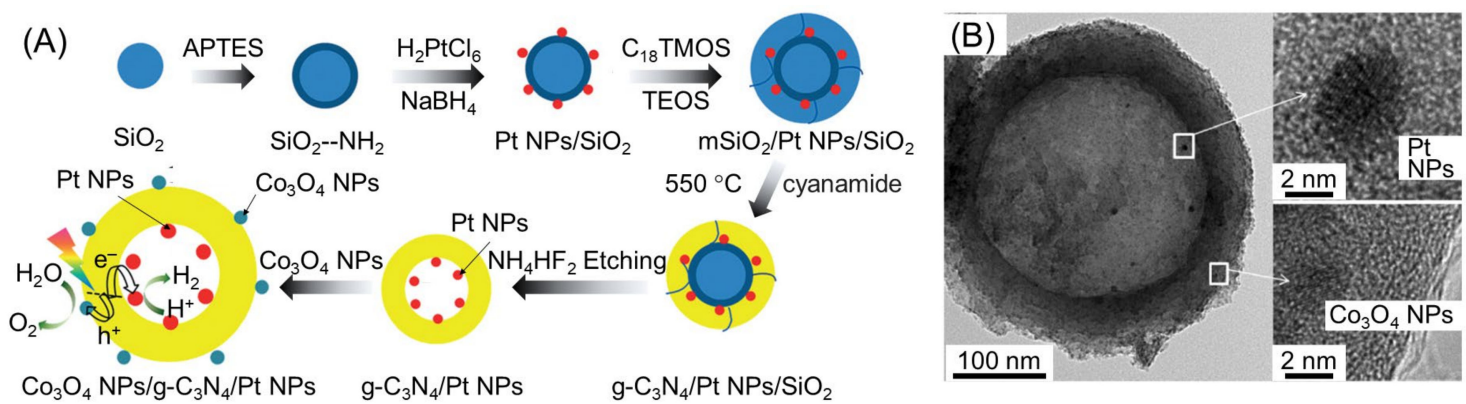

(C)

(a)

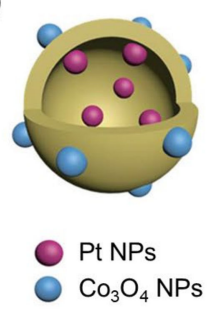

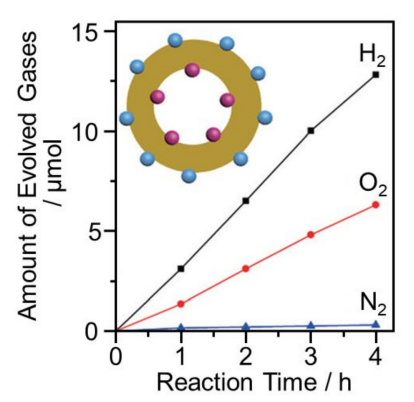

(b)

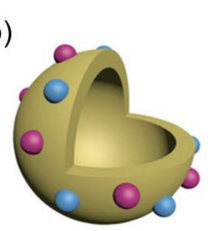

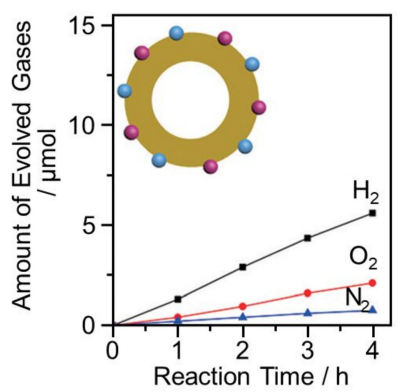

Figure 39. (A) Illustration of the preparation of $\mathrm{Co}_{3} \mathrm{O}_{4} \mathrm{NPs} / \mathrm{g}-\mathrm{C}_{3} \mathrm{~N}_{4} / \mathrm{Pt}$ NPs composites. APTES, C18-TMOS, and TEOS represent 3-aminopropyl triethoxysilane, $n$-octadecyltrimethoxysilane, and tetraethoxysilane, respectively. (B) TEM and HR-TEM images of $\mathrm{Co}_{3} \mathrm{O}_{4} \mathrm{NPs} / \mathrm{g}-\mathrm{C}_{3} \mathrm{~N}_{4} / \mathrm{Pt} \mathrm{NPs}$ samples. (C) Time courses of photocatalytic evolution of $\mathrm{H}_{2}$ and $\mathrm{O}_{2}$ over $(\mathbf{a}) \mathrm{Co}_{3} \mathrm{O}_{4} \mathrm{NPs} / \mathrm{g}-\mathrm{C}_{3} \mathrm{~N}_{4} / \mathrm{Pt} \mathrm{NPs}$ and (b) $\mathrm{Co}_{3} \mathrm{O}_{4} \mathrm{NPs}-\mathrm{Pt} \mathrm{NPs} / \mathrm{g}-\mathrm{C}_{3} \mathrm{~N}_{4}$ under UV-light irradiation $(\lambda>300 \mathrm{~nm})$. Reproduced with permission from reference [292]. Copyright 2016 Wiley-VCH. 
(A)

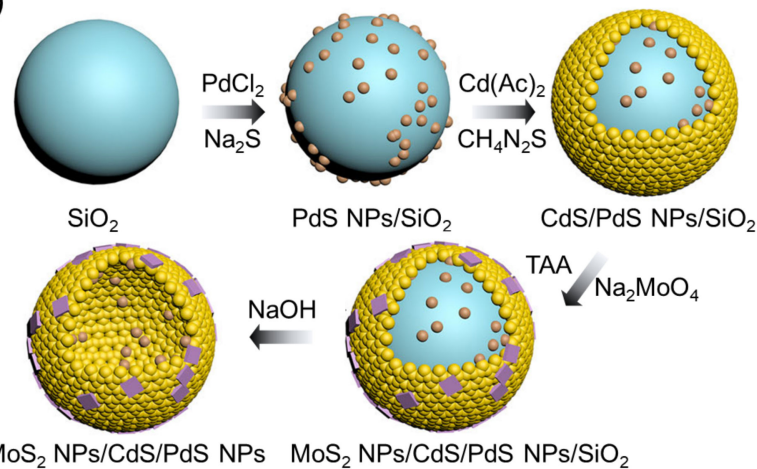

(B)

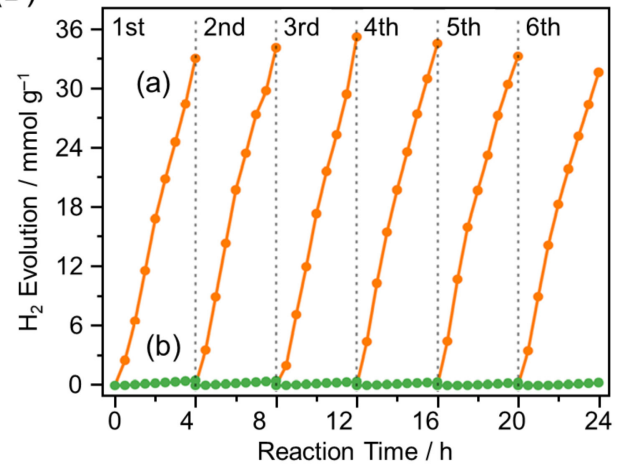

Figure 40. (A) Schematic illustration of the synthetic process for $\mathrm{MoS}_{2}$ NPs/CdS/PdS NPs heterostructures. (B) Cycle stability of $\mathrm{H}_{2}$ evolution from $0.1 \mathrm{M}$ of $\mathrm{Na}_{2} \mathrm{~S}-\mathrm{Na}_{2} \mathrm{SO}_{3}$ aqueous solution under Vis-light irradiation (420-780 nm) using (a) $\mathrm{MoS}_{2}(7.5 \mathrm{wt} \%$ ) NPs/CdS/PdS(1.5 wt $\%$ ) NPs hollow spheres and (b) CdS hollow spheres (each cycle is $4 \mathrm{~h}$ ). Reproduced with permission from reference [293]. Copyright 2021 Wiley-VCH.

\subsection{Formation of Built-in Electric Field in Band Structure}

In 2018, Mi and co-workers proposed a photochemical diode structure in which the excitons generated by photoexcitation were directed to different active sites (Figure 41A) [155]. The proposed structure consisted of vertically aligned InGaN nanowires, with the active sites of HER and OER clearly defined. Photogenerated electrons and holes were instantly separated by the electric field formed perpendicularly to the nanosheet (Figure 41A), which suppressed recombination and reverse reactions at the surface and in the bulk. Specifically, the authors introduced an in-built electric field along the lateral direction of the nanowires by varying the amount of $\mathrm{Mg}$ doping (Figure 41B). Photoexcited electrons transferred to the less Mg-doped side and proceeded with HER, while holes transferred to the more $\mathrm{Mg}$ doped side and proceeded with OER (Figure $41 \mathrm{C}$ ). When the HER cocatalyst $\left(\mathrm{Cr}_{2} \mathrm{O}_{3} / \mathrm{Rh}\right.$ NPs) and OER cocatalyst $\left(\mathrm{CoO}_{x}\right.$ NPs) were loaded on this photocatalyst, the STH reached $5.2 \%$ (Table 2) [141].

(A)

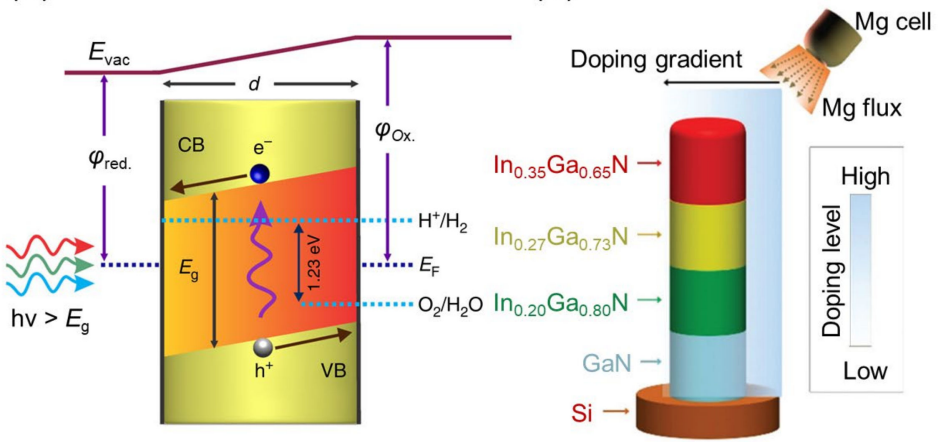

(C)

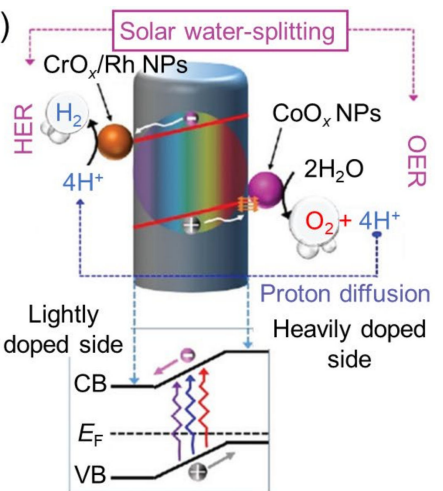

Figure 41. (A) Structural and optical properties of InGaN photochemical diode. Energy-band representation of the proposed photochemical diode (PCD) with radial thickness " $d$ " showing the built-in electric field (band-bending) that separates the excitons (electron and hole) and drives them towards the opposite cathode and anode surfaces. In contrast to a conventional $\mathrm{p}-\mathrm{n}$ PCD, only a single photon absorption is required to generate one active electron-hole pair to participate in the redox reaction (such as a Schottky-type photochemical diode). (B) Schematic of the quadruple-band InGaN nanowire. The p-type dopant originating from the tilted $\mathrm{Mg}$ effusion cell (relative to the nanowire orientation) leads to the $\mathrm{Mg}$-doping gradient profile in the lateral direction of the nanowire. (C) The InGaN nanowire can introduce a built-in electric field for efficient charge carrier separation and extraction for water redox reactions. Reproduced with permission from references [141,155]. Copyright 2018 Springer Nature Limited and 2019 The Royal Society of Chemistry. 


\section{Summary}

This review summarized representative studies of Vis-light-driven water-splitting photocatalysts. This summary clarified the following points regarding the fabrication and functionalization of Vis-light-driven water-splitting photocatalysts.

(1) To develop Vis-light-driven water-splitting photocatalysts, modifying the band structure of stable metal oxide photocatalysts is effective. The main methods are (i) shifting the energy position of the VBM to the negative-potential side by anion or metal cation substitution, (ii) forming impurity levels in the BG by doping, (iii) narrowing the BG by solid solution, and (iv) shifting the entire band structure to the negative-potential side by a reduction in the size of semiconductor particles.

(2) Vis-light-driven water-splitting photocatalysts can also be created by (i) forming multiband-InGaN nanowires, (ii) utilizing $\mathrm{g}-\mathrm{C}_{3} \mathrm{~N}_{4}$, and (iii) forming MOF/COF connections.

(3) Controlling the particle size, chemical composition, morphology, interfacial structure, surface structure, and charge state of the cocatalyst is extremely effective at enhancing the functionality of the photocatalyst.

(4) Forming crystal facets on the photocatalyst surface that excited electrons and holes can easily reach, one-dimensional NR structures, yolk-shell structures, and a built-in electric field in the band structure effectively promote charge separation and suppress recombination, resulting in a high QY.

Sharing these findings with readers is expected to further accelerate the development and practical application of Vis-light-driven water-splitting photocatalysts.

\section{Outlook}

For the practical application of Vis-light-driven water-splitting photocatalysts, much effort is expected to be devoted to the following research areas:

(1) Identifying a simple synthesis method for Vis-light-driven water-splitting photocatalysts. Although several materials have been developed for Vis-light-driven watersplitting photocatalysts, metal (oxy)nitrides and metal (oxy)sulfides are the most attractive materials for OWSR in terms of QY. However, synthesis methods for these photocatalysts have been established only under specific atmospheres. Therefore, only a few research groups with synthesis experience have been able to study these photocatalysts. In the future, simpler methods for synthesizing metal(oxy)nitrides and metal(oxy)sulfide water-splitting photocatalysts are expected to be developed. If realized, more research groups will be able to participate in research on the functionalization of these materials, which is expected to bring their practical application closer to fruition.

(2) Enhancement of exciton separation efficiency. To obtain highly active water-splitting photocatalysts, the exciton separation efficiency must be enhanced [288]. In future, fluorescence lifetime and transient absorption spectroscopic measurements [308-314] are expected to be conducted for a number of photocatalysts to gain a deeper understanding of the influence of the photocatalyst substrate, cocatalyst, and interfacial structure between them on the charge separation efficiency.

(3) Structural analysis of loaded cocatalysts. In order to understand structure-property relationships, it is essential to gain a deeper understanding of the geometric structure of the loaded cocatalysts, especially during the reaction. Therefore, in the future, the geometric structure of loaded cocatalysts is expected to be directly observed using aberration-corrected TEM [315] or scanning TEM. Furthermore, the geometric structure observed under electron irradiation in a vacuum is not necessarily the same as the geometric structure during the water-splitting reaction. Therefore, operando measurements using X-ray absorption fine-structure analysis and other techniques [316] are expected to be applied in photocatalyst studies, which will provide a deeper 
understanding of the geometric structure during the water-splitting reaction than available at present.

(4) Theoretical calculation for real system. Theoretical calculations are also useful for developing highly functional water-splitting photocatalysts. Indeed, previous theoretical calculations have clarified the adsorption state of water molecules [317-319], the rate-limiting step of the reaction [320,321], and the exciton transfer process at the interface between water molecules and photocatalysts [322,323]. However, in most of these studies, theoretical calculations have been performed on simplified models of real systems, and simulations have been performed on a timescale $(<1 \mu \mathrm{s})$ shorter than the actual reaction time (10-900 $\mu \mathrm{s})$. In future, calculations are expected to be performed on longer timescales for real systems, which will provide a deeper understanding of the photocatalytic reaction process.

(5) Construction of practical application system. Photocatalysts with an STH exceeding $10 \%$ need to be developed for practical use in water splitting. Furthermore, a system for the social implementation of water-splitting photocatalysts must be constructed simultaneously. Accordingly, Domen and co-workers have recently succeeded in constructing a $\mathrm{H}_{2}$-production system using photocatalytic panels. However, at present, remaining challenges include the fabrication cost of photocatalytic panels and the performance of the module that separates the evolved gases $\left(\mathrm{H}_{2}\right.$ and $\left.\mathrm{O}_{2}\right)[324,325]$. In future, industry-academia collaborative research is anticipated to be conducted more extensively, which will lead to these challenges being overcome.

\begin{abstract}
Author Contributions: Y.N. and T.K. structured this review. T.K. and M.K. wrote Sections 1-6 and compiled the figures and tables. Y.A., D.H. and D.Y. wrote Sections 3, 4 and 6, respectively. Y.N. revised the entire draft before submission. All authors have read and agreed to the published version of the manuscript.

Funding: This work was supported by the Japan Society for the Promotion of Science (JSPS) KAKENHI (grant numbers 20H02698 and 20H02552), Scientific Research on Innovative Areas “Coordination Asymmetry" (grant numbers 17H05385 and 19H04595), Scientific Research on Innovative Areas "Innovations for Light-Energy Conversion" (grant numbers 18H05178 and 20H05115), Scientific Research on Innovative Areas "Hydrogenomics" (grant numbers 21H00027), and the JST Adaptable and Seamless Technology Transfer Program through Target-driven R\&D (A-STEP, grant number JPMJTM20MS). Funding was provided by Nissanken, the Yashima Environment Technology Foundation, and the Yazaki Memorial Foundation for Science and Technology is gratefully acknowledged.
\end{abstract}

Conflicts of Interest: There are no conflict to declare.

\title{
References
}

1. Fujishima, A.; Rao, T.N.; Tryk, D.A. Titanium Dioxide Photocatalysis. J. Photochem. Photobiol. C 2000, 1, 1-21. [CrossRef]

2. Zou, Z.; Ye, J.; Sayama, K.; Arakawa, H. Direct Splitting of Water under Visible Light Irradiation with an Oxide Semiconductor Photocatalyst. Nature 2001, 414, 625-627. [CrossRef] [PubMed]

3. Abe, R.; Sayama, K.; Domen, K.; Arakawa, H. A New Type of Water Splitting System Composed of Two Different TiO 2 Photocatalysts (Anatase, Rutile) and $\mathrm{IO}_{3}{ }^{-} / \mathrm{I}^{-}$Shuttle Redox Mediator. Chem. Phys. Lett. 2001, 344, 339-344. [CrossRef]

4. Sayama, K.; Mukasa, K.; Abe, R.; Abe, Y.; Arakawa, H. A New Photocatalytic Water Splitting System under Visible Light Irradiation Mimicking a Z-Scheme Mechanism in Photosynthesis. J. Photochem. Photobiol. A 2002, 148, 71-77. [CrossRef]

5. Kato, H.; Asakura, K.; Kudo, A. Highly Efficient Water Splitting into $\mathrm{H}_{2}$ and $\mathrm{O}_{2}$ over Lanthanum-Doped $\mathrm{NaTaO}_{3} \mathrm{Photocatalysts}$ with High Crystallinity and Surface Nanostructure. J. Am. Chem. Soc. 2003, 125, 3082-3089. [CrossRef]

6. Higashi, M.; Abe, R.; Teramura, K.; Takata, T.; Ohtani, B.; Domen, K. Two Step Water Splitting into $\mathrm{H}_{2}$ and $\mathrm{O}_{2}$ under Visible Light by $\mathrm{ATaO}_{2} \mathrm{~N}(\mathrm{~A}=\mathrm{Ca}, \mathrm{Sr}, \mathrm{Ba})$ and $\mathrm{WO}_{3}$ with $\mathrm{IO}_{3}^{-} / \mathrm{I}^{-}$Shuttle Redox Mediator. Chem. Phys. Lett. 2008, 452, 120-123. [CrossRef]

7. Higashi, M.; Abe, R.; Takata, T.; Domen, K. Photocatalytic Overall Water Splitting under Visible Light Using $\mathrm{ATaO} 2 \mathrm{~N}(\mathrm{~A}=\mathrm{Ca}, \mathrm{Sr}$, $\mathrm{Ba}$ ) and $\mathrm{WO}_{3}$ in $\mathrm{IO}_{3}{ }^{-} / \mathrm{I}^{-}$Shuttle Redox Mediated System. Chem. Mater. 2009, 21, 1543-1549. [CrossRef]

8. Takata, T.; Domen, K. Defect Engineering of Photocatalysts by Doping of Aliovalent Metal Cations for Efficient Water Splitting. J. Phys. Chem. C 2009, 113, 19386-19388. [CrossRef]

9. Yi, Z.; Ye, J.; Kikugawa, N.; Kako, T.; Ouyang, S.; Stuart-Williams, H.; Yang, H.; Cao, J.; Luo, W.; Li, Z.; et al. An Orthophosphate Semiconductor with Photooxidation Properties under Visible-Light Irradiation. Nat. Mater. 2010, 9, 559-564. [CrossRef] 
10. Hisatomi, T.; Miyazaki, K.; Takanabe, K.; Maeda, K.; Kubota, J.; Sakata, Y.; Domen, K. Isotopic and Kinetic Assessment of Photocatalytic Water Splitting on $\mathrm{Zn}$-Added $\mathrm{Ga}_{2} \mathrm{O}_{3}$ Photocatalyst Loaded with $\mathrm{Rh}_{2-y} \mathrm{Cr}_{y} \mathrm{O}_{3}$ Cocatalyst. Chem. Phys. Lett. 2010, 486, 144-146. [CrossRef]

11. Iwase, A.; Ng, Y.H.; Ishiguro, Y.; Kudo, A.; Amal, R. Reduced Graphene Oxide as a Solid-State Electron Mediator in Z-Scheme Photocatalytic Water Splitting under Visible Light. J. Am. Chem. Soc. 2011, 133, 11054-11057. [CrossRef]

12. Chen, X.; Liu, L.; Yu, P.Y.; Mao, S.S. Increasing Solar Absorption for Photocatalysis with Black Hydrogenated Titanium Dioxide Nanocrystals. Science 2011, 331, 746-750. [CrossRef] [PubMed]

13. Minegishi, T.; Nishimura, N.; Kubota, J.; Domen, K. Photoelectrochemical Properties of $\mathrm{LaTiO}_{2} \mathrm{~N}$ Electrodes Prepared by Particle Transfer for Sunlight-Driven Water Splitting. Chem. Sci. 2013, 4, 1120-1124. [CrossRef]

14. Iwase, A.; Yoshino, S.; Takayama, T.; Ng, Y.H.; Amal, R.; Kudo, A. Water Splitting and $\mathrm{CO}_{2}$ Reduction under Visible Light Irradiation Using Z-Scheme Systems Consisting of Metal Sulfides, $\mathrm{CoO}_{x}$-Loaded $\mathrm{BiVO}_{4}$, and a Reduced Graphene Oxide Electron Mediator. J. Am. Chem. Soc. 2016, 138, 10260-10264. [CrossRef] [PubMed]

15. Kuriki, R.; Ranasinghe, C.S.K.; Yamazaki, Y.; Yamakata, A.; Ishitani, O.; Maeda, K. Excited-State Dynamics of Graphitic Carbon Nitride Photocatalyst and Ultrafast Electron Injection to a Ru(II) Mononuclear Complex for Carbon Dioxide Reduction. J. Phys. Chem. C 2018, 122, 16795-16802. [CrossRef]

16. Suzuki, T.M.; Yoshino, S.; Takayama, T.; Iwase, A.; Kudo, A.; Morikawa, T. Z-Schematic and Visible-Light-Driven $\mathrm{CO}_{2}$ Reduction

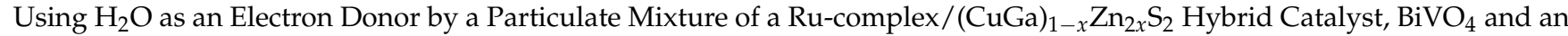
Electron Mediator. Chem. Commun. 2018, 54, 10199-10202. [CrossRef]

17. Nakada, A.; Saeki, A.; Higashi, M.; Kageyama, H.; Abe, R. Two-Step Synthesis of Sillén-Aurivillius Type Oxychlorides to Enhance Their Photocatalytic Activity for Visible-Light-Induced Water Splitting. J. Mater. Chem. A 2018, 6, 10909-10917. [CrossRef]

18. Wakayama, H.; Hibino, K.; Fujii, K.; Oshima, T.; Yanagisawa, K.; Kobayashi, Y.; Kimoto, K.; Yashima, M.; Maeda, K. Synthesis of a Layered Niobium Oxynitride, $\mathrm{Rb}_{2} \mathrm{NdNb}_{2} \mathrm{O}_{6} \mathrm{~N} \cdot \mathrm{H}_{2} \mathrm{O}$, Showing Visible-Light Photocatalytic Activity for $\mathrm{H}_{2}$ Evolution. Inorg. Chem. 2019, 58, 6161-6166. [CrossRef] [PubMed]

19. Luo, Y.; Wang, Z.; Suzuki, S.; Yubuta, K.; Kariya, N.; Hisatomi, T.; Domen, K.; Teshima, K. Fabrication of Single-Crystalline $\mathrm{BaTaO}_{2} \mathrm{~N}$ from Chloride Fluxes for Photocatalytic $\mathrm{H}_{2}$ Evolution under Visible Light. Cryst. Growth Des. 2020, $20,255-261$. [CrossRef]

20. Oshima, T.; Nishioka, S.; Kikuchi, Y.; Hirai, S.; Yanagisawa, K.-I.; Eguchi, M.; Miseki, Y.; Yokoi, T.; Yui, T.; Kimoto, K.; et al. An Artificial Z-Scheme Constructed from Dye-Sensitized Metal Oxide Nanosheets for Visible Light-Driven Overall Water Splitting. J. Am. Chem. Soc. 2020, 142, 8412-8420. [CrossRef]

21. Yoshino, S.; Iwase, A.; Ng, Y.H.; Amal, R.; Kudo, A. Z-Schematic Solar Water Splitting Using Fine Particles of $\mathrm{H}_{2}$-Evolving $(\mathrm{CuGa})_{0.5} \mathrm{ZnS}_{2}$ Photocatalyst Prepared by a Flux Method with Chloride Salts. ACS Appl. Energy Mater. 2020, 3, 5684-5692. [CrossRef]

22. Ho-Kimura, S.; Soontornchaiyakul, W.; Yamaguchi, Y.; Kudo, A. Preparation of Nanoparticle Porous-Structured BiVO $4 \mathrm{Photoan}^{-}$ odes by a New Two-Step Electrochemical Deposition Method for Water Splitting. Catalysts 2021, 11, 136. [CrossRef]

23. Cheng, C.; He, B.; Fan, J.; Cheng, B.; Cao, S.; Yu, J. An Inorganic/Organic S-Scheme Heterojunction $\mathrm{H}_{2}$-Production Photocatalyst and its Charge Transfer Mechanism. Adv. Mater. 2021, 33, 2100317. [CrossRef]

24. Wang, Q.; Hisatomi, T.; Jia, Q.; Tokudome, H.; Zhong, M.; Wang, C.; Pan, Z.; Takata, T.; Nakabayashi, M.; Shibata, N.; et al. Scalable Water Splitting on Particulate Photocatalyst Sheets with a Solar-To-Hydrogen Energy Conversion Efficiency Exceeding 1\%. Nat. Mater. 2016, 15, 611-615. [CrossRef] [PubMed]

25. Ikeda, S.; Fubuki, M.; Takahara, Y.K.; Matsumura, M. Photocatalytic Activity of Hydrothermally Synthesized Tantalate Pyrochlores for Overall Water Splitting. Appl. Catal. A 2006, 300, 186-190. [CrossRef]

26. Ikeda, S.; Hirao, K.; Ishino, S.; Matsumura, M.; Ohtani, B. Preparation of Platinized Strontium Titanate Covered with Hollow Silica and its Activity for Overall Water Splitting in a Novel Phase-Boundary Photocatalytic System. Catal. Today 2006, 117, 343-349. [CrossRef]

27. Youngblood, W.J.; Lee, S.-H.A.; Maeda, K.; Mallouk, T.E. Visible Light Water Splitting Using Dye-Sensitized Oxide Semiconductors. Acc. Chem. Res. 2009, 42, 1966-1973. [CrossRef] [PubMed]

28. Shirai, K.; Sugimoto, T.; Watanabe, K.; Haruta, M.; Kurata, H.; Matsumoto, Y. Effect of Water Adsorption on Carrier Trapping Dynamics at the Surface of Anatase $\mathrm{TiO}_{2}$ Nanoparticles. Nano Lett. 2016, 16, 1323-1327. [CrossRef]

29. Kameyama, T.; Koyama, S.; Yamamoto, T.; Kuwabata, S.; Torimoto, T. Enhanced Photocatalytic Activity of Zn-Ag-In-S Semiconductor Nanocrystals with a Dumbbell-Shaped Heterostructure. J. Phys. Chem. C 2018, 122, 13705-13715. [CrossRef]

30. Kato, Y.; Yamamoto, M.; Ozawa, A.; Tanabe, T.; Yoshida, T. Preparation of Visible-Light-Responsive Photocatalyst by Dehydronitrization of Gallium Oxide Hydroxide for Hydrogen Evolution from Water. Appl. Catal. B 2019, 250, 112-116. [CrossRef]

31. Hsieh, P.-Y.; Kameyama, T.; Takiyama, T.; Masuoka, K.; Yamamoto, T.; Hsu, Y.-J.; Torimoto, T. Controlling the Visible-Light Driven Photocatalytic Activity of Alloyed ZnSe-AgInSe 2 Quantum Dots for Hydrogen Production. J. Mater. Chem. A 2020, 8, 13142-13149. [CrossRef]

32. Yoda, M.; Takashima, T.; Akiyoshi, K.; Torimoto, T.; Irie, H. Red Light-Inducible Overall Water-Splitting Photocatalyst, GoldInserted Zinc Rhodium Oxide and Bismuth Vanadium Oxide Heterojunction, Connected Using Gold Prepared by Sputtering in Ionic Liquid. J. Chem. Phys. 2020, 153, 014701. [CrossRef] [PubMed] 
33. Sudrajat, H.; Kitta, M.; Ito, R.; Yoshida, T.; Katoh, R.; Ohtani, B.; Ichikuni, N.; Onishi, H. The Role of the Shell in Core-ShellStructured La-Doped $\mathrm{NaTaO}_{3}$ Photocatalysts. Phys. Chem. Chem. Phys. 2021, 23, 8868-8879. [CrossRef] [PubMed]

34. Khan, S.; Je, M.; Ton, N.N.T.; Lei, W.; Taniike, T.; Yanagida, S.; Ogawa, D.; Suzuki, N.; Terashima, C.; Fujishima, A.; et al. C-Doped $\mathrm{ZnS}-\mathrm{ZnO} / \mathrm{Rh}$ Nanosheets as Multijunctioned Photocatalysts for Effective $\mathrm{H}_{2}$ Generation from Pure Water under Solar Simulating light. Appl. Catal. B 2021, 297, 120473. [CrossRef]

35. Miyoshi, A.; Vequizo, J.J.M.; Nishioka, S.; Kato, Y.; Yamamoto, M.; Yamashita, S.; Yokoi, T.; Iwase, A.; Nozawa, S.; Yamakata, A.; et al. Nitrogen/Fluorine-Codoped Rutile Titania as a Stable Oxygen-Evolution Photocatalyst for Solar-Driven Z-Scheme Water Splitting. Sustain. Energy Fuels 2018, 2, 2025-2035. [CrossRef]

36. Cheng, T.; Gao, H.; Liu, G.; Pu, Z.; Wang, S.; Yi, Z.; Wu, X.; Yang, H. Preparation of Core-Shell Heterojunction Photocatalysts by Coating CdS Nanoparticles onto $\mathrm{Bi}_{4} \mathrm{Ti}_{3} \mathrm{O}_{12}$ Hierarchical Microspheres and Their Photocatalytic Removal of Organic Pollutants and $\mathrm{Cr}(\mathrm{VI})$ Ions. Colloids Surf. A 2022, 633, 127918. [CrossRef]

37. Chen, P.; Liu, F.; Ding, H.; Chen, S.; Chen, L.; Li, Y.-J.; Au, C.-T.; Yin, S.-F. Porous Double-Shell CdS@C ${ }_{3} \mathrm{~N}_{4}$ Octahedron Derived by in situ Supramolecular Self-Assembly for Enhanced Photocatalytic Activity. Appl. Catal. B 2019, 252, 33-40. [CrossRef]

38. Tang, N.; Li, Y.; Chen, F.; Han, Z. In situ Fabrication of a Direct Z-Scheme Photocatalyst by Immobilizing CdS Quantum Dots in the Channels of Graphene-Hybridized and Supported Mesoporous Titanium Nanocrystals for High Photocatalytic Performance under Visible Light. RSC Adv. 2018, 8, 42233-42245. [CrossRef]

39. Yang, H. A Short Review on Heterojunction Photocatalysts: Carrier Transfer Behavior and Photocatalytic Mechanisms. Mater. Res. Bull. 2021, 142, 111406. [CrossRef]

40. Li, Y.; Li, M.; Xu, P.; Tang, S.; Liu, C. Efficient Photocatalytic Degradation of Acid Orange 7 over N-Doped Ordered Mesoporous Titania on Carbon Fibers under Visible-light Irradiation Based on Three Synergistic Effects. Appl. Catal. A 2016, 524, 163-172. [CrossRef]

41. Lin, X.; Du, S.; Li, C.; Li, G.; Li, Y.; Chen, F.; Fang, P. Consciously Constructing the Robust NiS/g-C ${ }_{3} \mathrm{~N}_{4} \mathrm{Hybrids}_{\text {for Enhanced }}$ Photocatalytic Hydrogen Evolution. Catal. Lett. 2020, 150, 1898-1908. [CrossRef]

42. Xiao, L.; Zhang, Q.; Chen, P.; Chen, L.; Ding, F.; Tang, J.; Li, Y.-J.; Au, C.-T.; Yin, S.-F. Copper-Mediated Metal-Organic Framework as Efficient Photocatalyst for the Partial Oxidation of Aromatic Alcohols under Visible-Light Irradiation: Synergism of Plasmonic Effect and Schottky Junction. Appl. Catal. B 2019, 248, 380-387. [CrossRef]

43. Fujishima, A.; Honda, K. Electrochemical Photolysis of Water at a Semiconductor Electrode. Nature 1972, 238, 37-38. [CrossRef]

44. Chen, S.; Takata, T.; Domen, K. Particulate Photocatalysts for Overall Water Splitting. Nat. Rev. Mater. 2017, 2, 17050. [CrossRef]

45. Hisatomi, T.; Takanabe, K.; Domen, K. Photocatalytic Water-Splitting Reaction from Catalytic and Kinetic Perspectives. Catal. Lett. 2015, 145, 95-108. [CrossRef]

46. Maeda, K.; Domen, K. Photocatalytic Water Splitting: Recent Progress and Future Challenges. J. Phys. Chem. Lett. 2010, 1 , 2655-2661. [CrossRef]

47. Pinaud, B.A.; Benck, J.D.; Seitz, L.C.; Forman, A.J.; Chen, Z.; Deutsch, T.G.; James, B.D.; Baum, K.N.; Baum, G.N.; Ardo, S.; et al. Technical and Economic Feasibility of Centralized Facilities for Solar Hydrogen Production via Photocatalysis and Photoelectrochemistry. Energy Environ. Sci. 2013, 6, 1983-2002. [CrossRef]

48. Fabian, D.M.; Hu, S.; Singh, N.; Houle, F.A.; Hisatomi, T.; Domen, K.; Osterloh, F.E.; Ardo, S. Particle Suspension Reactors and Materials for Solar-Driven Water Splitting. Energy Environ. Sci. 2015, 8, 2825-2850. [CrossRef]

49. Wang, Z.; Li, C.; Domen, K. Recent Developments in Heterogeneous Photocatalysts for Solar-Driven Overall Water Splitting. Chem. Soc. Rev. 2019, 48, 2109-2125. [CrossRef]

50. Maeda, K. Z-Scheme Water Splitting Using Two Different Semiconductor Photocatalysts. ACS Catal. 2013, 3, 1486-1503. [CrossRef]

51. Wang, Y.; Suzuki, H.; Xie, J.; Tomita, O.; Martin, D.J.; Higashi, M.; Kong, D.; Abe, R.; Tang, J. Mimicking Natural Photosynthesis: Solar to Renewable $\mathrm{H}_{2}$ Fuel Synthesis by Z-Scheme Water Splitting Systems. Chem. Rev. 2018, 118, 5201-5241. [CrossRef] [PubMed]

52. Jin, R.; Zeng, C.; Zhou, M.; Chen, Y. Atomically Precise Colloidal Metal Nanoclusters and Nanoparticles: Fundamentals and Opportunities. Chem. Rev. 2016, 116, 10346-10413. [CrossRef]

53. Kang, X.; Li, Y.; Zhu, M.; Jin, R. Atomically Precise Alloy Nanoclusters: Syntheses, Structures, and Properties. Chem. Soc. Rev. 2020, 49, 6443-6514. [CrossRef]

54. Tsukuda, T.; Häkkinen, H. Protected Metal Clusters: From Fundamentals to Applications, 1st ed.; Elsevier Press: Amsterdam, The Netherlands, 2015; Volume 9, pp. 1-358. ISBN 9780081000861.

55. Du, Y.; Sheng, H.; Astruc, D.; Zhu, M. Atomically Precise Noble Metal Nanoclusters as Efficient Catalysts: A Bridge between Structure and Properties. Chem. Rev. 2020, 120, 526-622. [CrossRef]

56. Konishi, K.; Iwasaki, M.; Shichibu, Y. Phosphine-Ligated Gold Clusters with Core+exo Geometries: Unique Properties and Interactions at the Ligand-Cluster Interface. Acc. Chem. Res. 2018, 51, 3125-3133. [CrossRef] [PubMed]

57. Briant, C.E.; Theobald, B.R.C.; White, J.W.; Bell, L.K.; Mingos, D.M.P.; Welch, A.J. Synthesis and X-ray Structural Characterization of the Centred Icosahedral Gold Cluster Compound $\left[\mathrm{Au}_{13}\left(\mathrm{PMe}_{2} \mathrm{Ph}\right)_{10} \mathrm{Cl}_{2}\right]\left(\mathrm{PF}_{6}\right)_{3}$; the Realization of a Theoretical Prediction. $J$. Chem. Soc. Chem. Commun. 1981, 5, 201-202. [CrossRef]

58. Yu, H.; Rao, B.; Jiang, W.; Yang, S.; Zhu, M. The Photoluminescent Metal Nanoclusters with Atomic Precision. Coord. Chem. Rev. 2019, 378, 595-617. [CrossRef] 
59. Agrachev, M.; Ruzzi, M.; Venzo, A.; Maran, F. Nuclear and Electron Magnetic Resonance Spectroscopies of Atomically Precise Gold Nanoclusters. Acc. Chem. Res. 2019, 52, 44-52. [CrossRef] [PubMed]

60. Kwak, K.; Lee, D. Electrochemistry of Atomically Precise Metal Nanoclusters. Acc. Chem. Res. 2019, 52, 12-22. [CrossRef]

61. Nieto-Ortega, B.; Bürgi, T. Vibrational Properties of Thiolate-Protected Gold Nanoclusters. Acc. Chem. Res. 2018, 51, 2811-2819. [CrossRef] [PubMed]

62. Sakthivel, N.A.; Dass, A. Aromatic Thiolate-Protected Series of Gold Nanomolecules and a Contrary Structural Trend in Size Evolution. Acc. Chem. Res. 2018, 51, 1774-1783. [CrossRef]

63. Whetten, R.L.; Weissker, H.-C.; Pelayo, J.J.; Mullins, S.M.; López-Lozano, X.; Garzón, I.L. Chiral-Icosahedral (I) Symmetry in Ubiquitous Metallic Cluster Compounds (145A,60X): Structure and Bonding Principles. Acc. Chem. Res. 2019, 52, 34-43. [CrossRef] [PubMed]

64. Bhattarai, B.; Zaker, Y.; Atnagulov, A.; Yoon, B.; Landman, U.; Bigioni, T.P. Chemistry and Structure of Silver Molecular Nanoparticles. Acc. Chem. Res. 2018, 51, 3104-3113. [CrossRef]

65. Gan, Z.; Xia, N.; Wu, Z. Discovery, Mechanism, and Application of Antigalvanic Reaction. Acc. Chem. Res. 2018, 51, 2774-2783. [CrossRef] [PubMed]

66. Ghosh, A.; Mohammed, O.F.; Bakr, O.M. Atomic-Level Doping of Metal Clusters. Acc. Chem. Res. 2018, 51, 3094-3103. [CrossRef]

67. Yan, J.; Teo, B.K.; Zheng, N. Surface Chemistry of Atomically Precise Coinage-Metal Nanoclusters: From Structural Control to Surface Reactivity and Catalysis. Acc. Chem. Res. 2018, 51, 3084-3093. [CrossRef]

68. Lee, S.; Bootharaju, M.S.; Deng, G.; Malola, S.; Baek, W.; Häkkinen, H.; Zheng, N.; Hyeon, T. $\left[\mathrm{Cu}_{32}\left(\mathrm{PET}_{24} \mathrm{H}_{8} \mathrm{Cl}_{2}\right]\left(\mathrm{PPh}_{4}\right)_{2}: \mathrm{A}\right.$ Copper Hydride Nanocluster with a Bisquare Antiprismatic Core. J. Am. Chem. Soc. 2020, 142, 13974-13981. [CrossRef]

69. Mitsui, M.; Nagaoka, S.; Matsumoto, T.; Nakajima, A. Soft-Landing Isolation of Vanadium-Benzene Sandwich Clusters on a Room-Temperature Substrate Using n-Alkanethiolate Self-Assembled Monolayer Matrixes. J. Phys. Chem. B 2006, 110, $2968-2971$. [CrossRef]

70. Nakaya, M.; Iwasa, T.; Tsunoyama, H.; Eguchi, T.; Nakajima, A. Formation of a Superatom Monolayer Using Gas-PhaseSynthesized Ta@Si ${ }_{16}$ Nanocluster Ions. Nanoscale 2014, 6, 14702-14707. [CrossRef] [PubMed]

71. Minamitani, E.; Takagi, N.; Arafune, R.; Frederiksen, T.; Komeda, T.; Ueba, H.; Watanabe, S. Inelastic Electron Tunneling Spectroscopy by STM of Phonons at Solid Surfaces and Interfaces. Prog. Surf. Sci. 2018, 93, 131-145. [CrossRef]

72. Iida, K.; Noda, M.; Ishimura, K.; Nobusada, K. First-Principles Computational Visualization of Localized Surface Plasmon Resonance in Gold Nanoclusters. J. Phys. Chem. A 2014, 118, 11317-11322. [CrossRef]

73. Negishi, Y.; Mizuno, M.; Hirayama, M.; Omatoi, M.; Takayama, T.; Iwase, A.; Kudo, A. Enhanced Photocatalytic Water Splitting by $\mathrm{BaLa}_{4} \mathrm{Ti}_{4} \mathrm{O}_{15}$ Loaded with $1 \mathrm{~nm}$ Gold Nanoclusters Using Glutathione-Protected $\mathrm{Au}_{25}$ Clusters. Nanoscale 2013, 5, 7188-7192. [CrossRef] [PubMed]

74. Negishi, Y.; Matsuura, Y.; Tomizawa, R.; Kurashige, W.; Niihori, Y.; Takayama, T.; Iwase, A.; Kudo, A. Controlled Loading of Small $\mathrm{Au}_{n}$ Clusters $(n=10-39)$ onto $\mathrm{BaLa}_{4} \mathrm{Ti}_{4} \mathrm{O}_{15}$ Photocatalysts: Toward an Understanding of Size Effect of Cocatalyst on Water-Splitting Photocatalytic Activity. J. Phys. Chem. C 2015, 119, 11224-11232. [CrossRef]

75. Kurashige, W.; Kumazawa, R.; Ishii, D.; Hayashi, R.; Niihori, Y.; Hossain, S.; Nair, L.V.; Takayama, T.; Iwase, A.; Yamazoe, S.; et al. $\mathrm{Au}_{25}$-Loaded $\mathrm{BaLa}_{4} \mathrm{Ti}_{4} \mathrm{O}_{15}$ Water-Splitting Photocatalyst with Enhanced Activity and Durability Produced Using New Chromium Oxide Shell Formation Method. J. Phys. Chem. C 2018, 122, 13669-13681. [CrossRef]

76. Kurashige, W.; Hayashi, R.; Wakamatsu, K.; Kataoka, Y.; Hossain, S.; Iwase, A.; Kudo, A.; Yamazoe, S.; Negishi, Y. AtomicLevel Understanding of the Effect of Heteroatom Doping of the Cocatalyst on Water-Splitting Activity in AuPd or AuPt Alloy Cluster-Loaded $\mathrm{BaLa}_{4} \mathrm{Ti}_{4} \mathrm{O}_{15}$. ACS Appl. Energy Mater. 2019, 2, 4175-4187. [CrossRef]

77. Kurashige, W.; Mori, Y.; Ozaki, S.; Kawachi, M.; Hossain, S.; Kawawaki, T.; Shearer, C.J.; Iwase, A.; Metha, G.F.; Yamazoe, S.; et al. Activation of Water-Splitting Photocatalysts by Loading with Ultrafine Rh-Cr Mixed-Oxide Cocatalyst Nanoparticles. Angezw. Chem. Int. Ed. 2020, 59, 7076-7082. [CrossRef]

78. Kawawaki, T.; Kataoka, Y.; Hirata, M.; Akinaga, Y.; Takahata, R.; Wakamatsu, K.; Fujiki, Y.; Kataoka, M.; Kikkawa, S.; Alotabi, A.S.; et al. Creation of High-Performance Heterogeneous Photocatalysts by Controlling Ligand Desorption and Particle Size of Gold Nanocluster. Angew. Chem. Int. Ed. 2021, 60, 21340-21350. [CrossRef]

79. Kawawaki, T.; Kataoka, Y.; Ozaki, S.; Kawachi, M.; Hirata, M.; Negishi, Y. Creation of Active Water-Splitting Photocatalysts by Controlling Cocatalysts Using Atomically Precise Metal Nanoclusters. Chem. Commun. 2021, 57, 417-440. [CrossRef]

80. Nakata, K.; Fujishima, A. $\mathrm{TiO}_{2}$ Photocatalysis: Design and Applications. J. Photochem. Photobiol. C 2012, 13, 169-189. [CrossRef]

81. Chen, X.; Shen, S.; Guo, L.; Mao, S.S. Semiconductor-Based Photocatalytic Hydrogen Generation. Chem. Rev. 2010, 110, 6503-6570. [CrossRef]

82. Abe, R. Recent Progress on Photocatalytic and Photoelectrochemical Water Splitting under Visible Light Irradiation. J. Photochem. Photobiol. C 2010, 11, 179-209. [CrossRef]

83. Maeda, K. Photocatalytic Water Splitting Using Semiconductor Particles: History and Recent Developments. J. Photochem. Photobiol. C 2011, 12, 237-268. [CrossRef]

84. Wang, H.; Zhang, L.; Chen, Z.; Hu, J.; Li, S.; Wang, Z.; Liu, J.; Wang, X. Semiconductor Heterojunction Photocatalysts: Design, Construction, and Photocatalytic Performances. Chem. Soc. Rev. 2014, 43, 5234-5244. [CrossRef]

85. Hisatomi, T.; Kubota, J.; Domen, K. Recent Advances in Semiconductors for Photocatalytic and Photoelectrochemical Water Splitting. Chem. Soc. Rev. 2014, 43, 7520-7535. [CrossRef] 
86. Kudo, A.; Miseki, Y. Heterogeneous Photocatalyst Materials for Water Splitting. Chem. Soc. Rev. 2009, 38, 253-278. [CrossRef]

87. Wang, Q.; Domen, K. Particulate Photocatalysts for Light-Driven Water Splitting: Mechanisms, Challenges, and Design Strategies. Chem. Rev. 2020, 120, 919-985. [CrossRef]

88. Kawawaki, T.; Mori, Y.; Wakamatsu, K.; Ozaki, S.; Kawachi, M.; Hossain, S.; Negishi, Y. Controlled Colloidal Metal Nanoparticles and Nanoclusters: Recent Applications as Cocatalysts for Improving Photocatalytic Water-Splitting Activity. J. Mater. Chem. A 2020, 8, 16081-16113. [CrossRef]

89. Maeda, K.; Mallouk, T.E. Two-Dimensional Metal Oxide Nanosheets as Building Blocks for Artificial Photosynthetic Assemblies. Bull. Chem. Soc. Jpn. 2019, 92, 38-54. [CrossRef]

90. Seo, J.; Nishiyama, H.; Yamada, T.; Domen, K. Visible-Light-Responsive Photoanodes for Highly Active, Stable Water Oxidation. Angew. Chem. Int. Ed. 2018, 57, 8396-8415. [CrossRef]

91. Yamaguchi, Y.; Kudo, A. Visible Light Responsive Photocatalysts Developed by Substitution with Metal Cations Aiming at Artificial Photosynthesis. Front. Energy 2021, 15, 568-576. [CrossRef]

92. De Respinis, M.; Fravventura, M.; Abdi, F.F.; Schreuders, H.; Savenije, T.J.; Smith, W.A.; Dam, B.; van de Krol, R. Oxynitrogenography: Controlled Synthesis of Single-Phase Tantalum Oxynitride Photoabsorbers. Chem. Mater. 2015, 27, 7091-7099. [CrossRef]

93. Chun, W.-J.; Ishikawa, A.; Fujisawa, H.; Takata, T.; Kondo, J.N.; Hara, M.; Kawai, M.; Matsumoto, Y.; Domen, K. Conduction and Valence Band Positions of $\mathrm{Ta}_{2} \mathrm{O}_{5}, \mathrm{TaON}$, and $\mathrm{Ta}_{3} \mathrm{~N}_{5}$ by UPS and Electrochemical Methods. J. Phys. Chem. B 2003, 107, 1798-1803. [CrossRef]

94. Hitoki, G.; Takata, T.; Kondo, J.N.; Hara, M.; Kobayashi, H.; Domen, K. An Oxynitride, TaON, as an Efficient Water Oxidation Photocatalyst under Visible Light Irradiation $(\lambda \leq 500 \mathrm{~nm})$. Chem. Commun. 2002, 16, 1698-1699. [CrossRef]

95. Hara, M.; Nunoshige, J.; Takata, T.; Kondo, J.N.; Domen, K. Unusual Enhancement of $\mathrm{H}_{2}$ Evolution by Ru on TaON Photocatalyst under Visible Light Irradiation. Chem. Commun. 2003, 24, 3000-3001. [CrossRef]

96. Maeda, K.; Lu, D.; Domen, K. Direct Water Splitting into Hydrogen and Oxygen under Visible Light by Using Modified TaON Photocatalysts with $\mathrm{d}^{0}$ Electronic Configuration. Chem. Eur. J. 2013, 19, 4986-4991. [CrossRef]

97. Hara, M.; Takata, T.; Kondo, J.N.; Domen, K. Photocatalytic Reduction of Water by TaON under Visible Light Irradiation. Catal. Today 2004, 90, 313-317. [CrossRef]

98. Hitoki, G.; Ishikawa, A.; Takata, T.; Kondo, J.N.; Hara, M.; Domen, K. Ta ${ }_{3} \mathrm{~N}_{5}$ as a Novel Visible Light-Driven Photocatalyst $(\lambda<$ $600 \mathrm{~nm})$. Chem. Lett. 2002, 31, 736-737.

99. Maeda, K.; Domen, K. New Non-Oxide Photocatalysts Designed for Overall Water Splitting under Visible Light. J. Phys. Chem. C 2007, 111, 7851-7861. [CrossRef]

100. Ishikawa, A.; Takata, T.; Kondo, J.N.; Hara, M.; Kobayashi, H.; Domen, K. Oxysulfide $\mathrm{Sm}_{2} \mathrm{Ti}_{2} \mathrm{~S}_{2} \mathrm{O}_{5}$ as a Stable Photocatalyst for Water Oxidation and Reduction under Visible Light Irradiation $(\lambda \leq 650 \mathrm{~nm})$. J. Am. Chem. Soc. 2002, 124, 13547-13553. [CrossRef]

101. Ishikawa, A.; Takata, T.; Matsumura, T.; Kondo, J.N.; Hara, M.; Kobayashi, H.; Domen, K. Oxysulfides $\mathrm{Ln}_{2} \mathrm{Ti}_{2} \mathrm{~S}_{2} \mathrm{O}_{5}$ as $\mathrm{Stable}$ Photocatalysts for Water Oxidation and Reduction under Visible-Light Irradiation. J. Phys. Chem. B 2004, 108, $2637-2642$. [CrossRef]

102. Goto, Y.; Seo, J.; Kumamoto, K.; Hisatomi, T.; Mizuguchi, Y.; Kamihara, Y.; Katayama, M.; Minegishi, T.; Domen, K. Crystal

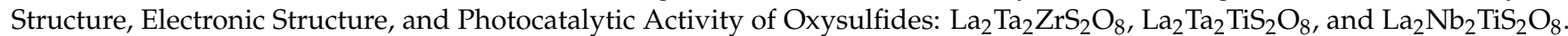
Inorg. Chem. 2016, 55, 3674-3679. [CrossRef]

103. Wang, Q.; Nakabayashi, M.; Hisatomi, T.; Sun, S.; Akiyama, S.; Wang, Z.; Pan, Z.; Xiao, X.; Watanabe, T.; Yamada, T.; et al Oxysulfide Photocatalyst for Visible-Light-Driven Overall Water Splitting. Nat. Mater. 2019, 18, 827-832. [CrossRef] [PubMed]

104. Zhang, F.; Maeda, K.; Takata, T.; Domen, K. Modification of Oxysulfides with Two Nanoparticulate Cocatalysts to Achieve Enhanced Hydrogen Production from Water with Visible Light. Chem. Commun. 2010, 46, 7313-7315. [CrossRef]

105. Sakai, E.; Nagamura, N.; Liu, J.; Hisatomi, T.; Yamada, T.; Domen, K.; Oshima, M. Investigation of the Enhanced Photocathodic Activity of $\mathrm{La}_{5} \mathrm{Ti}_{2} \mathrm{CuS}_{5} \mathrm{O}_{7}$ Photocathodes in $\mathrm{H}_{2}$ Evolution by Synchrotron Radiation Nanospectroscopy. Nanoscale 2016, 8 , 18893-18896. [CrossRef] [PubMed]

106. Nandy, S.; Hisatomi, T.; Ma, G.; Minegishi, T.; Katayama, M.; Domen, K. Enhancement of the $\mathrm{H}_{2}$ Evolution Activity of $\mathrm{La}_{5} \mathrm{Ti}_{2} \mathrm{Cu}\left(\mathrm{S}_{1-x} \mathrm{Se}_{x}\right)_{5} \mathrm{O}_{7}$ Photocatalysts by Coloading Pt and NiS Cocatalysts. J. Mater. Chem. A 2017, 5, 6106-6112. [CrossRef]

107. Shi, R.; Ye, H.-F.; Liang, F.; Wang, Z.; Li, K.; Weng, Y.; Lin, Z.; Fu, W.-F.; Che, C.-M.; Chen, Y. Interstitial P-Doped CdS with Long-Lived Photogenerated Electrons for Photocatalytic Water Splitting without Sacrificial Agents. Adv. Mater. 2018, $30,1705941$. [CrossRef] [PubMed]

108. Xiao, X.; Liu, C.; Hu, R.; Zuo, X.; Nan, J.; Li, L.; Wang, L. Oxygen-Rich Bismuth Oxyhalides: Generalized One-Pot Synthesis, Band Structures and Visible-Light Photocatalytic Properties. J. Mater. Chem. 2012, 22, 22840-22843. [CrossRef]

109. Kato, D.; Hongo, K.; Maezono, R.; Higashi, M.; Kunioku, H.; Yabuuchi, M.; Suzuki, H.; Okajima, H.; Zhong, C.; Nakano, K.; et al. Valence Band Engineering of Layered Bismuth Oxyhalides toward Stable Visible-Light Water Splitting: Madelung Site Potential Analysis. J. Am. Chem. Soc. 2017, 139, 18725-18731. [CrossRef]

110. Fujito, H.; Kunioku, H.; Kato, D.; Suzuki, H.; Higashi, M.; Kageyama, H.; Abe, R. Layered Perovskite Oxychloride Bi ${ }_{4} \mathrm{NbO}_{8} \mathrm{Cl}$ : A Stable Visible Light Responsive Photocatalyst for Water Splitting. J. Am. Chem. Soc. 2016, 138, 2082-2085. [CrossRef]

111. Tao, X.; Zhao, Y.; Mu, L.; Wang, S.; Li, R.; Li, C. Bismuth Tantalum Oxyhalogen: A Promising Candidate Photocatalyst for Solar Water Splitting. Adv. Energy Mater. 2018, 8, 1701392. [CrossRef] 
112. Kudo, A.; Omori, K.; Kato, H. A Novel Aqueous Process for Preparation of Crystal Form-Controlled and Highly Crystalline $\mathrm{BiVO}_{4}$ Powder from Layered Vanadates at Room Temperature and Its Photocatalytic and Photophysical Properties. J. Am. Chem. Soc. 1999, 121, 11459-11467. [CrossRef]

113. Cooper, J.K.; Gul, S.; Toma, F.M.; Chen, L.; Glans, P.-A.; Guo, J.; Ager, J.W.; Yano, J.; Sharp, I.D. Electronic Structure of Monoclinic $\mathrm{BiVO}_{4}$. Chem. Mater. 2014, 26, 5365-5373. [CrossRef]

114. Iwashina, K.; Iwase, A.; Kudo, A. Sensitization of Wide Band Gap Photocatalysts to Visible Light by Molten CuCl Treatment. Chem. Sci. 2015, 6, 687-692. [CrossRef]

115. Watanabe, K.; Iwase, A.; Kudo, A. Solar Water Splitting over $\mathrm{Rh}_{0.5} \mathrm{Cr}_{1.5} \mathrm{O}_{3}$-Loaded $\mathrm{AgTaO}_{3}$ of a Valence-Band-Controlled Metal Oxide Photocatalyst. Chem. Sci. 2020, 11, 2330-2334. [CrossRef]

116. Kaga, H.; Saito, K.; Kudo, A. Solar Hydrogen Production over Novel Metal Sulfide Photocatalysts of $A G a_{2} \mathrm{In}_{3} \mathrm{~S}_{8}(\mathrm{~A}=\mathrm{Cu}$ or $\mathrm{Ag}$ ) with Layered Structures. Chem. Commun. 2010, 46, 3779-3781. [CrossRef]

117. Watanabe, K.; Iwashina, K.; Iwase, A.; Nozawa, S.; Adachi, S.-i.; Kudo, A. New Visible-Light-Driven $\mathrm{H}_{2}-$ and $\mathrm{O}_{2}$-Evolving Photocatalysts Developed by $\mathrm{Ag}(\mathrm{I})$ and $\mathrm{Cu}(\mathrm{I})$ Ion Exchange of Various Layered and Tunneling Metal Oxides Using Molten Salts Treatments. Chem. Mater. 2020, 32, 10524-10537. [CrossRef]

118. Horie, H.; Iwase, A.; Kudo, A. Photocatalytic Properties of Layered Metal Oxides Substituted with Silver by a Molten $\mathrm{AgNO}_{3}$ Treatment. ACS Appl. Mater. Interfaces 2015, 7, 14638-14643. [CrossRef]

119. Konta, R.; Ishii, T.; Kato, H.; Kudo, A. Photocatalytic Activities of Noble Metal Ion Doped $\mathrm{SrTiO}_{3}$ under Visible Light Irradiation. J. Phys. Chem. B 2004, 26, 8992-8995. [CrossRef]

120. Asai, R.; Nemoto, H.; Jia, Q.; Saito, K.; Iwase, A.; Kudo, A. A Visible Light Responsive Rhodium and Antimony-Codoped $\mathrm{SrTiO}_{3}$ Powdered Photocatalyst Loaded with an $\mathrm{IrO}_{2}$ Cocatalyst for Solar Water Splitting. Chem. Commun. 2014, 50, $2543-2546$. [CrossRef]

121. Shinoda, T.; Yamaguchi, Y.; Kudo, A.; Murakami, N. In situ Photoacoustic Analysis of Near-Infrared Absorption of RhodiumDoped Strontium Titanate Photocatalyst Powder. Chem. Commun. 2020, 56, 14255-14258. [CrossRef]

122. Kato, H.; Kudo, A. Visible-Light-Response and Photocatalytic Activities of $\mathrm{TiO}_{2}$ and $\mathrm{SrTiO}_{3}$ Photocatalysts Codoped with Antimony and Chromium. J. Phys. Chem. B 2002, 106, 5029-5034. [CrossRef]

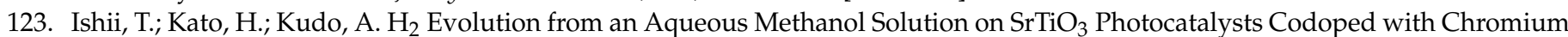
and Tantalum Ions under Visible Light Irradiation. J. Photochem. Photobiol. A 2004, 163, 181-186. [CrossRef]

124. Modak, B.; Ghosh, S.K. Origin of Enhanced Visible Light Driven Water Splitting by (Rh, Sb)-SrTiO 3 . Phys. Chem. Chem. Phys. 2015, 17, 15274-15283. [CrossRef]

125. Furuhashi, K.; Jia, Q.; Kudo, A.; Onishi, H. Time-Resolved Infrared Absorption Study of $\mathrm{SrTiO}_{3}$ Photocatalysts Codoped with Rhodium and Antimony. J. Phys. Chem. C 2013, 117, 19101-19106. [CrossRef]

126. Wang, Q.; Hisatomi, T.; Ma, S.S.K.; Li, Y.; Domen, K. Core/Shell Structured La- and Rh-Codoped SrTiO 3 as a Hydrogen Evolution Photocatalyst in Z-Scheme Overall Water Splitting under Visible Light Irradiation. Chem. Mater. 2014, 26, 4144-4150. [CrossRef]

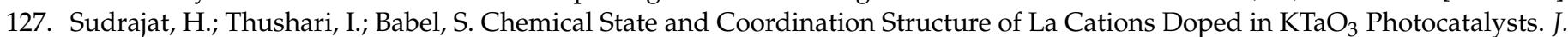
Phys. Chem. Solids 2019, 127, 94-100. [CrossRef]

128. Jo, W.J.; Kang, H.J.; Kong, K.-J.; Lee, Y.S.; Park, H.; Lee, Y.; Buonassisi, T.; Gleason, K.K.; Lee, J.S. Phase Transition-Induced Band Edge Engineering of $\mathrm{BiVO}_{4}$ to Split Pure Water under Visible Light. Proc. Natl. Acad. Sci. USA 2015, 112, 13774-13778. [CrossRef] [PubMed]

129. Maeda, K.; Takata, T.; Hara, M.; Saito, N.; Inoue, Y.; Kobayashi, H.; Domen, K. GaN:ZnO Solid Solution as a Photocatalyst for Visible-Light-Driven Overall Water Splitting. J. Am. Chem. Soc. 2005, 127, 8286-8287. [CrossRef] [PubMed]

130. Maeda, K.; Teramura, K.; Domen, K. Effect of Post-Calcination on Photocatalytic Activity of $\left(\mathrm{Ga}_{1-x} \mathrm{Zn}_{x}\right)\left(\mathrm{N}_{1-x} \mathrm{O}_{x}\right)$ Solid Solution for Overall Water Splitting under Visible Light. J. Catal. 2008, 254, 198-204. [CrossRef]

131. Hisatomi, T.; Okamura, S.; Liu, J.; Shinohara, Y.; Ueda, K.; Higashi, T.; Katayama, M.; Minegishi, T.; Domen, K $\mathrm{La}_{5} \mathrm{Ti}_{2} \mathrm{Cu}_{1-x} \mathrm{Ag}_{x} \mathrm{~S}_{5} \mathrm{O}_{7}$ Photocathodes Operating at Positive Potentials during Photoelectrochemical Hydrogen Evolution under Irradiation of up to $710 \mathrm{~nm}$. Energy Environ. Sci. 2015, 8, 3354-3362. [CrossRef]

132. Nandy, S.; Goto, Y.; Hisatomi, T.; Moriya, Y.; Minegishi, T.; Katayama, M.; Domen, K. Synthesis and Photocatalytic Activity of $\mathrm{La}_{5} \mathrm{Ti}_{2} \mathrm{Cu}\left(\mathrm{S}_{1-x} \mathrm{Se}_{x}\right)_{5} \mathrm{O}_{7}$ Solid Solutions for $\mathrm{H}_{2}$ Production under Visible Light Irradiation. ChemPhotoChem 2017, 1, 265-272. [CrossRef]

133. Sahoo, P.P.; Maggard, P.A. Crystal Chemistry, Band Engineering, and Photocatalytic Activity of the $\mathrm{LiNb}_{3} \mathrm{O}_{8}-\mathrm{CuNb}_{3} \mathrm{O}_{8} \mathrm{Solid}$ Solution. Inorg. Chem. 2013, 52, 4443-4450. [CrossRef] [PubMed]

134. Liao, L.; Zhang, Q.; Su, Z.; Zhao, Z.; Wang, Y.; Li, Y.; Lu, X.; Wei, D.; Feng, G.; Yu, Q.; et al. Efficient Solar Water-Splitting Using a Nanocrystalline CoO Photocatalyst. Nat. Nanotechnol. 2014, 9, 69-73. [CrossRef]

135. Shi, W.; Guo, F.; Wang, H.; Guo, S.; Li, H.; Zhou, Y.; Zhu, C.; Liu, Y.; Huang, H.; Mao, B.; et al. New Insight of Water-Splitting Photocatalyst: $\mathrm{H}_{2} \mathrm{O}_{2}$-Resistance Poisoning and Photothermal Deactivation in Sub-Micrometer CoO Octahedrons. ACS Appl. Mater. Interfaces 2017, 9, 20585-20593. [CrossRef]

136. Galsin, J.S. Chapter 23-Defects in Crystalline Solids. In Solid State Physics; Galsin, J.S., Ed.; Academic Press: Cambridge, MA, USA, 2019; pp. 513-537.

137. Maeda, K.; Domen, K. Solid Solution of GaN and ZnO as a Stable Photocatalyst for Overall Water Splitting under Visible Light. Chem. Mater. 2010, 22, 612-623. [CrossRef] 
138. Wei, S.-H.; Zunger, A. Role of Metal $d$ States in II-VI Semiconductors. Phys. Rev. B 1988, 37, 8958-8981. [CrossRef]

139. Guo, F.; Shi, W.; Wang, H.; Han, M.; Li, H.; Huang, H.; Liu, Y.; Kang, Z. Facile Fabrication of a CoO/g-C $\mathrm{C}_{3} \mathrm{~N}_{4}$ p-n Heterojunction with Enhanced Photocatalytic Activity and Stability for Tetracycline Degradation under Visible Light. Catal. Sci. Technol. 2017, 7 , 3325-3331. [CrossRef]

140. Wang, S.; Zhao, L.; Huang, W.; Zhao, H.; Chen, J.; Cai, Q.; Jiang, X.; Lu, C.; Shi, W. Solvothermal Synthesis of CoO/BiVO 4 p-n Heterojunction with Micro-Nano Spherical Structure for Enhanced Visible Light Photocatalytic Activity towards Degradation of Tetracycline. Mater. Res. Bull. 2021, 135, 111161. [CrossRef]

141. Wang, Y.; Wu, Y.; Sun, K.; Mi, Z. A Quadruple-Band Metal-Nitride Nanowire Artificial Photosynthesis System for High Efficiency Photocatalytic Overall Solar Water Splitting. Mater. Horiz. 2019, 6, 1454-1462. [CrossRef]

142. She, X.; Liu, L.; Ji, H.; Mo, Z.; Li, Y.; Huang, L.; Du, D.; Xu, H.; Li, H. Template-Free Synthesis of 2D Porous Ultrathin NonmetalDoped g- $\mathrm{C}_{3} \mathrm{~N}_{4}$ Nanosheets with Highly Efficient Photocatalytic $\mathrm{H}_{2}$ Evolution from Water under Visible Light. Appl. Catal. B 2016, 187, 144-153. [CrossRef]

143. Fang, X.-X.; Ma, L.-B.; Liang, K.; Zhao, S.-J.; Jiang, Y.-F.; Ling, C.; Zhao, T.; Cheang, T.-Y.; Xu, A.-W. The Doping of Phosphorus Atoms into Graphitic Carbon Nitride for Highly Enhanced Photocatalytic Hydrogen Evolution. J. Mater. Chem. A 2019, 7, 11506-11512. [CrossRef]

144. Liu, G.; Niu, P.; Sun, C.; Smith, S.C.; Chen, Z.; Lu, G.Q.; Cheng, H.-M. Unique Electronic Structure Induced High Photoreactivity of Sulfur-Doped Graphitic $\mathrm{C}_{3} \mathrm{~N}_{4}$. J. Am. Chem. Soc. 2010, 132, 11642-11648. [CrossRef]

145. Hong, J.; Xia, X.; Wang, Y.; Xu, R. Mesoporous Carbon Nitride with in situ Sulfur Doping for Enhanced Photocatalytic Hydrogen Evolution from Water under Visible Light. J. Mater. Chem. 2012, 22, 15006-15012. [CrossRef]

146. Thaweesak, S.; Wang, S.; Lyu, M.; Xiao, M.; Peerakiatkhajohn, P.; Wang, L. Boron-Doped Graphitic Carbon Nitride Nanosheets for Enhanced Visible Light Photocatalytic Water Splitting. Dalton Trans. 2017, 46, 10714-10720. [CrossRef] [PubMed]

147. Wang, Y.; Di, Y.; Antonietti, M.; Li, H.; Chen, X.; Wang, X. Excellent Visible-Light Photocatalysis of Fluorinated Polymeric Carbon Nitride Solids. Chem. Mater. 2010, 22, 5119-5121. [CrossRef]

148. Han, Q.; Hu, C.; Zhao, F.; Zhang, Z.; Chen, N.; Qu, L. One-Step Preparation of Iodine-Doped Graphitic Carbon Nitride Nanosheets as Efficient Photocatalysts for Visible Light Water Splitting. J. Mater. Chem. A 2015, 3, 4612-4619. [CrossRef]

149. Gao, J.; Wang, Y.; Zhou, S.; Lin, W.; Kong, Y. A Facile One-Step Synthesis of Fe-Doped g- $\mathrm{C}_{3} \mathrm{~N}_{4}$ Nanosheets and Their Improved Visible-Light Photocatalytic Performance. ChemCatChem 2017, 9, 1708-1715. [CrossRef]

150. Le, S.; Jiang, T.; Zhao, Q.; Liu, X.; Li, Y.; Fang, B.; Gong, M. Cu-Doped Mesoporous Graphitic Carbon Nitride for Enhanced Visible-Light Driven Photocatalysis. RSC Adv. 2016, 6, 38811-38819. [CrossRef]

151. Yue, B.; Li, Q.; Iwai, H.; Kako, T.; Ye, J. Hydrogen Production Using Zinc-Doped Carbon Nitride Catalyst Irradiated with Visible Light. Sci. Technol. Adv. Mater. 2011, 12, 034401. [CrossRef]

152. Wu, C.; Xue, S.; Qin, Z.; Nazari, M.; Yang, G.; Yue, S.; Tong, T.; Ghasemi, H.; Hernandez, F.C.R.; Xue, S.; et al. Making g-C $3 \mathrm{~N}_{4}$ Ultra-Thin Nanosheets Active for Photocatalytic Overall Water Splitting. Appl. Catal. B 2021, 282, 119557. [CrossRef]

153. Zhang, F.-M.; Sheng, J.-L.; Yang, Z.-D.; Sun, X.-J.; Tang, H.-L.; Lu, M.; Dong, H.; Shen, F.-C.; Liu, J.; Lan, Y.-Q. Rational Design of MOF/COF Hybrid Materials for Photocatalytic $\mathrm{H}_{2}$ Evolution in the Presence of Sacrificial Electron Donors. Angew. Chem. Int. Ed. 2018, 57, 12106-12110. [CrossRef]

154. Chen, Y.; Yang, D.; Shi, B.; Dai, W.; Ren, H.; An, K.; Zhou, Z.; Zhao, Z.; Wang, W.; Jiang, Z. In situ Construction of HydrazoneLinked COF-Based Core-Shell Hetero-Frameworks for Enhanced Photocatalytic Hydrogen Evolution. J. Mater. Chem. A 2020, 8 , 7724-7732. [CrossRef]

155. Chowdhury, F.A.; Trudeau, M.L.; Guo, H.; Mi, Z. A Photochemical Diode Artificial Photosynthesis System for Unassisted High Efficiency Overall Pure Water Splitting. Nat. Commun. 2018, 9, 1707. [CrossRef] [PubMed]

156. Ong, W.-J.; Tan, L.-L.; Ng, Y.H.; Yong, S.-T.; Chai, S.-P. Graphitic Carbon Nitride (g- $\left.\mathrm{C}_{3} \mathrm{~N}_{4}\right)$-Based Photocatalysts for Artificial Photosynthesis and Environmental Remediation: Are We a Step Closer To Achieving Sustainability? Chem. Rev. 2016, 116, 7159-7329. [CrossRef]

157. Cao, S.; Low, J.; Yu, J.; Jaroniec, M. Polymeric Photocatalysts Based on Graphitic Carbon Nitride. Adv. Mater. 2015, 27, 2150-2176. [CrossRef]

158. Wen, J.; Xie, J.; Chen, X.; Li, X. A Review on g- $\mathrm{C}_{3} \mathrm{~N}_{4}$-Based Photocatalysts. Appl. Surf. Sci. 2017, 391, 72-123. [CrossRef]

159. Cheng, C.; Shi, J.; Wen, L.; Dong, C.-L.; Huang, Y.-C.; Zhang, Y.; Zong, S.; Diao, Z.; Shen, S.; Guo, L. Disordered Nitrogen-DefectRich Porous Carbon Nitride Photocatalyst for Highly Efficient $\mathrm{H}_{2}$ Evolution under Visible-Light Irradiation. Carbon 2021, 181, 193-203. [CrossRef]

160. Wang, X.; Maeda, K.; Thomas, A.; Takanabe, K.; Xin, G.; Carlsson, J.M.; Domen, K.; Antonietti, M. A Metal-Free Polymeric Photocatalyst for Hydrogen Production from Water under Visible Light. Nat. Mater. 2009, 8, 76-80. [CrossRef]

161. Rono, N.; Kibet, J.K.; Martincigh, B.S.; Nyamori, V.O. A Review of the Current Status of Graphitic Carbon Nitride. Crit. Rev. Solid State Mater. Sci. 2020, 46, 189-217. [CrossRef]

162. Wang, X.; Maeda, K.; Chen, X.; Takanabe, K.; Domen, K.; Hou, Y.; Fu, X.; Antonietti, M. Polymer Semiconductors for Artificial Photosynthesis: Hydrogen Evolution by Mesoporous Graphitic Carbon Nitride with Visible Light. J. Am. Chem. Soc. 2009, 131, 1680-1681. [CrossRef] [PubMed]

163. Xiang, Q.; Yu, J.; Jaroniec, M. Preparation and Enhanced Visible-Light Photocatalytic $\mathrm{H}_{2}$-Production Activity of Graphene/ $\mathrm{C}_{3} \mathrm{~N}_{4}$ Composites. J. Phys. Chem. C 2011, 115, 7355-7363. [CrossRef] 
164. Liu, J.; Liu, Y.; Liu, N.; Han, Y.; Zhang, X.; Huang, H.; Lifshitz, Y.; Lee, S.-T.; Zhong, J.; Kang, Z. Metal-Free Efficient Photocatalyst for Stable Visible Water Splitting via a Two-Electron Pathway. Science 2015, 347, 970-974. [CrossRef] [PubMed]

165. Fu, J.; Yu, J.; Jiang, C.; Cheng, B. g- $\mathrm{C}_{3} \mathrm{~N}_{4}$-Based Heterostructured Photocatalysts. Adv. Energy Mater. 2018, 8, 1701503. [CrossRef]

166. Fu, J.; Xu, Q.; Low, J.; Jiang, C.; Yu, J. Ultrathin 2D/2D WO $3 /$ g- $\mathrm{C}_{3} \mathrm{~N}_{4}$ Step-Scheme $\mathrm{H}_{2}$-Production Photocatalyst. Appl. Catal. B 2019, 243, 556-565. [CrossRef]

167. Gao, H.; Guo, Y.; Yu, Z.; Zhao, M.; Hou, Y.; Zhu, Z.; Yan, S.; Liu, Q.; Zou, Z. Incorporating $p$-Phenylene as an Electron-Donating Group into Graphitic Carbon Nitride for Efficient Charge Separation. ChemSusChem 2019, 12, 4285-4292. [CrossRef] [PubMed]

168. Zhang, L.; Ding, N.; Wu, J.; Iwasaki, K.; Lin, L.; Yamaguchi, Y.; Shibayama, Y.; Shi, J.; Wu, H.; Luo, Y.; et al. New Two-Dimensional Porous Graphitic Carbon Nitride Nanosheets for Highly Efficient Photocatalytic Hydrogen Evolution under Visible-Light Irradiation. Catal. Sci. Technol. 2018, 8, 3846-3852. [CrossRef]

169. Zhang, L.; Ding, N.; Hashimoto, M.; Iwasaki, K.; Chikamori, N.; Nakata, K.; Xu, Y.; Shi, J.; Wu, H.; Luo, Y.; et al. Sodium-Doped Carbon Nitride Nanotubes for Efficient Visible Light-Driven Hydrogen Production. Nano Res. 2018, 11, 2295-2309. [CrossRef]

170. Patnaik, S.; Sahoo, D.P.; Parida, K. Recent Advances in Anion Doped g- $\mathrm{C}_{3} \mathrm{~N}_{4}$ Photocatalysts: A Review. Carbon 2021, $172,682-711$. [CrossRef]

171. Gao, H.; Yan, S.; Wang, J.; Zou, Z. Ion Coordination Significantly Enhances the Photocatalytic Activity of Graphitic-Phase Carbon Nitride. Dalton Trans. 2014, 43, 8178-8183. [CrossRef]

172. Zhang, K.; Wang, L.; Sheng, X.; Ma, M.; Jung, M.S.; Kim, W.; Lee, H.; Park, J.H. Tunable Bandgap Energy and Promotion of $\mathrm{H}_{2} \mathrm{O}_{2}$ Oxidation for Overall Water Splitting from Carbon Nitride Nanowire Bundles. Adv. Energy Mater. 2016, 6, 1502352. [CrossRef]

173. Zeng, Y.; Li, H.; Luo, J.; Yuan, J.; Wang, L.; Liu, C.; Xia, Y.; Liu, M.; Luo, S.; Cai, T.; et al. Sea-Urchin-Structure g-C ${ }_{3} N_{4}$ with Narrow Bandgap $(\sim 2.0 \mathrm{eV})$ for Efficient Overall Water Splitting under Visible Light Irradiation. Appl. Catal. B 2019, $249,275-281$. [CrossRef]

174. Stegbauer, L.; Schwinghammer, K.; Lotsch, B.V. A Hydrazone-Based Covalent Organic Framework for Photocatalytic Hydrogen Production. Chem. Sci. 2014, 5, 2789-2793. [CrossRef]

175. Kandambeth, S.; Mallick, A.; Lukose, B.; Mane, M.V.; Heine, T.; Banerjee, R. Construction of Crystalline 2D Covalent Organic Frameworks with Remarkable Chemical (Acid/Base) Stability via a Combined Reversible and Irreversible Route. J. Am. Chem. Soc. 2012, 134, 19524-19527. [CrossRef] [PubMed]

176. Chen, X.; Addicoat, M.; Jin, E.; Zhai, L.; Xu, H.; Huang, N.; Guo, Z.; Liu, L.; Irle, S.; Jiang, D. Locking Covalent Organic Frameworks with Hydrogen Bonds: General and Remarkable Effects on Crystalline Structure, Physical Properties, and Photochemical Activity. J. Am. Chem. Soc. 2015, 137, 3241-3247. [CrossRef]

177. Chen, W.; Yang, Z.; Xie, Z.; Li, Y.; Yu, X.; Lu, F.; Chen, L. Benzothiadiazole Functionalized D-A Type Covalent Organic Frameworks for Effective Photocatalytic Reduction of Aqueous Chromium(VI). J. Mater. Chem. A 2019, 7, 998-1004. [CrossRef]

178. Nørskov, J.K.; Bligaard, T.; Logadottir, A.; Kitchin, J.R.; Chen, J.G.; Pandelov, S.; Stimming, U. Trends in the Exchange Current for Hydrogen Evolution. J. Electrochem. Soc. 2005, 152, J23-J26. [CrossRef]

179. Jaramillo Thomas, F.; Jørgensen Kristina, P.; Bonde, J.; Nielsen Jane, H.; Horch, S.; Chorkendorff, I. Identification of Active Edge Sites for Electrochemical $\mathrm{H}_{2}$ Evolution from $\mathrm{MoS}_{2}$ Nanocatalysts. Science 2007, 317, 100-102. [CrossRef]

180. Zheng, Y.; Jiao, Y.; Zhu, Y.; Li, L.H.; Han, Y.; Chen, Y.; Du, A.; Jaroniec, M.; Qiao, S.Z. Hydrogen Evolution by a Metal-Free Electrocatalyst. Nat. Commun. 2014, 5, 3783. [CrossRef]

181. Duonghong, D.; Borgarello, E.; Grätzel, M. Dynamics of Light-Induced Water Cleavage in Colloidal Systems. J. Am. Chem. Soc. 1981, 103, 4685-4690. [CrossRef]

182. Maeda, K. Direct Splitting of Pure Water into Hydrogen and Oxygen Using Rutile Titania Powder as a Photocatalyst. Chem. Commun. 2013, 49, 8404-8406. [CrossRef]

183. Oshima, T.; Lu, D.; Ishitani, O.; Maeda, K. Intercalation of Highly Dispersed Metal Nanoclusters into a Layered Metal Oxide for Photocatalytic Overall Water Splitting. Angew. Chem. Int. Ed. 2015, 54, 2698-2702. [CrossRef] [PubMed]

184. Ahmad, M.; Quan, X.; Chen, S.; Yu, H.; Zhenxing, Z. Operating Redox Couple Transport Mechanism for Enhancing Photocatalytic $\mathrm{H}_{2}$ Generation of $\mathrm{Pt}$ and $\mathrm{CrO}_{x}$-Decorated ZnCdS Nanocrystals. Appl. Catal. B 2021, 283, 119601. [CrossRef]

185. Yamaguti, K.; Sato, S. Photolysis of Water over Metallized Powdered Titanium Dioxide. J. Chem. Soc. Faraday Trans. 1 1985, 81, 1237-1246. [CrossRef]

186. Zong, X.; Yan, H.; Wu, G.; Ma, G.; Wen, F.; Wang, L.; Li, C. Enhancement of Photocatalytic $\mathrm{H}_{2}$ Evolution on CdS by Loading MoS 2 as Cocatalyst under Visible Light Irradiation. J. Am. Chem. Soc. 2008, 130, 7176-7177. [CrossRef]

187. Zong, X.; Wu, G.; Yan, H.; Ma, G.; Shi, J.; Wen, F.; Wang, L.; Li, C. Photocatalytic $\mathrm{H}_{2}$ Evolution on MoS $2 /$ CdS Catalysts under Visible Light Irradiation. J. Phys. Chem. C 2010, 114, 1963-1968. [CrossRef]

188. Zhu, B.; Lin, B.; Zhou, Y.; Sun, P.; Yao, Q.; Chen, Y.; Gao, B. Enhanced Photocatalytic $\mathrm{H}_{2}$ Evolution on ZnS Loaded with Graphene and $\mathrm{MoS}_{2}$ Nanosheets as Cocatalysts. J. Mater. Chem. A 2014, 2, 3819-3827. [CrossRef]

189. Sun, B.; Zhou, W.; Li, H.; Ren, L.; Qiao, P.; Li, W.; Fu, H. Synthesis of Particulate Hierarchical Tandem Heterojunctions toward Optimized Photocatalytic Hydrogen Production. Adv. Mater. 2018, 30, 1804282. [CrossRef]

190. Tabata, M.; Maeda, K.; Ishihara, T.; Minegishi, T.; Takata, T.; Domen, K. Photocatalytic Hydrogen Evolution from Water Using Copper Gallium Sulfide under Visible-Light Irradiation. J. Phys. Chem. C 2010, 114, 11215-11220. [CrossRef]

191. Hong, J.; Wang, Y.; Wang, Y.; Zhang, W.; Xu, R. Noble-Metal-Free NiS/ $\mathrm{C}_{3} \mathrm{~N}_{4}$ for Efficient Photocatalytic Hydrogen Evolution from Water. ChemSusChem 2013, 6, 2263-2268. [CrossRef] 
192. Zhao, H.; Zhang, H.; Cui, G.; Dong, Y.; Wang, G.; Jiang, P.; Wu, X.; Zhao, N. A Photochemical Synthesis Route to Typical Transition Metal Sulfides as Highly Efficient Cocatalyst for Hydrogen Evolution: From the Case of NiS/g-C $3 \mathrm{~N}_{4}$. Appl. Catal. B 2018, 225, 284-290. [CrossRef]

193. Li, N.; Huang, H.; Bibi, R.; Shen, Q.; Ngulube, R.; Zhou, J.; Liu, M. Noble-Metal-Free MOF Derived Hollow $\mathrm{CdS}_{\text {TiO }} 2$ Decorated with NiS Cocatalyst for Efficient Photocatalytic Hydrogen Evolution. Appl. Surf. Sci. 2019, 476, 378-386. [CrossRef]

194. Chen, Z.; Cheng, C.; Xing, F.; Huang, C. Strong Interfacial Coupling for NiS Thin Layer Covered CdS Nanorods with Highly Efficient Photocatalytic Hydrogen Production. New J. Chem. 2020, 44, 19083-19090. [CrossRef]

195. Wang, M.; Cheng, J.; Wang, X.; Hong, X.; Fan, J.; Yu, H. Sulfur-Mediated Photodeposition Synthesis of NiS Cocatalyst for Boosting $\mathrm{H}_{2}$-Evolution Performance of g- $\mathrm{C}_{3} \mathrm{~N}_{4}$ Photocatalyst. Chin. J. Catal. 2021, 42, 37-45. [CrossRef]

196. Lei, Y.; Hou, J.; Wang, F.; Ma, X.; Jin, Z.; Xu, J.; Min, S. Boosting the Catalytic Performance of MoS ${ }_{x}$ Cocatalysts over CdS Nanoparticles for Photocatalytic $\mathrm{H}_{2}$ Evolution by Co Doping via a Facile Photochemical Route. Appl. Surf. Sci. 2017, 420, 456-464. [CrossRef]

197. Liu, W.; Wang, X.; Yu, H.; Yu, J. Direct Photoinduced Synthesis of Amorphous CoMoS ${ }_{x}$ Cocatalyst and Its Improved Photocatalytic $\mathrm{H}_{2}$-Evolution Activity of CdS. ACS Sustain. Chem. Eng. 2018, 6, 12436-12445. [CrossRef]

198. Xue, Y.; Min, S.; Meng, J.; Liu, X.; Lei, Y.; Tian, L.; Wang, F. Light-Induced Confined Growth of Amorphous Co Doped MoS $x$ Nanodots on $\mathrm{TiO}_{2}$ Nanoparticles for Efficient and Stable in situ Photocatalytic $\mathrm{H}_{2}$ Evolution. Int. J. Hydrogen Energy 2019, 44, 8133-8143. [CrossRef]

199. Lei, Y.; Zhang, Y.; Li, Z.; Xu, S.; Huang, J.; Ng, K.H.; Lai, Y. Molybdenum Sulfide Cocatalyst Activation upon Photodeposition of Cobalt for Improved Photocatalytic Hydrogen Production Activity of ZnCdS. Chem. Eng. J. 2021, 425, 131478. [CrossRef]

200. Sun, Z.; Zheng, H.; Li, J.; Du, P. Extraordinarily Efficient Photocatalytic Hydrogen Evolution in Water Using Semiconductor Nanorods Integrated with Crystalline $\mathrm{Ni}_{2} \mathrm{P}$ Cocatalysts. Energy Environ. Sci. 2015, 8, 2668-2676. [CrossRef]

201. Chen, Y.; Qin, Z. General Applicability of Nanocrystalline $\mathrm{Ni}_{2} \mathrm{P}$ as a Noble-Metal-Free Cocatalyst to Boost Photocatalytic Hydrogen Generation. Catal. Sci. Technol. 2016, 6, 8212-8221. [CrossRef]

202. Indra, A.; Acharjya, A.; Menezes, P.W.; Merschjann, C.; Hollmann, D.; Schwarze, M.; Aktas, M.; Friedrich, A.; Lochbrunner, S.; Thomas, A.; et al. Boosting Visible-Light-Driven Photocatalytic Hydrogen Evolution with an Integrated Nickel Phosphide-Carbon Nitride System. Angew. Chem. Int. Ed. 2017, 56, 1653-1657. [CrossRef] [PubMed]

203. Ye, P.; Liu, X.; Iocozzia, J.; Yuan, Y.; Gu, L.; Xu, G.; Lin, Z. A Highly Stable Non-Noble Metal Ni ${ }_{2} \mathrm{P}$ Co-Catalyst for Increased $\mathrm{H}_{2}$ Generation by $\mathrm{g}-\mathrm{C}_{3} \mathrm{~N}_{4}$ under Visible Light Irradiation. J. Mater. Chem. A 2017, 5, 8493-8498. [CrossRef]

204. Liu, E.; Jin, C.; Xu, C.; Fan, J.; Hu, X. Facile Strategy to Fabricate $\mathrm{Ni}_{2} \mathrm{P} / \mathrm{g}-\mathrm{C}_{3} \mathrm{~N}_{4}$ Heterojunction with Excellent Photocatalytic Hydrogen Evolution Activity. Int. J. Hydrogen Energy 2018, 43, 21355-21364. [CrossRef]

205. Cheng, H.; Lv, X.-J.; Cao, S.; Zhao, Z.-Y.; Chen, Y.; Fu, W.-F. Robustly Photogenerating $\mathrm{H}_{2}$ in Water Using FeP/CdS Catalyst under Solar Irradiation. Sci. Rep. 2016, 6, 19846. [CrossRef]

206. Zhao, H.; Wang, J.; Dong, Y.; Jiang, P. Noble-Metal-Free Iron Phosphide Cocatalyst Loaded Graphitic Carbon Nitride as an Efficient and Robust Photocatalyst for Hydrogen Evolution under Visible Light Irradiation. ACS Sustain. Chem. Eng. 2017, 5 , 8053-8060. [CrossRef]

207. Zeng, D.; Zhou, T.; Ong, W.-J.; Wu, M.; Duan, X.; Xu, W.; Chen, Y.; Zhu, Y.-A.; Peng, D.-L. Sub-5 nm Ultra-Fine FeP Nanodots as Efficient Co-Catalysts Modified Porous $\mathrm{g}_{-} \mathrm{C}_{3} \mathrm{~N}_{4}$ for Precious-Metal-Free Photocatalytic Hydrogen Evolution under Visible Light. ACS Appl. Mater. Interfaces 2019, 11, 5651-5660. [CrossRef] [PubMed]

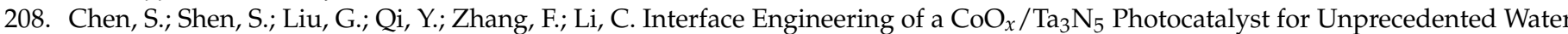
Oxidation Performance under Visible-Light-Irradiation. Angew. Chem. Int. Ed. 2015, 54, 3047-3051. [CrossRef] [PubMed]

209. Zhang, J.; Yu, Z.; Gao, Z.; Ge, H.; Zhao, S.; Chen, C.; Chen, S.; Tong, X.; Wang, M.; Zheng, Z.; et al. Porous TiO 2 Nanotubes with Spatially Separated Platinum and $\mathrm{CoO}_{x}$ Cocatalysts Produced by Atomic Layer Deposition for Photocatalytic Hydrogen Production. Angew. Chem. Int. Ed. 2017, 56, 816-820. [CrossRef]

210. Zhang, G.; Lan, Z.-A.; Lin, L.; Lin, S.; Wang, X. Overall Water Splitting by Pt/g- $\mathrm{C}_{3} \mathrm{~N}_{4}$ Photocatalysts without Using Sacrificial Agents. Chem. Sci. 2016, 7, 3062-3066. [CrossRef] [PubMed]

211. Zhang, W.; Tang, J.; Ye, J. Structural, Photocatalytic, and Photophysical Properties of Perovskite $\mathrm{MSnO} 3(\mathrm{M}=\mathrm{Ca}, \mathrm{Sr}$, and Ba) Photocatalysts. J. Mater. Res. 2007, 22, 1859-1871. [CrossRef]

212. Maeda, K.; Wang, X.; Nishihara, Y.; Lu, D.; Antonietti, M.; Domen, K. Photocatalytic Activities of Graphitic Carbon Nitride Powder for Water Reduction and Oxidation under Visible Light. J. Phys. Chem. C 2009, 113, 4940-4947. [CrossRef]

213. Maeda, K.; Abe, R.; Domen, K. Role and Function of Ruthenium Species as Promoters with TaON-Based Photocatalysts for Oxygen Evolution in Two-Step Water Splitting under Visible Light. J. Phys. Chem. C 2011, 115, 3057-3064. [CrossRef]

214. Kamat, P.V.; Bisquert, J. Solar Fuels. Photocatalytic Hydrogen Generation. J. Phys. Chem. C 2013, 117, 14873-14875. [CrossRef]

215. Yan, H.; Yang, J.; Ma, G.; Wu, G.; Zong, X.; Lei, Z.; Shi, J.; Li, C. Visible-Light-Driven Hydrogen Production with Extremely High Quantum Efficiency on Pt-PdS/CdS Photocatalyst. J. Catal. 2009, 266, 165-168. [CrossRef]

216. Li, X.-L.; Yang, G.-Q.; Li, S.-S.; Xiao, N.; Li, N.; Gao, Y.-Q.; Lv, D.; Ge, L. Novel Dual Co-Catalysts Decorated Au@HCS@PdS Hybrids with Spatially Separated Charge Carriers and Enhanced Photocatalytic Hydrogen Evolution Activity. Chem. Eng. J. 2020, 379, 122350. [CrossRef]

217. Man, I.C.; Su, H.-Y.; Calle-Vallejo, F.; Hansen, H.A.; Martínez, J.I.; Inoglu, N.G.; Kitchin, J.; Jaramillo, T.F.; Nørskov, J.K.; Rossmeisl, J. Universality in Oxygen Evolution Electrocatalysis on Oxide Surfaces. ChemCatChem 2011, 3, 1159-1165. [CrossRef] 
218. Ikeda, T.; Xiong, A.; Yoshinaga, T.; Maeda, K.; Domen, K.; Teranishi, T. Polyol Synthesis of Size-Controlled Rh Nanoparticles and Their Application to Photocatalytic Overall Water Splitting under Visible Light. J. Phys. Chem. C 2013, 117, 2467-2473. [CrossRef]

219. Qin, L.; Si, G.; Li, X.; Kang, S.-Z. Synergetic Effect of Cu-Pt Bimetallic Cocatalyst on $\mathrm{SrTiO}_{3}$ for Efficient Photocatalytic Hydrogen Production from Water. RSC Adv. 2015, 5, 102593-102598. [CrossRef]

220. Han, C.; Lu, Y.; Zhang, J.; Ge, L.; Li, Y.; Chen, C.; Xin, Y.; Wu, L.; Fang, S. Novel PtCo Alloy Nanoparticle Decorated 2D g-C ${ }_{3} N_{4}$ Nanosheets with Enhanced Photocatalytic Activity for $\mathrm{H}_{2}$ Evolution under Visible Light Irradiation. J. Mater. Chem. A 2015, 3, 23274-23282. [CrossRef]

221. Cao, S.; Jiang, J.; Zhu, B.; Yu, J. Shape-Dependent Photocatalytic Hydrogen Evolution Activity over a Pt Nanoparticle Coupled g- $\mathrm{C}_{3} \mathrm{~N}_{4}$ Photocatalyst. Phys. Chem. Chem. Phys. 2016, 18, 19457-19463. [CrossRef]

222. Cao, S.; Li, H.; Li, Y.; Zhu, B.; Yu, J. Dependence of Exposed Facet of Pd on Photocatalytic $\mathrm{H}_{2}$-Production Activity. ACS Sustain. Chem. Eng. 2018, 6, 6478-6487. [CrossRef]

223. Luo, M.; Yao, W.; Huang, C.; Wu, Q.; Xu, Q. Shape-Controlled Synthesis of Pd Nanoparticles for Effective Photocatalytic Hydrogen Production. RSC Adv. 2015, 5, 40892-40898. [CrossRef]

224. Luo, M.; Lu, P.; Yao, W.; Huang, C.; Xu, Q.; Wu, Q.; Kuwahara, Y.; Yamashita, H. Shape and Composition Effects on Photocatalytic Hydrogen Production for Pt-Pd Alloy Cocatalysts. ACS Appl. Mater. Interfaces 2016, 8, 20667-20674. [CrossRef]

225. Wang, Z.; Luo, Y.; Hisatomi, T.; Vequizo, J.J.M.; Suzuki, S.; Chen, S.; Nakabayashi, M.; Lin, L.; Pan, Z.; Kariya, N.; et al. Sequential Cocatalyst Decoration on $\mathrm{BaTaO}_{2} \mathrm{~N}$ towards Highly-Active Z-Scheme Water Splitting. Nat. Commun. 2021, 12, 1005. [CrossRef]

226. Maeda, K.; Teramura, K.; Lu, D.; Saito, N.; Inoue, Y.; Domen, K. Noble-Metal/ $\mathrm{Cr}_{2} \mathrm{O}_{3}$ Core/Shell Nanoparticles as a Cocatalyst for Photocatalytic Overall Water Splitting. Angew. Chem. Int. Ed. 2006, 45, 7806-7809. [CrossRef] [PubMed]

227. Maeda, K.; Teramura, K.; Lu, D.; Saito, N.; Inoue, Y.; Domen, K. Roles of Rh/ $\mathrm{Cr}_{2} \mathrm{O}_{3}$ (Core/Shell) Nanoparticles Photodeposited on Visible-Light-Responsive $\left(\mathrm{Ga}_{1-x} \mathrm{Zn}_{x}\right)\left(\mathrm{N}_{1-x} \mathrm{O}_{x}\right)$ Solid Solutions in Photocatalytic Overall Water Splitting. J. Phys. Chem. C 2007, 111, 7554-7560. [CrossRef]

228. Maeda, K.; Sakamoto, N.; Ikeda, T.; Ohtsuka, H.; Xiong, A.; Lu, D.; Kanehara, M.; Teranishi, T.; Domen, K. Preparation of Core-Shell-Structured Nanoparticles (with a Noble-Metal or Metal Oxide Core and a Chromia Shell) and Their Application in Water Splitting by Means of Visible Light. Chem. Eur. J. 2010, 16, 7750-7759. [CrossRef]

229. Xiong, A.; Yoshinaga, T.; Ikeda, T.; Takashima, M.; Hisatomi, T.; Maeda, K.; Setoyama, T.; Teranishi, T.; Domen, K. Effect of Hydrogen and Oxygen Evolution Cocatalysts on Photocatalytic Activity of GaN:ZnO. Eur. J. Inorg. Chem. 2014, $2014,767-772$. [CrossRef]

230. Wang, Z.; Inoue, Y.; Hisatomi, T.; Ishikawa, R.; Wang, Q.; Takata, T.; Chen, S.; Shibata, N.; Ikuhara, Y.; Domen, K. Overall Water Splitting by $\mathrm{Ta}_{3} \mathrm{~N}_{5}$ Nanorod Single Crystals Grown on the Edges of $\mathrm{KTaO}_{3}$ Particles. Nat. Catal. 2018, 1, 756-763. [CrossRef]

231. Takata, T.; Pan, C.; Nakabayashi, M.; Shibata, N.; Domen, K. Fabrication of a Core-Shell-Type Photocatalyst via Photodeposition of Group IV and V Transition Metal Oxyhydroxides: An Effective Surface Modification Method for Overall Water Splitting. J. Am. Chem. Soc. 2015, 137, 9627-9634. [CrossRef] [PubMed]

232. Yoshida, M.; Takanabe, K.; Maeda, K.; Ishikawa, A.; Kubota, J.; Sakata, Y.; Ikezawa, Y.; Domen, K. Role and Function of NobleMetal/Cr-Layer Core/Shell Structure Cocatalysts for Photocatalytic Overall Water Splitting Studied by Model Electrodes. J. Phys. Chem. C 2009, 113, 10151-10157. [CrossRef]

233. Ravi, P.; Rao, V.N.; Shankar, M.V.; Sathish, M. CuO- $\mathrm{Cr}_{2} \mathrm{O}_{3}$ Core-Shell Structured Co-Catalysts on TiO 2 for Efficient Photocatalytic Water Splitting Using Direct Solar Light. Int. J. Hydrogen Energy 2018, 43, 3976-3987. [CrossRef]

234. Pan, C.; Takata, T.; Nakabayashi, M.; Matsumoto, T.; Shibata, N.; Ikuhara, Y.; Domen, K. A Complex Perovskite-Type Oxynitride: The First Photocatalyst for Water Splitting Operable at up to $600 \mathrm{~nm}$. Angew. Chem. Int. Ed. 2015, 54, 2955-2959. [CrossRef] [PubMed]

235. Pan, C.; Takata, T.; Domen, K. Overall Water Splitting on the Transition-Metal Oxynitride Photocatalyst $\mathrm{LaMg}_{1 / 3} \mathrm{Ta}_{2 / 3} \mathrm{O}_{2} \mathrm{~N}$ over a Large Portion of the Visible-Light Spectrum. Chem. Eur. J. 2016, 22, 1854-1862. [CrossRef]

236. Maeda, K.; Teramura, K.; Lu, D.; Takata, T.; Saito, N.; Inoue, Y.; Domen, K. Photocatalyst Releasing Hydrogen from Water. Nature 2006, 440, 295. [CrossRef]

237. Maeda, K.; Teramura, K.; Masuda, H.; Takata, T.; Saito, N.; Inoue, Y.; Domen, K. Efficient Overall Water Splitting under VisibleLight Irradiation on $\left(\mathrm{Ga}_{1-x} \mathrm{Zn}_{x}\right)\left(\mathrm{N}_{1-x} \mathrm{O}_{x}\right)$ Dispersed with $\mathrm{Rh}-\mathrm{Cr}$ Mixed-Oxide Nanoparticles: Effect of Reaction Conditions on Photocatalytic Activity. J. Phys. Chem. B 2006, 110, 13107-13112. [CrossRef]

238. Maeda, K.; Teramura, K.; Lu, D.; Takata, T.; Saito, N.; Inoue, Y.; Domen, K. Characterization of Rh-Cr Mixed-Oxide Nanoparticles Dispersed on $\left(\mathrm{Ga}_{1-x} \mathrm{Zn}_{x}\right)\left(\mathrm{N}_{1-x} \mathrm{O}_{x}\right)$ as a Cocatalyst for Visible-Light-Driven Overall Water Splitting. J. Phys. Chem. B 2006, 110, 13753-13758. [CrossRef]

239. Hisatomi, T.; Maeda, K.; Takanabe, K.; Kubota, J.; Domen, K. Aspects of the Water Splitting Mechanism on $\left(\mathrm{Ga}_{1-x} \mathrm{Zn}_{x}\right)\left(\mathrm{N}_{1-x} \mathrm{O}_{x}\right)$ Photocatalyst Modified with $\mathrm{Rh}_{2-y} \mathrm{Cr}_{y} \mathrm{O}_{3}$ Cocatalyst. J. Phys. Chem. C 2009, 113, 21458-21466. [CrossRef]

240. Chiang, T.H.; Lyu, H.; Hisatomi, T.; Goto, Y.; Takata, T.; Katayama, M.; Minegishi, T.; Domen, K. Efficient Photocatalytic Water Splitting Using Al-Doped $\mathrm{SrTiO}_{3}$ Coloaded with Molybdenum Oxide and Rhodium-Chromium Oxide. ACS Catal. 2018, 8, 2782-2788. [CrossRef]

241. Nishino, T.; Saruyama, M.; Li, Z.; Nagatsuma, Y.; Nakabayashi, M.; Shibata, N.; Yamada, T.; Takahata, R.; Yamazoe, S.; Hisatomi, T.; et al. Self-Activated Rh-Zr Mixed Oxide as a Nonhazardous Cocatalyst for Photocatalytic Hydrogen Evolution. Chem. Sci. 2020, 11, 6862-6867. [CrossRef] 
242. Kanazawa, T.; Kato, K.; Yamaguchi, R.; Uchiyama, T.; Lu, D.; Nozawa, S.; Yamakata, A.; Uchimoto, Y.; Maeda, K. Cobalt Aluminate Spinel as a Cocatalyst for Photocatalytic Oxidation of Water: Significant Hole-Trapping Effect. ACS Catal. 2020, 10, 4960-4966. [CrossRef]

243. Xiao, S.Y.; Liu, Y.; Wu, X.F.; Gan, L.T.; Lin, H.Y.; Zheng, L.R.; Dai, S.; Liu, P.F.; Yang, H.G. A Low-Valent Cobalt Oxide Co-Catalyst to Boost Photocatalytic Water Oxidation via Enhanced Hole-Capturing Ability. J. Mater. Chem. A 2021, 9, 14786-14792. [CrossRef]

244. Negishi, Y.; Kurashige, W.; Niihori, Y.; Iwasa, T.; Nobusada, K. Isolation, Structure, and Stability of a Dodecanethiolate-Protected $\mathrm{Pd}_{1} \mathrm{Au}_{24}$ Cluster. Phys. Chem. Chem. Phys. 2010, 12, 6219-6225. [CrossRef]

245. Negishi, Y.; Kurashige, W.; Kobayashi, Y.; Yamazoe, S.; Kojima, N.; Seto, M.; Tsukuda, T. Formation of a Pd@Au 12 Superatomic Core in $\mathrm{Au}_{24} \mathrm{Pd}_{1}\left(\mathrm{SC}_{12} \mathrm{H}_{25}\right)_{18}$ Probed by ${ }^{197} \mathrm{Au}$ Mössbauer and Pd K-Edge EXAFS Spectroscopy. J. Phys. Chem. Lett. 2013, 4, 3579-3583. [CrossRef]

246. Negishi, Y.; Munakata, K.; Ohgake, W.; Nobusada, K. Effect of Copper Doping on Electronic Structure, Geometric Structure, and Stability of Thiolate-Protected $\mathrm{Au}_{25}$ Nanoclusters. J. Phys. Chem. Lett. 2012, 3, 2209-2214. [CrossRef] [PubMed]

247. Niihori, Y.; Matsuzaki, M.; Pradeep, T.; Negishi, Y. Separation of Precise Compositions of Noble Metal Clusters Protected with Mixed Ligands. J. Am. Chem. Soc. 2013, 135, 4946-4949. [CrossRef] [PubMed]

248. Niihori, Y.; Matsuzaki, M.; Uchida, C.; Negishi, Y. Advanced Use of High-Performance Liquid Chromatography for Synthesis of Controlled Metal Clusters. Nanoscale 2014, 6, 7889-7896. [CrossRef]

249. Niihori, Y.; Kikuchi, Y.; Kato, A.; Matsuzaki, M.; Negishi, Y. Understanding Ligand-Exchange Reactions on Thiolate-Protected Gold Clusters by Probing Isomer Distributions Using Reversed-Phase High-Performance Liquid Chromatography. ACS Nano 2015, 9, 9347-9356. [CrossRef] [PubMed]

250. Niihori, Y.; Eguro, M.; Kato, A.; Sharma, S.; Kumar, B.; Kurashige, W.; Nobusada, K.; Negishi, Y. Improvements in the LigandExchange Reactivity of Phenylethanethiolate-Protected $\mathrm{Au}_{25}$ Nanocluster by Ag or Cu Incorporation. J. Phys. Chem. C 2016, 120, 14301-14309. [CrossRef]

251. Niihori, Y.; Koyama, Y.; Watanabe, S.; Hashimoto, S.; Hossain, S.; Nair, L.V.; Kumar, B.; Kurashige, W.; Negishi, Y. Atomic and Isomeric Separation of Thiolate-Protected Alloy Clusters. J. Phys. Chem. Lett. 2018, 9, 4930-4934. [CrossRef]

252. Niihori, Y.; Hashimoto, S.; Koyama, Y.; Hossain, S.; Kurashige, W.; Negishi, Y. Dynamic Behavior of Thiolate-Protected Gold-Silver 38-Atom Alloy Clusters in Solution. J. Phys. Chem. C 2019, 123, 13324-13329. [CrossRef]

253. Niihori, Y.; Shima, D.; Yoshida, K.; Hamada, K.; Nair, L.V.; Hossain, S.; Kurashige, W.; Negishi, Y. High-Performance Liquid Chromatography Mass Spectrometry of Gold and Alloy Clusters Protected by Hydrophilic Thiolates. Nanoscale 2018, 10, 1641-1649. [CrossRef] [PubMed]

254. Negishi, Y.; Iwai, T.; Ide, M. Continuous Modulation of Electronic Structure of Stable Thiolate-Protected Au ${ }_{25}$ Cluster by Ag Doping. Chem. Commun. 2010, 46, 4713-4715. [CrossRef]

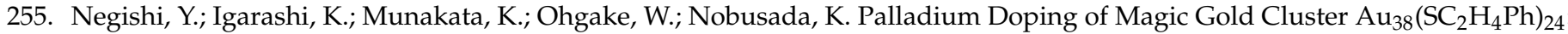
Formation of $\mathrm{Pd}_{2} \mathrm{Au}_{36}\left(\mathrm{SC}_{2} \mathrm{H}_{4} \mathrm{Ph}\right)_{24}$ with Higher Stability than $\mathrm{Au}_{38}\left(\mathrm{SC}_{2} \mathrm{H}_{4} \mathrm{Ph}\right)_{24}$. Chem. Commun. 2012, 48, 660-662. [CrossRef]

256. Negishi, Y.; Kurashige, W.; Niihori, Y.; Nobusada, K. Toward the Creation of Stable, Functionalized Metal Clusters. Phys. Chem. Chem. Phys. 2013, 15, 18736-18751. [CrossRef] [PubMed]

257. Kurashige, W.; Munakata, K.; Nobusada, K.; Negishi, Y. Synthesis of Stable $\mathrm{Cu}_{n} \mathrm{Au}_{25-n}$ Nanoclusters $(n=1-9)$ Using Selenolate Ligands. Chem. Commun. 2013, 49, 5447-5449. [CrossRef]

258. Niihori, Y.; Kurashige, W.; Matsuzaki, M.; Negishi, Y. Remarkable Enhancement in Ligand-Exchange Reactivity of ThiolateProtected $\mathrm{Au}_{25}$ Nanoclusters by Single Pd Atom Doping. Nanoscale 2013, 5, 508-512. [CrossRef]

259. Puls, A.; Jerabek, P.; Kurashige, W.; Förster, M.; Molon, M.; Bollermann, T.; Winter, M.; Gemel, C.; Negishi, Y.; Frenking, G.; et al. A Novel Concept for the Synthesis of Multiply Doped Gold Clusters [(M@ $\left.\left.\mathrm{Au}_{n} \mathrm{M}_{m}^{\prime}\right) \mathrm{L}_{k}\right]^{q+}$. Angew. Chem. Int. Ed. 2014, 53, 4327-4331. [CrossRef] [PubMed]

260. Negishi, Y. Toward the Creation of Functionalized Metal Nanoclusters and Highly Active Photocatalytic Materials Using Thiolate-Protected Magic Gold Clusters. Bull. Chem. Soc. Jpn. 2014, 87, 375-389. [CrossRef]

261. Kurashige, W.; Niihori, Y.; Sharma, S.; Negishi, Y. Recent Progress in the Functionalization Methods of Thiolate-Protected Gold Clusters. J. Phys. Chem. Lett. 2014, 5, 4134-4142. [CrossRef]

262. Sharma, S.; Kurashige, W.; Nobusada, K.; Negishi, Y. Effect of Trimetallization in Thiolate-Protected $\mathrm{Au}_{24-} \mathrm{Cu}_{n} \mathrm{Pd} \mathrm{Clusters}$ Nanoscale 2015, 7, 10606-10612. [CrossRef]

263. Sharma, S.; Yamazoe, S.; Ono, T.; Kurashige, W.; Niihori, Y.; Nobusada, K.; Tsukuda, T.; Negishi, Y. Tuning the Electronic Structure of Thiolate-Protected 25-Atom Clusters by Co-Substitution with Metals Having Different Preferential Sites. Dalton Trans. 2016, 45 18064-18068. [CrossRef]

264. Niihori, Y.; Uchida, C.; Kurashige, W.; Negishi, Y. High-Resolution Separation of Thiolate-Protected Gold Clusters by ReversedPhase High-Performance Liquid Chromatography. Phys. Chem. Chem. Phys. 2016, 18, 4251-4265. [CrossRef]

265. Kurashige, W.; Niihori, Y.; Sharma, S.; Negishi, Y. Precise Synthesis, Functionalization and Application of Thiolate-Protected Gold Clusters. Coord. Chem. Rev. 2016, 320-321, 238-250. [CrossRef]

266. Niihori, Y.; Hossain, S.; Kumar, B.; Nair, L.V.; Kurashige, W.; Negishi, Y. Perspective: Exchange Reactions in Thiolate-Protected Metal Clusters. APL Mater. 2017, 5, 053201. [CrossRef] 
267. Niihori, Y.; Hossain, S.; Sharma, S.; Kumar, B.; Kurashige, W.; Negishi, Y. Understanding and Practical Use of Ligand and Metal Exchange Reactions in Thiolate-Protected Metal Clusters to Synthesize Controlled Metal Clusters. Chem. Rec. 2017, 17, 473-484. [CrossRef] [PubMed]

268. Hossain, S.; Ono, T.; Yoshioka, M.; Hu, G.; Hosoi, M.; Chen, Z.; Nair, L.V.; Niihori, Y.; Kurashige, W.; Jiang, D.-e.; et al. ThiolateProtected Trimetallic $\mathrm{Au}_{\sim 20} \mathrm{Ag}_{\sim 4} \mathrm{Pd}$ and $\mathrm{Au}_{\sim 20} \mathrm{Ag}_{\sim 4} \mathrm{Pt}$ Alloy Clusters with Controlled Chemical Composition and Metal Positions. J. Phys. Chem. Lett. 2018, 9, 2590-2594. [CrossRef]

269. Nair, L.V.; Hossain, S.; Takagi, S.; Imai, Y.; Hu, G.; Wakayama, S.; Kumar, B.; Kurashige, W.; Jiang, D.-E.; Negishi, Y. HeteroBiicosahedral $\left[\mathrm{Au}_{24} \mathrm{Pd}\left(\mathrm{PPh}_{3}\right)_{10}\left(\mathrm{SC}_{2} \mathrm{H}_{4} \mathrm{Ph}\right)_{5} \mathrm{Cl}_{2}\right]^{+}$Nanocluster: Selective Synthesis and Optical and Electrochemical Properties. Nanoscale 2018, 10, 18969-18979. [CrossRef] [PubMed]

270. Hossain, S.; Niihori, Y.; Nair, L.V.; Kumar, B.; Kurashige, W.; Negishi, Y. Alloy Clusters: Precise Synthesis and Mixing Effects. Acc. Chem. Res. 2018, 51, 3114-3124. [CrossRef]

271. Niihori, Y.; Yoshida, K.; Hossain, S.; Kurashige, W.; Negishi, Y. Deepening the Understanding of Thiolate-Protected Metal Clusters Using High-Performance Liquid Chromatography. Bull. Chem. Soc. Jpn. 2019, 92, 664-695. [CrossRef]

272. Hossain, S.; Imai, Y.; Suzuki, D.; Choi, W.; Chen, Z.; Suzuki, T.; Yoshioka, M.; Kawawaki, T.; Lee, D.; Negishi, Y. Elucidating Ligand Effects in Thiolate-Protected Metal Clusters Using $\mathrm{Au}_{24} \mathrm{Pt}(\mathrm{TBBT})_{18}$ as a Model Cluster. Nanoscale 2019, 11, 22089-22098. [CrossRef]

273. Kawawaki, T.; Negishi, Y.; Kawasaki, H. Photo/Electrocatalysis and Photosensitization Using Metal Nanoclusters for Green Energy and Medical Applications. Nanoscale Adv. 2020, 2, 17-36. [CrossRef]

274. Kawawaki, T.; Negishi, Y. Gold Nanoclusters as Electrocatalysts for Energy Conversion. Nanomaterials 2020, 10, 238. [CrossRef]

275. Hossain, S.; Imai, Y.; Motohashi, Y.; Chen, Z.; Suzuki, D.; Suzuki, T.; Kataoka, Y.; Hirata, M.; Ono, T.; Kurashige, W.; et al. Understanding and Designing One-Dimensional Assemblies of Ligand-Protected Metal Nanoclusters. Mater. Horiz. 2020, 7, 796-803. [CrossRef]

276. Negishi, Y.; Hashimoto, S.; Ebina, A.; Hamada, K.; Hossain, S.; Kawawaki, T. Atomic-Level Separation of Thiolate-Protected Metal Clusters. Nanoscale 2020, 12, 8017-8039. [CrossRef]

277. Kumar, B.; Kawawaki, T.; Shimizu, N.; Imai, Y.; Suzuki, D.; Hossain, S.; Nair, L.V.; Negishi, Y. Gold Nanoclusters as Electrocatalysts: Size, Ligands, Heteroatom Doping, and Charge Dependences. Nanoscale 2020, 12, 9969-9979. [CrossRef] [PubMed]

278. Ebina, A.; Hossain, S.; Horihata, H.; Ozaki, S.; Kato, S.; Kawawaki, T.; Negishi, Y. One-, Two-, and Three-Dimensional SelfAssembly of Atomically Precise Metal Nanoclusters. Nanomaterials 2020, 10, 1105. [CrossRef]

279. Hossain, S.; Suzuki, D.; Iwasa, T.; Kaneko, R.; Suzuki, T.; Miyajima, S.; Iwamatsu, Y.; Pollitt, S.; Kawawaki, T.; Barrabés, N.; et al. Determining and Controlling Cu-Substitution Sites in Thiolate-Protected Gold-Based 25-Atom Alloy Nanoclusters. J. Phys. Chem. C 2020, 124, 22304-22313. [CrossRef]

280. Kawawaki, T.; Imai, Y.; Suzuki, D.; Kato, S.; Kobayashi, I.; Suzuki, T.; Kaneko, R.; Hossain, S.; Negishi, Y. Atomically Precise Alloy Nanoclusters. Chem. Eur. J. 2020, 26, 16150-16193. [CrossRef]

281. Kawawaki, T.; Kataoka, Y.; Hirata, M.; Iwamatsu, Y.; Hossain, S.; Negishi, Y. Toward the Creation of High-Performance Heterogeneous Catalysts by Controlled Ligand Desorption from Atomically Precise Metal Nanoclusters. Nanoscale Horiz. 2021, 6 , 409-448. [PubMed]

282. Kawawaki, T.; Ebina, A.; Hosokawa, Y.; Ozaki, S.; Suzuki, D.; Hossain, S.; Negishi, Y. Thiolate-Protected Metal Nanoclusters: Recent Development in Synthesis, Understanding of Reaction, and Application in Energy and Environmental Field. Small 2021, 17, 2005328. [CrossRef]

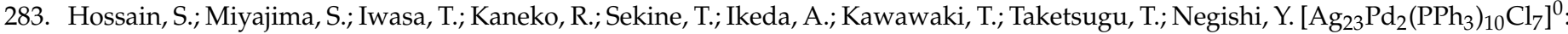
A New Family of Synthesizable Bi-Icosahedral Superatomic Molecules. J. Chem. Phys. 2021, 155, 024302. [CrossRef]

284. Lyu, H.; Hisatomi, T.; Goto, Y.; Yoshida, M.; Higashi, T.; Katayama, M.; Takata, T.; Minegishi, T.; Nishiyama, H.; Yamada, T.; et al. An Al-Doped $\mathrm{SrTiO}_{3}$ Photocatalyst Maintaining Sunlight-Driven Overall Water Splitting Activity for Over $1000 \mathrm{~h}$ of Constant Illumination. Chem. Sci. 2019, 10, 3196-3201. [CrossRef]

285. Ham, Y.; Hisatomi, T.; Goto, Y.; Moriya, Y.; Sakata, Y.; Yamakata, A.; Kubota, J.; Domen, K. Flux-Mediated Doping of SrTiO 3 Photocatalysts for Efficient Overall Water Splitting. J. Mater. Chem. A 2016, 4, 3027-3033. [CrossRef]

286. Qiu, B.; Du, M.; Ma, Y.; Zhu, Q.; Xing, M.; Zhang, J. Integration of Redox Cocatalysts for Artificial Photosynthesis. Energy Environ. Sci. 2021, 14, 5260-5288

287. Li, R.; Zhang, F.; Wang, D.; Yang, J.; Li, M.; Zhu, J.; Zhou, X.; Han, H.; Li, C. Spatial Separation of Photogenerated Electrons and Holes among $\{010\}$ and $\{110\}$ Crystal Facets of $\mathrm{BiVO}_{4}$. Nat. Commun. 2013,4,1432. [CrossRef]

288. Takata, T.; Jiang, J.; Sakata, Y.; Nakabayashi, M.; Shibata, N.; Nandal, V.; Seki, K.; Hisatomi, T.; Domen, K. Photocatalytic Water Splitting with a Quantum Efficiency of Almost Unity. Nature 2020, 581, 411-414. [CrossRef]

289. Khan, K.; Tao, X.; Zhao, Y.; Zeng, B.; Shi, M.; Ta, N.; Li, J.; Jin, X.; Li, R.; Li, C. Spatial Separation of Dual-Cocatalysts on One-Dimensional Semiconductors for Photocatalytic Hydrogen Production. J. Mater. Chem. A 2019, 7, 15607-15614. [CrossRef]

290. Khan, K.; Tao, X.; Shi, M.; Zeng, B.; Feng, Z.; Li, C.; Li, R. Visible-Light-Driven Photocatalytic Hydrogen Production on Cd $0.5 Z_{0.5} S$ Nanorods with an Apparent Quantum Efficiency Exceeding 80\%. Adv. Funct. Mater. 2020, 30, 2003731. [CrossRef]

291. Wang, D.; Hisatomi, T.; Takata, T.; Pan, C.; Katayama, M.; Kubota, J.; Domen, K. Core/Shell Photocatalyst with Spatially Separated Co-Catalysts for Efficient Reduction and Oxidation of Water. Angew. Chem. Int. Ed. 2013, 52, 11252-11256. [CrossRef] 
292. Zheng, D.; Cao, X.-N.; Wang, X. Precise Formation of a Hollow Carbon Nitride Structure with a Janus Surface to Promote Water Splitting by Photoredox Catalysis. Angew. Chem. Int. Ed. 2016, 55, 11512-11516. [CrossRef] [PubMed]

293. Zhao, F.; Zhang, N.; Li, H.; Zhang, X.; Luo, Z.; Wang, Y. Photocatalyst with Chloroplast-Like Structure for Enhancing Hydrogen Evolution Reaction. Energy Environ. Mater. 2021. [CrossRef]

294. Bai, S.; Wang, L.; Li, Z.; Xiong, Y. Facet-Engineered Surface and Interface Design of Photocatalytic Materials. Adv. Sci. 2017, 4, 1600216. [CrossRef]

295. Zhu, J.; Pang, S.; Dittrich, T.; Gao, Y.; Nie, W.; Cui, J.; Chen, R.; An, H.; Fan, F.; Li, C. Visualizing the Nano Cocatalyst Aligned Electric Fields on Single Photocatalyst Particles. Nano Lett. 2017, 17, 6735-6741. [CrossRef]

296. Chen, R.; Fan, F.; Dittrich, T.; Li, C. Imaging Photogenerated Charge Carriers on Surfaces and Interfaces of Photocatalysts with Surface Photovoltage Microscopy. Chem. Soc. Rev. 2018, 47, 8238-8262. [CrossRef] [PubMed]

297. Miyamoto, N.; Nakato, T. Liquid Crystalline Nanosheet Colloids with Controlled Particle Size Obtained by Exfoliating Single Crystal of Layered Niobate $\mathrm{K}_{4} \mathrm{Nb}_{6} \mathrm{O}_{17}$. J. Phys. Chem. B 2004, 108, 6152-6159. [CrossRef]

298. Teshima, K.; Niina, Y.; Yubuta, K.; Suzuki, T.; Ishizawa, N.; Shishido, T.; Oishi, S. Environmentally Friendly Growth of Layered $\mathrm{K}_{4} \mathrm{Nb}_{6} \mathrm{O}_{17}$ Crystals from a KCl Flux. Eur. J. Inorg. Chem. 2007, 2007, 4687-4692. [CrossRef]

299. Lee, S.; Teshima, K.; Niina, Y.; Suzuki, S.; Yubuta, K.; Shishido, T.; Endo, M.; Oishi, S. Highly Crystalline Niobium Oxide Converted from Flux-Grown $\mathrm{K}_{4} \mathrm{Nb}_{6} \mathrm{O}_{17}$ Crystals. CrystEngComm 2009, 11, 2326-2331. [CrossRef]

300. Maczka, M.; Ptak, M.; Majchrowski, A.; Hanuza, J. Raman and IR Spectra of $\mathrm{K}_{4} \mathrm{Nb}_{6} \mathrm{O}_{17}$ and $\mathrm{K}_{4} \mathrm{Nb}_{6} \mathrm{O}_{17} \cdot 3 \mathrm{H}_{2} \mathrm{O}$ Single Crystals. J. Raman Spectrosc. 2011, 42, 209-213. [CrossRef]

301. Kakimoto, K.-i.; Sugiyama, K.; Kagomiya, I. Direct Synthesis of Platelet $\mathrm{KNbO}_{3}$ Particles Using $\mathrm{KNb}_{3} \mathrm{O}_{8} \mathrm{Precursor}$ and $\mathrm{KNO}_{3}$ Self-Flux. J. Ceram. Soc. Jpn. 2010, 118, 696-700. [CrossRef]

302. Madaro, F.; Sæterli, R.; Tolchard, J.R.; Einarsrud, M.-A.; Holmestad, R.; Grande, T. Molten Salt Synthesis of $\mathrm{K}_{4} \mathrm{Nb}_{6} \mathrm{O}_{17}, \mathrm{~K}_{2} \mathrm{Nb}_{4} \mathrm{O}_{11}$ and $\mathrm{KNb}_{3} \mathrm{O}_{8}$ Crystals with Needle- or Plate-Like Morphology. CrystEngComm 2011, 13, 1304-1313. [CrossRef]

303. Rørvik, P.M.; Lyngdal, T.; Sæterli, R.; van Helvoort, A.T.J.; Holmestad, R.; Grande, T.; Einarsrud, M.-A. Influence of Volatile Chlorides on the Molten Salt Synthesis of Ternary Oxide Nanorods and Nanoparticles. Inorg. Chem. 2008, 47, $3173-3181$. [CrossRef]

304. Xu, C.-Y.; Wu, J.; Zhang, P.; Hu, S.-P.; Cui, J.-X.; Wang, Z.-Q.; Huang, Y.-D.; Zhen, L. Molten Salt Synthesis of Na $\mathrm{Ti}_{3} \mathrm{O}_{7}$ and $\mathrm{Na}_{2} \mathrm{Ti}_{6} \mathrm{O}_{13}$ One-Dimensional Nanostructures and Their Photocatalytic and Humidity Sensing Properties. CrystEngComm 2013, 15, 3448-3454. [CrossRef]

305. Yoshida, H.; Sato, M.; Fukuo, N.; Zhang, L.; Yoshida, T.; Yamamoto, Y.; Morikawa, T.; Kajino, T.; Sakano, M.; Sekito, T.; et al. Sodium Hexatitanate Photocatalysts Prepared by a Flux Method for Reduction of Carbon Dioxide with Water. Catal. Today 2018, 303, 296-304. [CrossRef]

306. Noureldine, D.; Anjum, D.H.; Takanabe, K. Flux-Assisted Synthesis of $\mathrm{SnNb}_{2} \mathrm{O}_{6}$ for Tuning Photocatalytic Properties. Phys. Chem. Chem. Phys. 2014, 16, 10762-10769. [CrossRef]

307. Niishiro, R.; Takano, Y.; Jia, Q.; Yamaguchi, M.; Iwase, A.; Kuang, Y.; Minegishi, T.; Yamada, T.; Domen, K.; Kudo, A. A $\mathrm{CoO}_{x}$-Modified $\mathrm{SnNb}_{2} \mathrm{O}_{6}$ Photoelectrode for Highly Efficient Oxygen Evolution from Water. Chem. Commun. 2017, 53, 629-632. [CrossRef]

308. Yamakata, A.; Ishibashi, T.-a.; Onishi, H. Kinetics of the Photocatalytic Water-Splitting Reaction on $\mathrm{TiO}_{2}$ and $\mathrm{Pt} / \mathrm{TiO}_{2} \mathrm{Studied}$ by Time-Resolved Infrared Absorption Spectroscopy. J. Mol. Catal. A Chem. 2003, 199, 85-94. [CrossRef]

309. Yamakata, A.; Kawaguchi, M.; Nishimura, N.; Minegishi, T.; Kubota, J.; Domen, K. Behavior and Energy States of Photogenerated Charge Carriers on Pt- or $\mathrm{CoO}_{x}$-Loaded $\mathrm{LaTiO}_{2} \mathrm{~N}$ Photocatalysts: Time-Resolved Visible to Mid-Infrared Absorption Study. J. Phys. Chem. C 2014, 118, 23897-23906. [CrossRef]

310. Yamakata, A.; Yeilin, H.; Kawaguchi, M.; Hisatomi, T.; Kubota, J.; Sakata, Y.; Domen, K. Morphology-Sensitive Trapping States of Photogenerated Charge Carriers on $\mathrm{SrTiO}_{3}$ Particles Studied by Time-Resolved Visible to Mid-IR Absorption Spectroscopy: The Effects of Molten Salt Flux Treatments. J. Photochem. Photobiol. A 2015, 313, 168-175. [CrossRef]

311. Yamakata, A.; Kawaguchi, M.; Murachi, R.; Okawa, M.; Kamiya, I. Dynamics of Photogenerated Charge Carriers on Ni- and Ta-Doped $\mathrm{SrTiO}_{3}$ Photocatalysts Studied by Time-Resolved Absorption and Emission Spectroscopy. J. Phys. Chem. C 2016, 120, 7997-8004. [CrossRef]

312. Vequizo, J.J.M.; Matsunaga, H.; Ishiku, T.; Kamimura, S.; Ohno, T.; Yamakata, A. Trapping-Induced Enhancement of Photocatalytic Activity on Brookite $\mathrm{TiO}_{2}$ Powders: Comparison with Anatase and Rutile $\mathrm{TiO}_{2}$ Powders. ACS Catal. 2017, 7, 2644-2651. [CrossRef]

313. Yamakata, A.; Vequizo, J.J.M. Curious Behaviors of Photogenerated Electrons and Holes at the Defects on Anatase, Rutile, and Brookite $\mathrm{TiO}_{2}$ Powders: A Review. J. Photochem. Photobiol. C 2019, 40, 234-243. [CrossRef]

314. Yabuta, M.; Takeda, A.; Sugimoto, T.; Watanabe, K.; Kudo, A.; Matsumoto, Y. Particle Size Dependence of Carrier Dynamics and Reactivity of Photocatalyst $\mathrm{BiVO}_{4}$ Probed with Single-Particle Transient Absorption Microscopy. J. Phys. Chem. C 2017, 121, 22060-22066. [CrossRef]

315. Takahata, R.; Yamazoe, S.; Maehara, Y.; Yamazaki, K.; Takano, S.; Kurashige, W.; Negishi, Y.; Gohara, K.; Tsukuda, T. Electron Microscopic Observation of an Icosahedral $\mathrm{Au}_{13}$ Core in $\mathrm{Au}_{25}(\mathrm{SePh})_{18}$ and Reversible Isomerization between Icosahedral and Face-Centered Cubic Cores in $\mathrm{Au}_{144}\left(\mathrm{SC}_{2} \mathrm{H}_{4} \mathrm{Ph}\right)_{60}$. J. Phys. Chem. C 2020, 124, 6907-6912. [CrossRef]

316. Arul, K.T.; Chang, H.-W.; Shiu, H.-W.; Dong, C.-L.; Pong, W.-F. A Review of Energy Materials Studied by in situ/Operando Synchrotron X-Ray Spectro-Microscopy. J. Phys. D Appl. Phys. 2021, 54, 343001. [CrossRef] 
317. Kowalski, P.M.; Meyer, B.; Marx, D. Composition, Structure, and Stability of the Rutile $\mathrm{TiO}_{2}(110)$ Surface: Oxygen Depletion, Hydroxylation, Hydrogen Migration, and Water Adsorption. Phys. Rev. B 2009, 79, 115410. [CrossRef]

318. Wang, H.-L.; Hu, Z.-P.; Li, H. Dissociation of Liquid Water on Defective Rutile $\mathrm{TiO}_{2}(110)$ Surfaces Using abinitio Molecular Dynamics Simulations. Front. Phys. 2018, 13, 138107. [CrossRef]

319. Zhou, G.; Liu, C.; Huang, L. Molecular Dynamics Simulation of First-Adsorbed Water Layer at Titanium Dioxide Surfaces. J. Chem. Eng. Data 2018, 63, 2420-2429. [CrossRef]

320. Valdés, Á.; Qu, Z.-W.; Kroes, G.-J.; Rossmeisl, J.; Nørskov, J.K. Oxidation and Photo-Oxidation of Water on TiO 2 Surface. J. Phys. Chem. C 2008, 112, 9872-9879. [CrossRef]

321. Chen, J.; Li, Y.-F.; Sit, P.; Selloni, A. Chemical Dynamics of the First Proton-Coupled Electron Transfer of Water Oxidation on TiO 2 Anatase. J. Am. Chem. Soc. 2013, 135, 18774-18777. [CrossRef]

322. Nurlaela, E.; Wang, H.; Shinagawa, T.; Flanagan, S.; Ould-Chikh, S.; Qureshi, M.; Mics, Z.; Sautet, P.; Le Bahers, T.; Cánovas, E.; et al. Enhanced Kinetics of Hole Transfer and Electrocatalysis during Photocatalytic Oxygen Evolution by Cocatalyst Tuning. ACS Catal. 2016, 6, 4117-4126. [CrossRef]

323. Li, B.; Wu, S.; Gao, X. Theoretical Calculation of a $\mathrm{TiO}_{2}$-based Photocatalyst in the Field of Water Splitting: A Review. Nanotechnol. Rev. 2020, 9, 1080-1103.

324. Hisatomi, T.; Domen, K. Reaction Systems for Solar Hydrogen Production via Water Splitting with Particulate Semiconductor Photocatalysts. Nat. Catal. 2019, 2, 387-399. [CrossRef]

325. Lin, L.; Hisatomi, T.; Chen, S.; Takata, T.; Domen, K. Visible-Light-Driven Photocatalytic Water Splitting: Recent Progress and Challenges. Trends Chem. 2020, 2, 813-824. [CrossRef] 